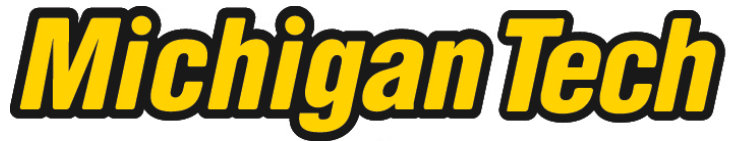 \\ Michigan Technological University Create the Future Digital Commons @ Michigan Tech
}

2015

\section{ELECTROCHEMICAL PROCESSES IN MICROFLUIDICS SYSTEMS UNDER AC ELECTRIC FIELDS}

Ran An

Michigan Technological University

Follow this and additional works at: https://digitalcommons.mtu.edu/etds

Part of the Chemical Engineering Commons, and the Electrical and Computer Engineering Commons Copyright 2015 Ran An

\section{Recommended Citation}

An, Ran, "ELECTROCHEMICAL PROCESSES IN MICROFLUIDICS SYSTEMS UNDER AC ELECTRIC FIELDS", Dissertation, Michigan Technological University, 2015.

https://doi.org/10.37099/mtu.dc.etds/951

Follow this and additional works at: https://digitalcommons.mtu.edu/etds

Part of the Chemical Engineering Commons, and the Electrical and Computer Engineering Commons 


\title{
ELECTROCHEMICAL PROCESSES IN MICROFLUIDICS SYSTEMS UNDER AC ELECTRIC FIELDS
}

\author{
By \\ Ran An
}

\begin{abstract}
A DISSERTATION
Submitted in partial fulfillment of the requirements for the degree of DOCTOR OF PHILOSOPHY

In Chemical Engineering

MICHIGAN TECHNOLOGICAL UNIVERSITY

2015
\end{abstract}

(C) Ran An 2015 
This dissertation has been approved in partial fulfillment of the requirements for the Degree of DOCTOR OF PHILOSOPHY in Chemical Engineering.

\section{Department of Chemical Engineering}

Dissertation Advisor: Adrienne. R. Minerick

Committee Member: $\quad$ Claudio Mazzoleni

Committee Member: Tony N. Rogers

Committee Member: Wen Zhou

Department Chair: S. Komar. Kawatra 
I dedicate this dissertation to my dear mother 


\section{Table of Contents}

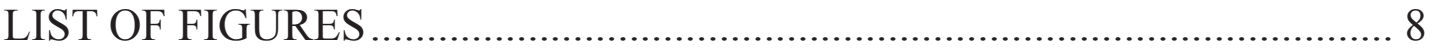

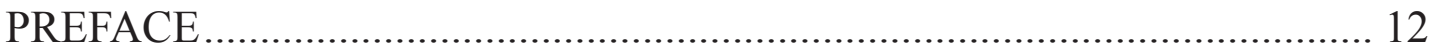

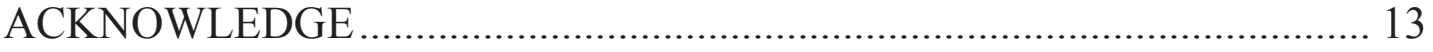

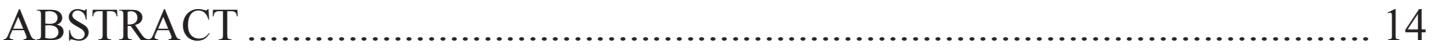

CHAPTER 1: INTRODUCTION AND OUTLINE ..................................... 16

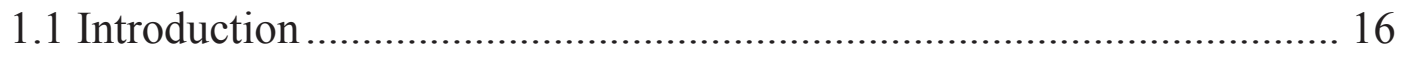

1.2 MEMS Technology and Microfluidics................................................ 18

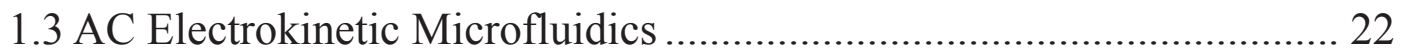

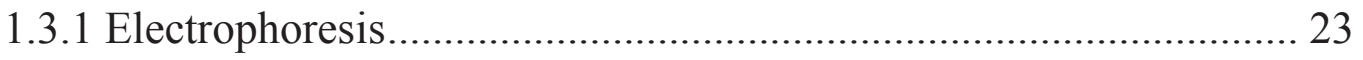

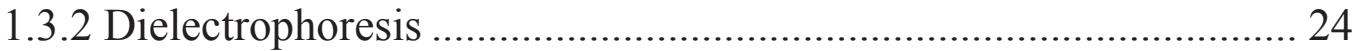

1.3.3 Alternating Current Electro-Osmosis (ACEO) …............................ 28

1.3.4 Alternating Current Electrothermal Flow (ACET) .......................... 30

CHAPTER 2 BACKGROUND AND REVIEW OF IONIC SOLUTION ELECTROCHEMISTRIES UNDER AC POTENTIAL ................................ 34

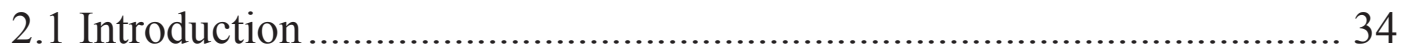

2.2 Non-Faradaic Processes ...................................................................... 35

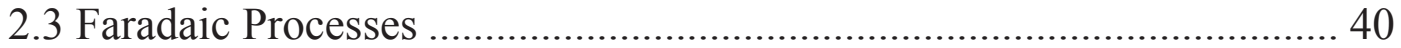

2.4 Faraday Processes under AC Electric Potential: Potential Drop and Experimental Work ............................................................................. 42

2.5 Non-Faraday Processes under AC Electric Potential: Double Layer and

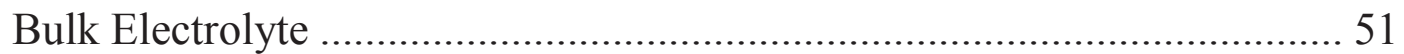

2.5.1 Non-Faraday Processes under AC Electric Potential within Double

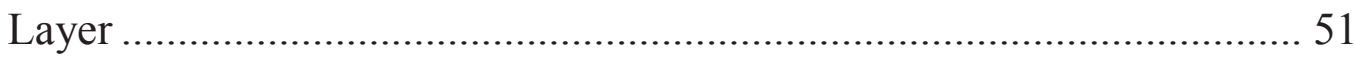

2.5.2 Non-Faraday Processes under AC Electric Potential in Bulk

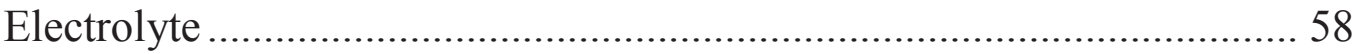

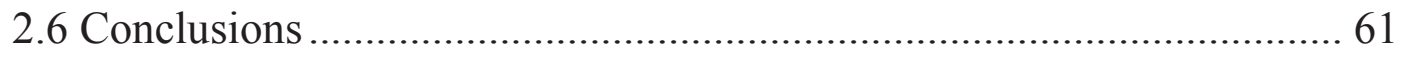

CHAPTER 3. SPATIALLY-VARIANT RED BLOOD CELL CRENATION IN 


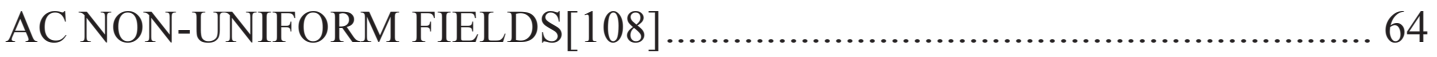

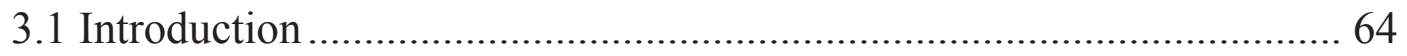

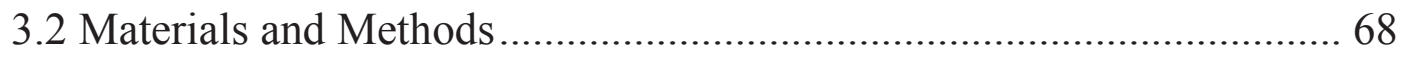

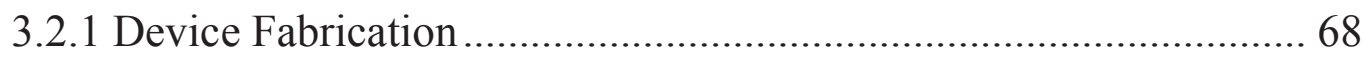

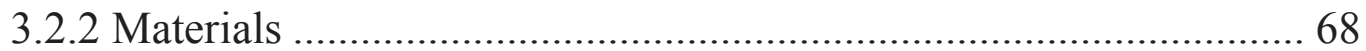

3.2.3 Red blood cell experiments.......................................................... 70

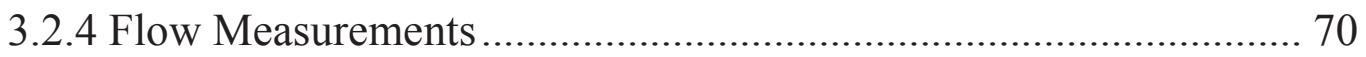

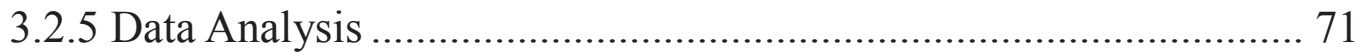

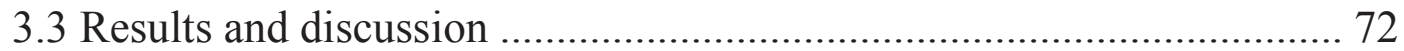

3.3.1 RBCs in Controlled Tonicity Media ............................................ 72

3.3.2 Time Dependence of RBC Crenation in Non-Uniform AC fields... 73

3.3.3 Peak-to-peak Potential and Frequency Dependence......................... 76

3.3.4 Quantification of Potentially Competing Mechanisms.................... 78

3.3.5 Impact from Electric Potential and Field ......................................... 78

3.3.6 Absolute Temperature Value and Change Temperature in Media.... 83

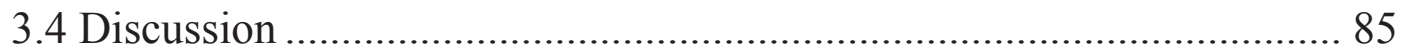

3.4.1 Physical Reason for Ion Concentration Gradient ........................... 86

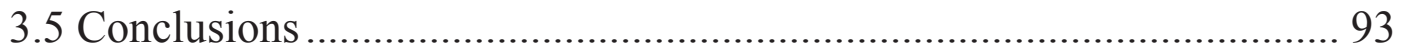

CHAPTER 4. SOLUTION PH CHANG IN NON-UNIFORM AC ELECTRIC FIELDS AT FREQUENCIES ABOVE THE ELECTRODE CHARGING

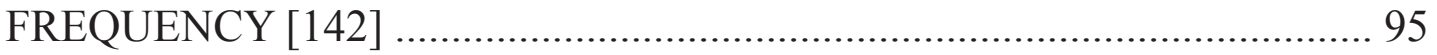

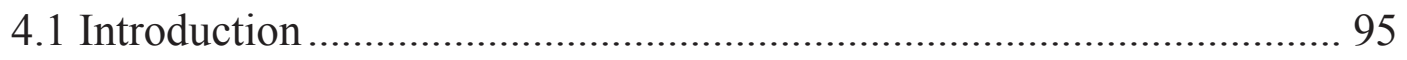

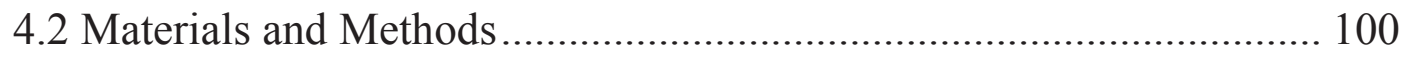

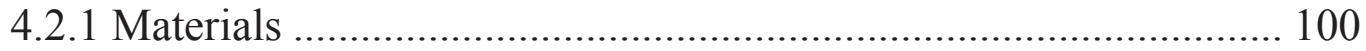

4.2.2 Microdevices Design and Fabrication .......................................... 100

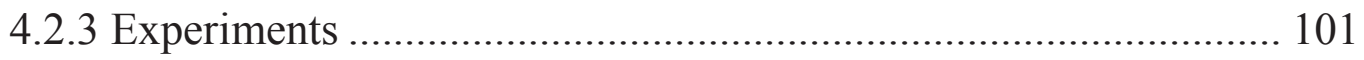

4.2.4 Data Analysis Methods ............................................................... 102

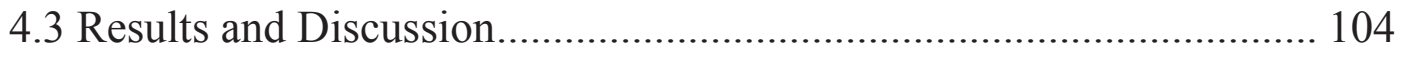

4.3.1 Fluorescein Calibrations ............................................................... 105

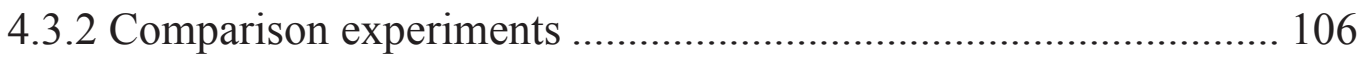


4.3.3 Time analysis of intensity change ................................................. 109

4.3.4 2-D Mapping of pH Change .........................................................113

4.3.5 Potential and Frequency Dependency............................................116

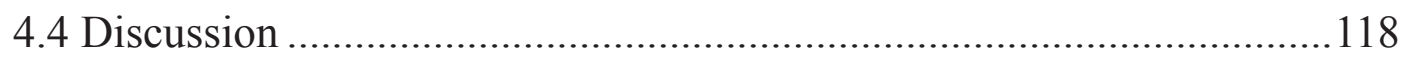

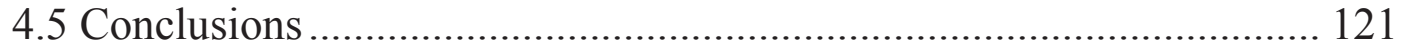

CHAPTER 5. STUDY OF ION CONCENTRATION CHANGE IN SPATIALLY NON-UNIFORM AC ELECTRIC FIELDS ........................... 123

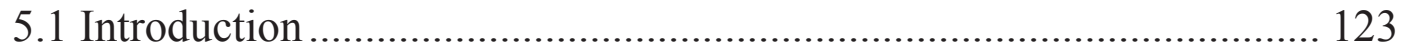

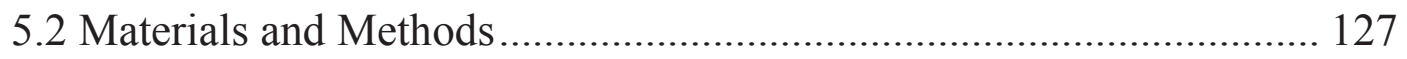

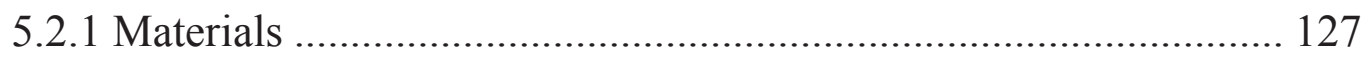

5.2.2 Microdevices Design and Fabrication ......................................... 128

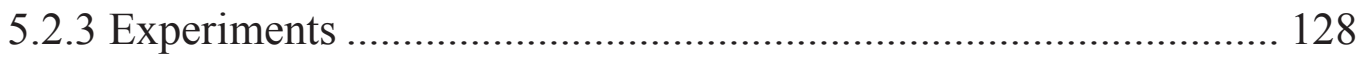

5.2.4 Data Analysis Methods .............................................................. 130

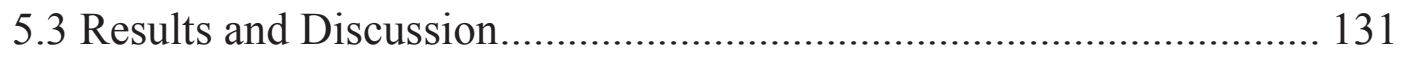

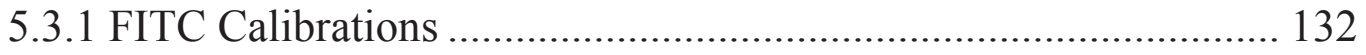

5.3.2 Control Experiments ................................................................ 133

5.3.3 Proposed Mechanism for the Established Ion Concentration Gradient 137

5.3.4 Spatial and Time analysis of Intensity Change ............................. 140

5.3.5 Peak-to-peak Potential and Frequency Dependency ...................... 148

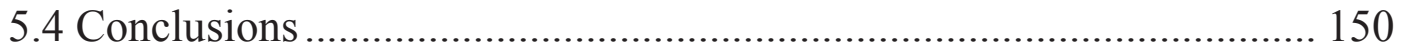

Chapter 6. CONCLUSIONS AND FUTURE WORKS ................................ 152

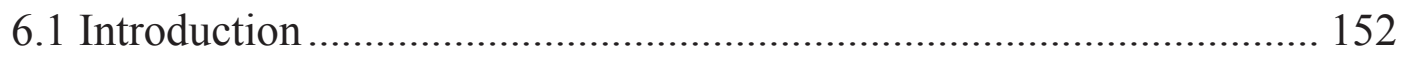

6.2 Biocompatibility Change in Spatially Non-uniform AC DEP Field.... 153

6.3 Detection of $\mathrm{pH}$ Change and $\mathrm{pH}$ Spatial Gradient in Spatially

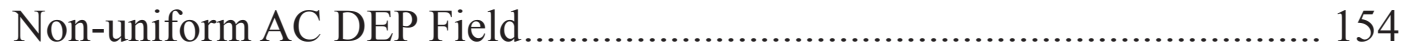

6.4 Detection of Ion Concentration Change and Ion Concentration Spatial Gradient in Spatially Non-uniform AC DEP Field .................................... 156

6.5 Contribution to the Discipline ............................................................. 160

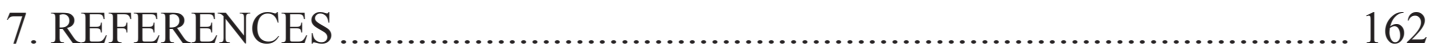


Appendix PROOFS OF PERMISSION TO REPRODUCE PUBLISHED

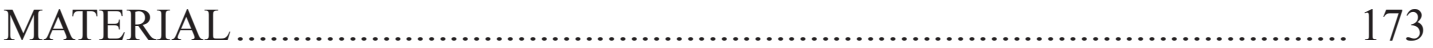




\section{LIST OF FIGURES}

Figure 1: Electrophoresis force on charged particle under electric field 23 Figure 2: Dielectric particles with neutral charges a) stayed uncharged in zero electric field, b) being polarized and experienced equal forces at both poles without net motion, c) being polarized in positive half of non-uniform AC electric field, experiencing a net force then net motion towards the area with higher electric field density and d): being polarized in negative half of non-uniform AC electric field, still experiencing a net force then net motion towards the area with higher electric field density

Figure 3: Schematic diagram showing fundamental principle of ACEO. The blue rectangular represents the glass slide, the yellow rectangular represent gold electrode. Black lines in circles are electric field lines 29

Figure 4: Schematic diagram showing flow pattern in ACEO system. .. 29 Figure 5: Simulated ACET flows: color plot shows temperature and arrows show flow velocity [46] (Figure reproduced with permission)

Figure 6: Diagram of electrical double layer configuration on a positively charged electrode. IHP and OHP stands for inner and outer Helmholtz plane, respectively 36

Figure 7: Simplified circuit for electrolyte between two electrolyte. Bulk electrolyte is considered as RC parallel circuit and electrical double layer is considered as RC series circuit on each side of electrodes 38

Figure 8: Process of electrochemical reaction: from bulk solution to electrical double layer to electrode surface

Figure 9: Output current efficiency dependence on input current at frequencies of 5-6, 21-22 and $64 \mathrm{~Hz}$ [62]. (Reproduced with permission)

Figure 10: Schematic diagram of impedance spectroscopy for interdigitated electrodes [80] (Reproduced with permission) ......... 53

Figure 11: Diagram of differential interferometric system designed for measurement of electrical double layer [82] (Reproduced with permission)

Figure 12: Weak frequency dependence of impedance to frequency [82] (Reproduced with permission) 
Figure 13: Comparison of electric potential $(\mathrm{c}, \mathrm{d})$ and ionic concentration $(e, f)$ distribution in (a) microchannel and (b) nanochannels [103] (Reproduced with permission) ……................................................ 61

Figure 14: Microdevice configuration. a): Photo of device, with fluid channel filled with green dye; b): Device design with dimensions shown, red boxes the working fluid chamber and c): Dimensions for red box in b), and red box is the area covered under 63x microscope. Units for all numbers are micrometers.

Figure 15: Red blood cells at $63 \mathrm{x}$ in a) Hypotonic $(0.72 \% \mathrm{NaCl})$ b) Isotonic $(0.9 \% \mathrm{NaCl})$ and c) Hypertonic $(7 \% \mathrm{NaCl})$ solutions d) Cell area distribution obtained from image analysis within the : Hypotonic: red; Isotonic: green; Hypertonic solution: blue. Cell deformation and crenation due to the hypertonic solution are observed and reflected in area analysis ......................................... 74

Figure 16: Time series of experiment at $500 \mathrm{kHz} 15 \mathrm{~V}$ peak-to-peak potential at $\mathrm{t}=0 \mathrm{~s}$ to $\mathrm{t}=580 \mathrm{~s}$ (a-d); histogram plot for cell area distribution at corresponding time point (e-h), and $\mathrm{i}$ is the cell average area change with time, with error bars shows standard deviation of cell sizes. Cells started to crenate in figure c) and crenation at higher level and higher amount in d). i) shows that cell area decrease with increase of time

Figure 17: Frequency and potential dependencya) frequency dependency of cell shrinkage percentage at $10 \mathrm{~V}$ (blue), $12.5 \mathrm{~V}$ (red), $15 \mathrm{~V}$ (green) and $17.5 \mathrm{~V}$ (purple); b): potential dependency of cell shrinkage percentage at $250 \mathrm{kHz}$ (red), $500 \mathrm{kHz}$ (blue), $750 \mathrm{kHz}$ (green) and $1 \mathrm{MHz}$ (purple). This shows cell deformation intensity increase with peak-to-peak potential and decrease with frequency, which implies the ion propagation increase with peak-to-peak potential and decrease with frequency. 77

Figure 18: Comparison experiments under 500 kHz, 15Vpp; First column are $\mathrm{t}=0,20$ and $580 \mathrm{~s}$ of experiments in uniform electric field; Second column are same time of experiments using medium solution contain $0.09 \% \mathrm{NaCl}$ and $4.5 \%$ dextrose. Third column are histograms of $\mathrm{t}=0,20$ and $580 \mathrm{~s}$ for nonuniform electric field, $0.9 \% \mathrm{NaCl}$; uniform electric field, $0.9 \% \mathrm{NaCl}$; and nonuniform electric field, $0.09 \% \mathrm{NaCl}$ and $4.5 \%$ dextrose, respectively. It can be observed that shift in distribution of cell area can be observed in c) while no shrinkage observed in f) and i) ...................................... 80

Figure 19: Cell Cell crenation explanation:crenation explanation: (a) 
Spatial variation of the electric field showing highest electric fields (red) near the vertical electrode. (b) Electrical field gradient showing the highest gradient at the vertical electrode. (c) COMSOL simulation of first 100 cycles of $15 \mathrm{Vpp}$ applied $500 \mathrm{kHz} \mathrm{AC}$ potential showing the oscillation of the ion concentration. Ion concentration increases slightly with each period and accumulates over time. 91

Figure 20: Microdevice designa): Photo of device, with fluid channel filled with green dye, red boxes the working fluidic chamber and $b$ ) Red boxed area under 10x microscope. Units for all numbers are micrometers 101

Figure 21: Dye properties study: a): Photobleaching property in water, b) Photobleaching property in methanol and c): $\mathrm{pH}$ calibrated to dye emission intensity. 104

Figure 22: Comparison experiments under $5 \mathrm{kHz}, 5.5 \mathrm{Vpp}$ :First column are $\mathrm{t}=0,8,120 \mathrm{~s}$ and intensity difference obtained by MATLAB image analysis of experiments under uniform electric field in methanol solvent; Second column are same time and image output of experiments under uniform electric field in water solvent. Third column are same information of experiments under non-uniform electric field in water solvent and fourth column are same information under non-uniform electric field in methanol solvent. It can be observed that intensity change can be observed in $n$ ) and o) while no change observed in $\mathrm{m}$ ) and $\mathrm{p}$ )....................................... 107

Figure 23: Intensity time analysis: Gray-scale 2-D dye intensity plot obtained from MATLAB image analysis at $\mathrm{t}=0,8,80$ and $120 \mathrm{~s}$ under $5.5 \mathrm{~V}_{\mathrm{pp}} 5 \mathrm{kHz}$; e)-h): 3-D MATLAB plot at same time point and i)-1): Calculated standard deviation of 5 repeats shown in the first two rows. Red line in figure $\mathrm{m}$ ) shows the position of sampled line intensity. Figure $n$ ) is showing the time dependency of the average of sampled line intensity.

Figure 24: $\mathrm{pH}$ change time analysis:a) and b): $\mathrm{pH}$ change, c) and d): absolute $\mathrm{pH}$ value, e) and $\mathrm{d}$ ): $\mathrm{pH}$ gradient at time $=60 \mathrm{~s}$ and $120 \mathrm{~s}$, respectively. Figure g) shows time dependence of $\mathrm{pH}$ change (black line) and absolute $\mathrm{pH}$ value (red line) 114

Figure 25: a): potential dependency of $\mathrm{pH}$ change (black) and absolute $\mathrm{pH}$ value (red) at $5 \mathrm{kHz}$ and $\mathrm{b}$ ): frequency dependency of $\mathrm{pH}$ change (black) and absolute $\mathrm{pH}$ value (red) at $5.5 \mathrm{Vpp}$. a): potential dependency of $\mathrm{pH}$ change (black) and absolute $\mathrm{pH}$ value (red) at 5 
$\mathrm{kHz}$ and $\mathrm{b}$ ): frequency dependency of $\mathrm{pH}$ change (black) and absolute $\mathrm{pH}$ value (red) at $5.5 \mathrm{~V}_{\mathrm{pp}}$. This shows $\mathrm{pH}$ change increase with peak-to-peak potential and decrease with frequency, which implies the Faradaic reaction increase with peak-to-peak potential and decrease with frequency.

Figure 26: Microdevice design. a): Photo of device, with fluid channel filled with green dye, red boxes the working fluidic chamber and $b$ ): Red box area under 10x microscope, c): Side view of the device. Units for d): COMSOL simulation of the gradient of electric field in labeled area in Fig. 26b. All numbers are micrometers.

Figure 27: Dye properties study a): Photobleaching property for FITC; b) Photobleaching property in for $\mathrm{Rb}$; c) FITC emission intensity calibrated with FITC concentration and d) Rb emission intensity calibrated with $\mathrm{Rb}$ concentration 131

Figure 28: Comparison experiments under $100 \mathrm{~Hz}, 10 \mathrm{Vpp}$; First column are $\mathrm{t}=0,7,60 \mathrm{~s}$ and FITC intensity difference obtained by MATLAB image analysis of experiments under uniform electric field; Second column are same time and image output of $\mathrm{Rb}$ intensity; Third column are same time and image output of experiments under nonuniform electric field for ionized FITC; Fourth column are same information of experiments using non-ionized $\mathrm{Rb}$ dye in non-uniform electric field It can be observed that intensity increase can be observed in o) while no change observed m) n) and p).... 135

Figure 29: Comparison between contour plot of experimental emission intensity at $\mathrm{t}=7$ and $30 \mathrm{~s}(\mathrm{a}, \mathrm{b})$ and COMSOL simulated electric field density represented at different color scales $(\mathrm{c}, \mathrm{d})$........................ 139

Figure 30: Ion concentration time analysis: a)-d): Gray-scale 2-D dye intensity plot obtained from MATLAB image analysis at $\mathrm{t}=0,7,30$ and 60s under 20Vpp $100 \mathrm{~Hz}$; e)-h): 3-D MATLAB plot at same time point and i)-1): Matlab contour plot showing the dye intensity gradient. Yellow box in figure $\mathrm{m}$ ) shows the position of sampled intensity. Figure n) is showing the time dependency of the average of sampled intensity. 142

Figure 31: a) : potential dependency of intensity change at $100 \mathrm{~Hz}$ and b): frequency dependency of intensity at $10 \mathrm{Vpp}$. This shows intensity change increase with peak-to-peak potential and decrease with frequency, which implies the ion concentration gradient increase with peak-to-peak potential and decrease with frequency. ........... 148 


\section{PREFACE}

Chapter 3, and 4 of this dissertation have previously been published in academic journals, and are reproduced with the permission of their respective publishers. Chapter 5 will be published in academic journal and the permission for reproduction will be requested once it is published. I was and will be the first author of all three articles. Also, I have done most of the experimental work and data analysis work presented in this dissertation. Part of data analysis in chapter 3 and experimental work in chapter 4 have been completed by undergraduate student Katherine Massa. All chapters are written under direction of my adviser Dr. Adrienne Minerick and her collaborator Dr. David Wipf from Mississippi State University.

Proofs of permission from the publishers of the reproduced articles in chapter 3 and 4 can be found in the appendix. 


\section{ACKNOWLEDGE}

First and foremost, I thank my parents Hongxia and Aiguo. They have given me endless unconditional love and support through all my 5 years phd study. I would not have been able to finish this work without their support.

Dr. Adrienne Minerick and my colleagues in MD-ERL research group. Dr. Minerick has given me the precious opportunity to work with this challenging and very interesting project and also offered me guidance as a friend, mentor and advisor. My colleagues, Kaela, Aytug, CJ, Tayloria, Hector, Hwiyong and Zhichao are great people to work with and we are all good friends to each other.

My committee members, Dr. Claudio Mazzoleni, Dr. Tony Rogers and Dr. Wen Zhou have also offered me significant guidance and help. I very much appreciate all their patience and mentoring. Also I would like to than to Dr. Paul Bergstrom, William Knudsen and Nupur, who have offered me great help in microfabrication processes. Finally, I appreciate to NSF, Department of Chemical Engineering in Michigan Technological University and Dr. Adrienne Minerick for funding my research and graduate studies through research grants and teaching assistant position. 


\section{ABSTRACT}

Alternating current (AC) electric signal has been widely applied in microfluidic systems to induce $\mathrm{AC}$ electrokinetic behavior. AC electrokinetic phenomena including $\mathrm{AC}$ electrophoresis, $\mathrm{AC}$ dielectrophoresis, $\mathrm{AC}$ electroosmosis flow and $\mathrm{AC}$ electrothermal flow are widely applied in (bio)particle sorting, separation and concentration and micropumps. However, numbers of non-ideal AC electrokinetic behaviors have been reported: human erythrocyte deformation in $\mathrm{AC}$ dielectrophoresis system has been observed; flow reversal in $\mathrm{AC}$ electroosmosis flow pumps has also been reported. In this dissertation, a systematic study on human erythrocyte crenation in AC dielectrophoresis system was firstly conducted. Multiple possible physical mechanisms inducing cell crenation including temperature, temperature jump, $\mathrm{pH}$, shear force and osmotic pressure was examined. $\mathrm{pH}$ change and osmotic pressure change induced by ionic concentration change was attributed as mechanisms induced cell crenation. Such $\mathrm{pH}$ change and ionic concentration gradient in $\mathrm{AC}$ non-uniform electric fields were then further studied. $\mathrm{pH}$ change was detected using Fluorescein sodium salt in sodium chloride solution at relative frequency $\sim 5$ to 25 times to electrode charging frequency. Time, spatial, frequency and peak-to-peak potential dependencies have been examined on such $\mathrm{pH}$ change and $\mathrm{pH}$ gradient. Ion concentration behavior was detected by using ionized FITC molecule as fluorescing ion dissolved in inert solvent methanol. Electrode surface is also coated with $\mathrm{HfO}_{2}$ dielectric layer to minimize effect from electrochemical reaction on FITC emission intensity. Time, spatial, frequency and peak-to-peak potential dependencies have also been examined on ion concentration change and ion concentration gradient. 
Both $\mathrm{pH}$ change and ion concentration change have been observed under high relative $\mathrm{AC}$ frequency. 


\section{CHAPTER 1: INTRODUCTION AND OUTLINE}

\subsection{Introduction}

Microelectromecahnical system (MEMS) is a technology based on small scale devices at micrometer scales[1-3]. Microfluidics, also referred to as "microdevices" or "lab-on-a-chip" devices, is generally an emerging technology over the past 40 years with the development of material and fabrication technologies. Similar to traditional MEMS, the fabrication of microfluidics systems following standard procedures have been developed from the electronics industry including patterning, photolithography, etching and sputtering etc. The significance of traditional MEMS is that such technology allows highly integrated circuit to perform large scale automation and computation to replace macroscopic circuits consisting massive components in the early 1990s $[4,5]$. The microfluidics systems have potential to miniaturize the automation and integration of biological sample treatments which currently require large scale robotic workstations and require large amount of time, labor and expenses[6].

The research presented in this dissertation was initiated by an encountered non-ideal cell deformation in microdevices under $\mathrm{AC}$ electric fields. Multiple possible physical mechanisms are examined on such cell deformation in the first part of this dissertation and is concluded to be induced by solution biocompatibility change in microdevices driven by $\mathrm{AC}$ electric fields. General electrolyte electrochemical reactions and mass transport are then studied to understand and propose solution to such fluid biocompatibility change problem. In the second part of this dissertation, solution property 
change induced by Faradaic reaction under AC electric potential is studied. While a number of reactions are potentially possible, $\mathrm{pH}$ change induced by water electrolysis was reported to be the major concern in microfluidics systems $[7,8]$ thus $\mathrm{pH}$ behavior is examined using $\mathrm{pH}$ sensitive fluorescent dye with respect to time and spatial position under $\mathrm{AC}$ fields in this dissertation. In the third part of this dissertation, effects from electromigration are examined using ionized fluorescing molecules. Effects from electrochemical reaction are minimized by using inertia organic solvent and coating conducting metal electrode with a dielectric layer. Potentially induced flow is also examined and the effect from diffusion is also considered and discussed.

The results from the research contribute to the field of electrokinetic microdevices technology by revealing solution properties changes under spatially non-uniform electric field. Previous studies only examined electrochemical reactions by detecting solution $\mathrm{pH}$ change under uniform electric field under low frequencies while in this dissertation, solution $\mathrm{pH}$ change was detected in non-uniform electric fields under higher frequencies, which are more relevant to real experimental conditions in electrokinetic microdevices. Also, the general presumption of uniform electrolyte concentration is challenged by results obtained from the examination of electromigration effect showing significant ion concentration gradient existence in AC electric field driven microdevices. Non-ideal behaviors such as flow reversal and solution biocompatibility change can be explained according to those results. Also, the results can facilitate the design of experiments to avoid solution properties changes by selectively choosing 
microdevice experimental condition such as solution initial concentration, conductivity, operational frequencies and potentials.

The following sections in this chapter discuss general concept of microdevices, $\mathrm{AC}$ electrokinetically driven microdevices and the motivation for the research. This introduction chapter also concludes with an outline of the remainder of this dissertation.

\subsection{MEMS Technology and Microfluidics}

As discussed previously, microdevices can be regarded as a specific branch of MEMS based on fluidics [1,9, 10]. A brief history of MEMS is first introduced as following[2]: in 1500, the very early lithographic process was developed to enable the etching of sub-mm features required by micromachining, which are the processes that deposits, etches or defines materials with features in micrometer scale or even smaller. However, not until the development of Ge and Si based pure semiconductors in 1940s have initiated the derivation process from micromachining processes into integrated circuits (ICs) $[2,11]$. While the point-contact transistor was invented and semiconductor circuit industry started in 1947 then the performance of semiconductor transistors also improved in 1949, the cost and reliability was still not sufficient. In 1960, planar batch-fabrication process was invented. Such process decreased the cost of semiconductor devices while significantly improved their reliability. What's more, such technique enabled process to integrate multiple devices into one piece. At the same year, the IC industry started the evolution towards miniaturization and increasing complexity because of the invention of metal-oxide-semiconductor field-effect transistor 
(MOSFET). In 1964, Nathenson created the first engineered batch-fabricated MEMS devices designed as resonant gate transistor. In 1970, the demands for integrated circuits significantly increased due to the development of microprocessor techniques. In 1970s and 1980s, the demands came from automotive industry also facilitated the growth of MEMS and several companies started to commercialize the MEMS production. In 1984, Howe and Muller [5] produced the first polysilicon surface micromachined MEMS device integrated with circuits and made the fundament form current MEMS products. In 1991, MEMS machining was extended from 2 dimensional to 3 dimensional by extending the surface micromachined polysilicon process accomplished by Pister et al. Finally, from 1990s to today, the number of MEMS devices, technologies and applications significantly increased and expanded to multiple areas.

There are three major motivations for the development of microfluidics system: A. to generate new version of more versatile and compacted microscale chemistry and biochemistry techniques such as gas chromatography, high-pressure liquid chromatogramphy and capillary electrophoresis; B. to develop detectors for chemical and biological threats to counter the chemical and biological weapons from military and terrorist threats; and C. to develop bio-analytical methods that offer high sensitivity and resolution required by the explosion of genomics.

Combining the developed MEMS system with the above needs, a miniaturized gas-chromatography was developed in 1970s as the first microfluidic device [12]. After that, four pioneering laboratories (Manz [13], 
Harrison [14], Ramsey [15] and Mathies [16]) initiated the research on performing analysis in aqueous phase using microfluidic systems.

The application of poly(dimethylsiloxane) (PDMS) also significantly facilitated the development of microfluidic system. PDMS is a polymer material shows promising properties as: 1) micron scale features can be generated in PDMS on a reusable mask such as silicon wafer; 2) PDMS has high optical transparency down to ensure the proper optical detection under microscope objectives; 3) the curing process for elastomer is simple and is taken under low temperature down to 65 Celsius; 4) PDMS is a non-toxic material offering good biocompatibility to mammalian cells; and 5) PDMS can be sealed on other materials such as glass slide using covalent bonds generated by air plasma [10] [17] [18]. According to those distinct properties, the majority of microfluidic systems for biological and water-based research was developed based on PDMS.

The fundamental needs for fabricating microfluidic system now can be fulfilled by the combination of techniques from microelectromechanics and the development of PDMS. The fabrication procedure then includes patterning over the substrates layer, which functions as MEMS and PDMS layer functions as the microfluidic channel and chamber.

For the micro-substrates under microfluidic layer [19], standard soft lithography methods is widely used due to its low cost and less complicated process. By using the film mask, photoresist and lithography process, desired pattern with photoresist can be fabricated on substrates such as silicon wafer 
and glass slide. Then, metal or dielectric material can be deposited using sputtering or e-beam deposition. Substrates with desired metal or dielectric pattern then can be obtained after removing the photoresist using photoresist remover or organic solvents. Mold for fabrication of PDMS layer can be obtained by similar lithography process on masks such as silicon wafer. Elastomer is then cured on the mold and desired pattern can be obtained. Finally, PDMS is irreversibly sealed on the substrate with proper alignment using air generated plasma and microfluidic device with desired pattern is then obtained.

Research using microfluidic device has been widely conducted in numerous areas [20]. Genomics and proteomics analytical systems were developed for performing rapid genomic and DNA tests. Chemical and biological defense test devices were developed for detecting toxins or biological attacks. Environmental test devices can be used to perform in situ examination on water contaminations. Purification of biological samples such as Polymerase Chain Reaction (PCR) was also developed in microfluidics and are currently booming in industrial applications ( $90 \%$ of microfluidics related job opportunities currently are related to PCR processing). Micropump was also developed using different electrokinetic mechanisms to induce laminar flow and pump fluids in microfluidics. Another major application is clinical biomicrofluidics. Cell studies such as cell separation, concentration, and sorting were conducted in various designs of microfluidics; point of care diagnostic devices applied in immunological studies were also developed; microfluidics designed for bodily fluids handling such as blood plasma was also realized. 
Microfluidics can be driven by multiple forces such as traditional shear force or stress in pressure driven flow, meniscus forces in systems with droplets or air bubbles and currently widely used alternating current (AC) driven electrokinetic forces, which will be the focus in this dissertation.

\subsection{AC Electrokinetic Microfluidics}

Electrokinetic phenomena includes multiple physical effects that occur on fluids containing particles which can be solid, liquid or gas (i.e., plasma) with size at micro- or nanometer scale. Lyklema [21] reported that the electrokinetic phenomena includes: 1) electrophoresis, which describes particle motion induced by the charge on particle and electric field; 2) electro-osmosis, which describes the motion of liquids induced by electric field; 3) diffusiophoresis, which describes the motion of particles induced by chemical potential gradient generated by concentration gradient; 4) capillary osmosis, which describes the motion of fluids in porous body induced by chemical potential gradient generated by concentration gradient; 5) sedimentation potential, describes the electric potential generated by the sedimentation process of colloidal (solid) particles; 6) streaming potential, describes the electric potential generated by the movement of fluids; 7) colloidal vibration current, which describes electric current generated by the vibration of colloidal (solid) particles induced by ultrasound; 8) electric sonic amplitude, which describes the ultrasound generated by the colloidal (solid) particles responding to the applied AC electric potential; and 9) dielectrophoresis, which describes a dielectric (electrically neutral) particle responding to the electric field due to the effect of induced dipole. 
$\mathrm{AC}$ electrokinetic is the electrokinetic phenomena induced by alternating current potential and is one of the major driving forces for microfluidics systems. The four major AC electrokinetic behaviors related to this dissertation, Electrophoresis, Dielectrophoresis, AC electroosmosis and AC electrothermal flow will be introduced in the following sections [22].

\subsubsection{Electrophoresis}

Electrophoresis is a term describing particle motion induced by the non-zero charge on target particle responding to electric field [23]. The interaction between charges on the particle and electric field introduces Coulomb force as shown in Fig. 1, where $\vec{E}$ is electric field, $\mathrm{q}$ is particle charge and $\vec{F}$ is the Coulomb force.

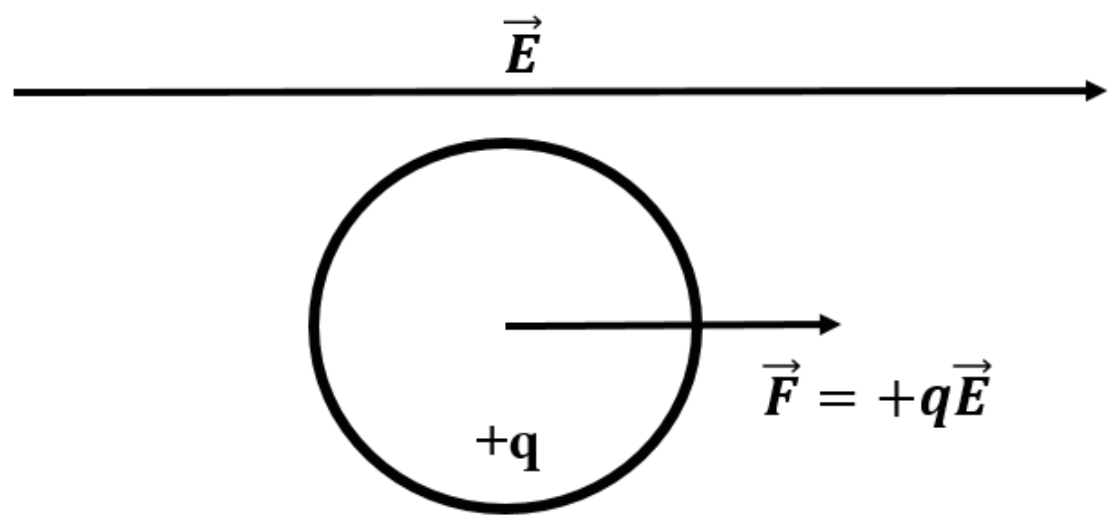

Figure 1: Electrophoresis force on charged particle under electric field

the total Coulomb force on the particle thus is given by the integration of product of charge and electric field: 


$$
\vec{F}_{E P}=Q \vec{E}=\int_{S} \sigma_{q} d S \vec{E}
$$

Where, $\mathrm{Q}$ is the total charge on particle, equals to the surface integration of surface charge density $\sigma_{q}$. Many biological samples such as DNA or cells have non-zero surface charge that will have electrophoresis effects under electric field. Electrolyte ions also suffer the electrophoresis effects causing solution property to change even under the non-polarizable electrode condition (i.e., zero Faradaic reaction rate). Under AC cases, the general assumptions of zero time averaged movement of particle or ion was normally made due to the oscillating nature of electric field and the solution condition thus was assumed to be constant [24]. However, in the following sections of this dissertation, this assumption was challenged and proved to be invalid in special cases.

\subsubsection{Dielectrophoresis}

Dielectrophoresis is a phenomena induced by the motion introduced by the interaction of electric fields and dielectric particle carrying zero net charge [25]. Without the impact from electric field, as shown in Fig. 2(a), the particle remains electrically neutral in electrolyte solution so zero motion is introduced. Once external uniform electric field is applied, as shown in Fig. 2(b), equal amount of positive and negative charges in the dielectric particle moves and accumulated at either side of the particle. The amount of accumulated charge

depends on the strength of electric field, conductivity (describing the ability of free charge, i.e. ionized electrolyte, move inside the liquid solvent), permittivity (describing the ability of charge accumulation and ability of electric field passing through) for both the electrolyte and the dielectric 
particle. In general, the capability for a material to produce charges in response to the electric field is described by the term polarizability.

a)

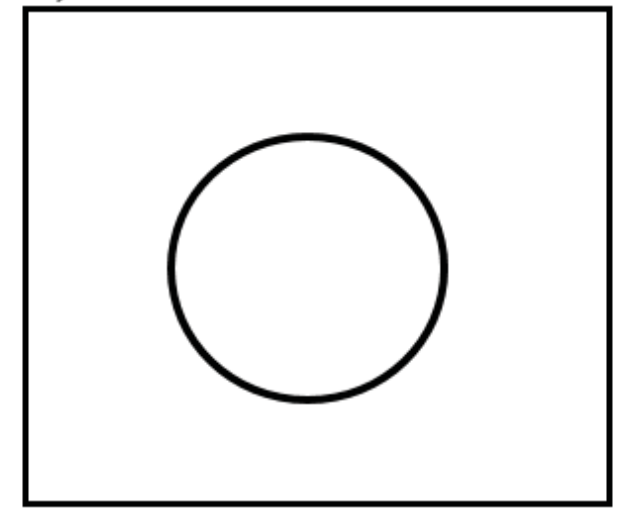

c)

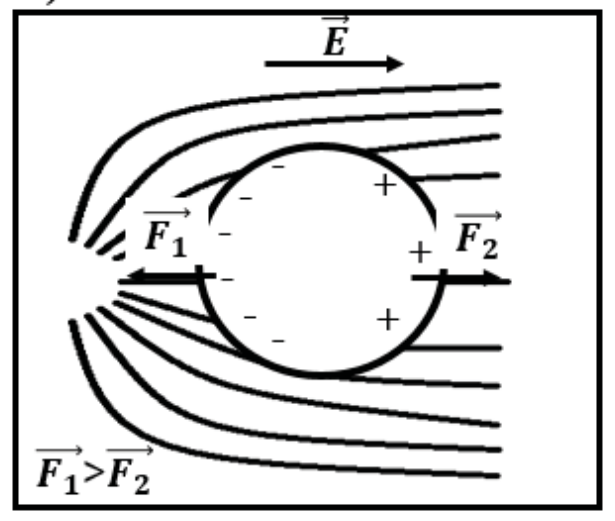

b)

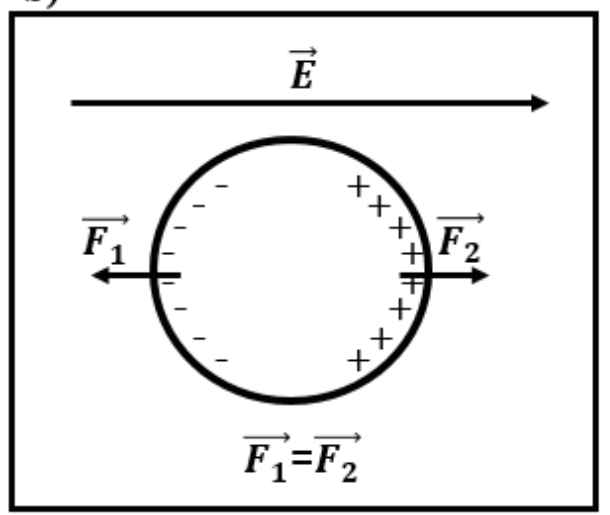

d)

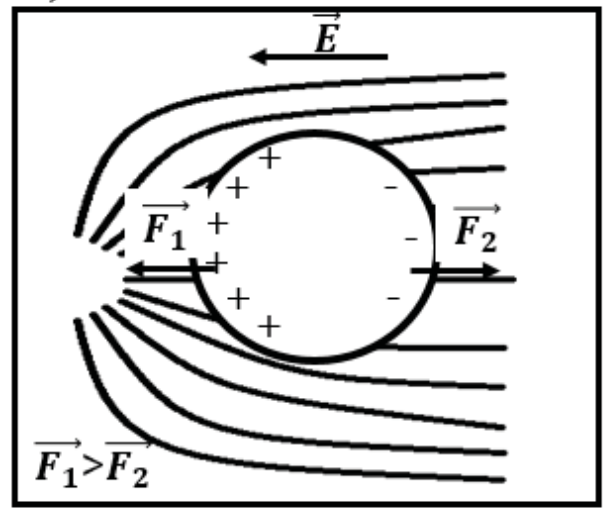

Figure 2: Dielectric particles with neutral charges a) stayed uncharged in zero electric field, b) being polarized and experienced equal forces at both poles without net motion, c) being polarized in positive half of non-uniform $\mathrm{AC}$ electric field, experiencing a net force then net motion towards the area with

higher electric field density and d): being polarized in negative half of non-uniform AC electric field, still experiencing a net force then net motion towards the area with higher electric field density

Under the condition of uniform electric field, the induced positive and negative charges on both poles of the particle stay in equal amount and the electric field strength are also equal which leads to two forces with equal 
magnitude but in exact opposite direction. As a result, the net force on the particle is still zero and no particle motion can be induced under the application of such uniform electric field.

A non-uniform electric field design then is necessary to induce motion on dielectric particle. As shown in Fig. 2(c), non-uniform electric field can induce unequal polarization effect. Greater amount of charges are induced in the higher field density pole and less amount of charges are induced in the lower field density pole. As a result, the induced force at high field density pole is the opposite to the force induced at low field density pole but with a higher magnitude. In Fig. 2(c) where the electric field direction is from left to right, negative charges is induced in the high field density pole thus the net force direction is toward the left side.

Under AC condition, the field is oscillating with time. Fig. 2(d) shows the other half cycle in the same AC period showing in Fig. 2(c). In this case, the direction of electric field is opposite which passes from right to the left. However, the signs of induced charges on each pole of the particle are also opposite to the first half shown in Fig. 2(c). As a result, the net dielectric force is still directing to the higher field density pole, which would introduce the motion in the exact same direction and magnitude as in the first half period in Fig. 2(c).

There are established theories illustrating that time averaged dielectrophoresis in AC electric field that can be written as [25]: 


$$
\left\langle\overrightarrow{F_{D E P}}>=\frac{1}{2} v \operatorname{Re}[\tilde{\alpha}] \nabla|\vec{E}|^{2}\right.
$$

for the special case of spherical particle, the time averaged DEP force can be written as:

$$
<\overrightarrow{F_{D E P}}>=\pi \varepsilon_{m} a^{3} \operatorname{Re}\left[f_{C M}\right] \nabla|\vec{E}|^{2}
$$

where, $\varepsilon_{m}$ is permittivity of medium solution, $a$ is radius of the particle and $f_{C M}$ is the Clausius-Mossotti factor describing a relaxation in the effective permittivity or polarisability of a particle as:

$$
f_{C M}=\frac{\tilde{\varepsilon}_{p}-\tilde{\varepsilon}_{m}}{\tilde{\varepsilon}_{p}+2 \tilde{\varepsilon}_{m}}
$$

where $\tilde{\varepsilon}_{p}$ and $\tilde{\varepsilon}_{m}$ are complex permittivity of particle and medium solution.

The real part of the Clausius-Mossotti factor then determines the direction and magnitude of DEP force at each frequency: Positive DEP is induced if the polarisability of particle is greater than medium solution $\tilde{\varepsilon}_{p}>$ $\tilde{\varepsilon}_{m}$, where $\operatorname{Re}\left[f_{C M}\right]>1$, resulting in the direction of particle motion pointing from lower to higher electric field density area. Negative DEP is induced if the polarisability of particle is smaller than medium solution $\tilde{\varepsilon}_{p}<$ $\tilde{\varepsilon}_{m}$, where $\operatorname{Re}\left[f_{C M}\right]<1$, resulting in the direction of particle motion pointing from higher to lower electric field density area.

The DEP phenomena is currently widely applied into (bio)particle sorting [26-31], concentrating [32] and separation [33]. Cancer cells [34], red blood cells [32], stem cells [Tayloria] even micro or nanoparticles [35] have been reported to be responding to DEP forces. Some of the DEP techniques 
have also been turned into commercialized applications such as blood typing and rare cell enrichment.

\subsubsection{Alternating Current Electro-Osmosis (ACEO)}

ACEO was firstly observed as an unexpected phenomena from DEP experiments [22]. In 1999, Ramos et al. [36] firstly reported such phenomena as the interaction between non-uniform electric field and the induced charge in the diffuse double layer. The fundamental principle is shown in Fig. 3: AC electric signal is applied across the two planar electrodes. In one half cycle of the signal, positive potential is applied on the left electrode while negative potential is applied on the right electrode. Due to the effect of electrode polarization (ions being attracted to electrode by electrostatic forces), electrical double layer with anions and cations are established on left and right electrodes, respectively. The ions in diffuse layer then are affected by the tangential part of the electric field and a net motion of ions is generated and such ion motion is also bringing the solvent (normally water) molecules surrounding the ion to move due to the shear stress and thus inducing the fluid motion. The direction of such motion on the left electrode is pointing to the left and on the right electrode pointing to right. In the other half cycle of the signal, opposite potential is applied on each electrodes thus ions with opposite signs are also induced resulting in the same force direction. As a result, as shown in Fig.4 such constant force leads to a non-zero time-averaged force on the fluid and a steady-state flow is induced with a time averaged fluid velocity $u_{x}$ as [36]:

$$
<u_{x}>=\frac{1}{2} \operatorname{Re}\left[\frac{\sigma_{q o} E_{t}^{*}}{\eta \kappa}\right]=\frac{1}{8} \frac{\varepsilon \emptyset_{0}^{2} \Omega^{2}}{\eta x\left(1+\Omega^{2}\right)^{2}}
$$


where,

$$
\Omega=\frac{1}{2} \pi \kappa x \frac{\varepsilon}{\sigma} \omega
$$

is the non-dimensional frequency.

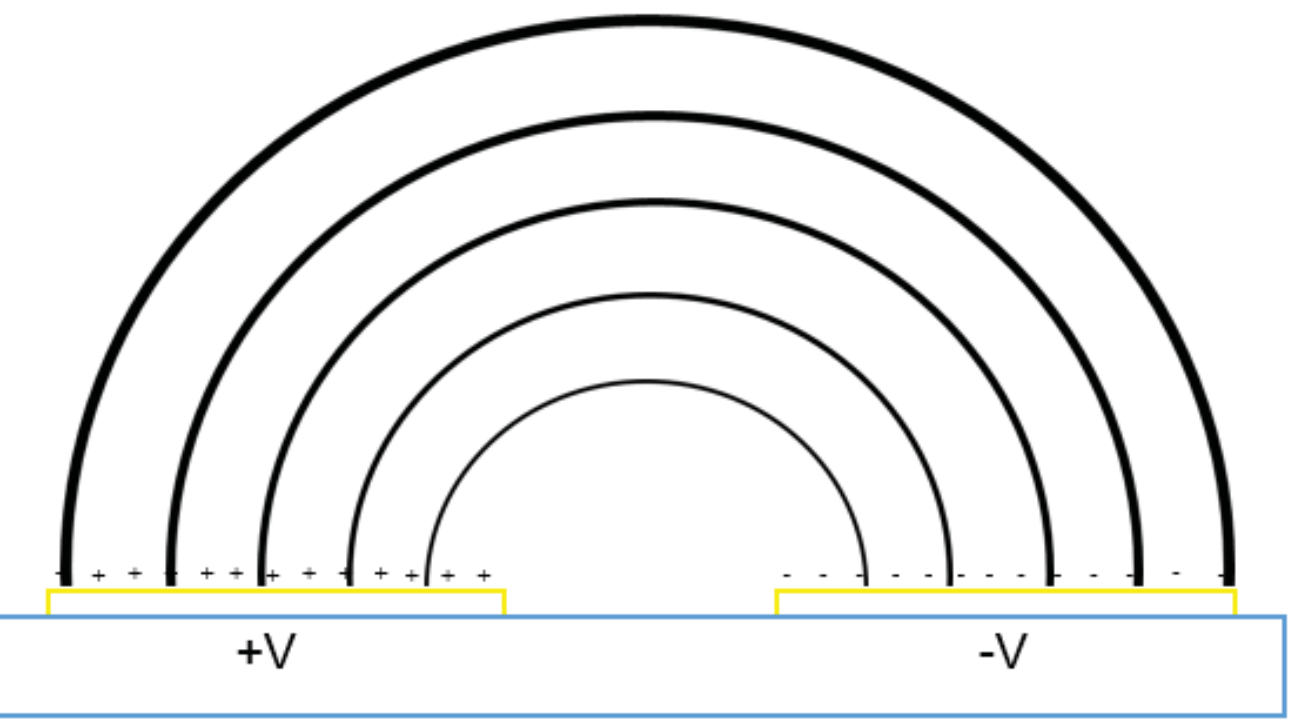

Figure 3: Schematic diagram showing fundamental principle of ACEO. The blue rectangular represents the glass slide, the yellow rectangular represent gold electrode. Black lines in circles are electric field lines

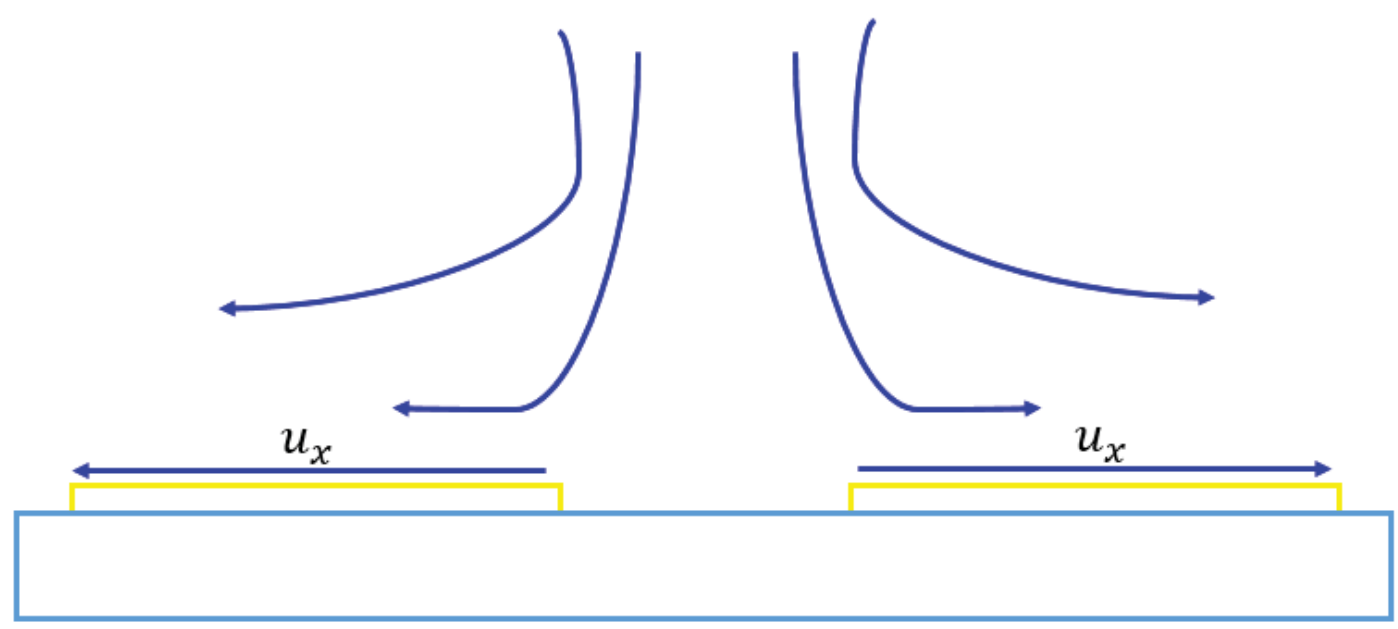

Figure 4: Schematic diagram showing flow pattern in ACEO system. 
The ACEO induced flow is occurring in the pattern of recirculation [36-39]. In order to generate net directional fluid flow to apply this concept into micropump applications, multiple methods have been reported to break the symmetric property of the flow. Geometrical non-symmetric electrode pair was used to generate non-uniform electric field and non-uniform electrode polarization [40] [41]. DC-biased AC signal was also used to change the electrode polarization symmetric condition thus induce net flow [42] [43]. Travelling-wave AC electric potential with different initial phases

are also proved to be efficient in inducing net flow in ACEO system [44]. Chemical treatment on electrode surface was also proved to be able to induce net flow by changing the electrode charging condition [45].

\subsubsection{Alternating Current Electrothermal Flow (ACET)}

Joule heating is a phenomenon in which heat is generated when electric current flows through electrically conductive fluid. Such Joule heating effect combining with physics from electrical, thermal and fluidic mechanics rises the phenomena of AC Electrothermal (ACET) flow [22] [46].

Electric current density $\vec{J}$ is established once electric potential is applied across the electrolyte solution with the magnitude of:

$$
\vec{J}=\sigma E=\left(\mu_{+}+\mu_{-}\right) c \vec{E}
$$

where $\sigma$ is electrolyte solution conductivity, c is electrolyte solution concentration, $\mu_{+}$and $\mu_{-}$are the limiting ion conductivities for anions and cations at $298 \mathrm{~K}$. 
The passage of such current through the electrolyte solution thus is inducing the Joule heating effect generating heat. The temperature change $\Delta T$ raised from such Joule heating effect thus can be estimated as:

$$
\Delta T \approx \frac{\sigma V_{r m s}^{2}}{k}
$$

where, $V_{r m s}$ is the root mean square of applied potential and $k$ is the thermal conductivity. In uniform electric field, $\Delta T$ is spatially uniform and solution properties such as permittivity and conductivity are also changed uniformly thus no net force is induced on fluid. In nonuniform electric field shown in Fig. 5 [46], however, spatial variation of fluid permittivity and conductivity is raised by non-uniform temperature change. Such spatial variation then induced a net force $f_{e}$ on the fluid with the general equation of [47] [48]:

$$
f_{e}=\rho_{q} E-\frac{1}{2} E^{2} \nabla \varepsilon+\frac{1}{2} \nabla\left(\rho_{m} \frac{\partial \varepsilon}{\partial \rho_{m}} E^{2}\right)
$$

where $\rho_{q}$ is volume charge density. 


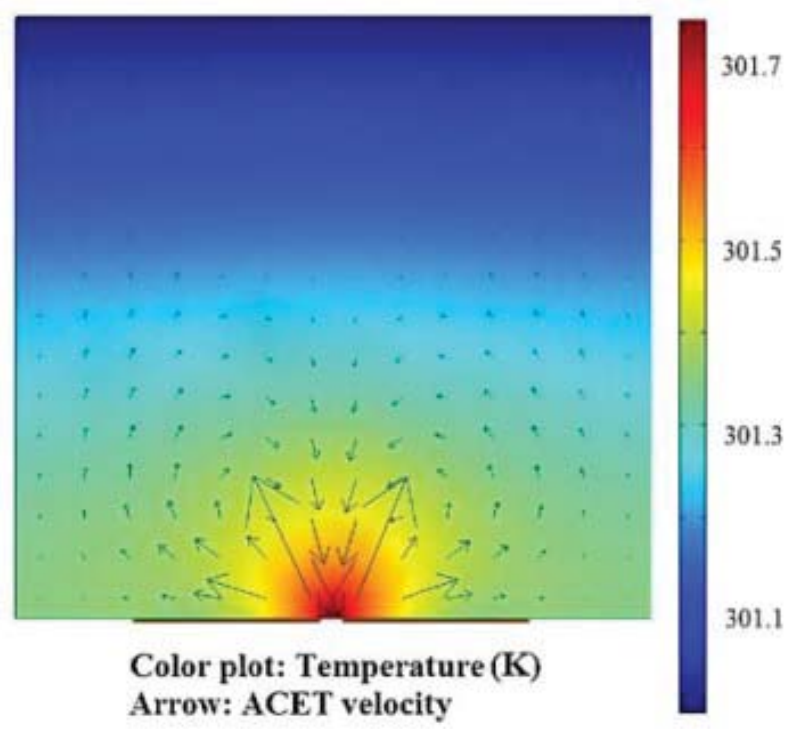

Figure 5: Simulated ACET flows: color plot shows temperature and arrows show flow velocity [46] (Figure reproduced with permission)

As a result, in major AC electrokinetics including DEP, ACEO and ACET, both electric field spatial non-uniformity and medium electrolyte solution property are very important. The spatial non-uniformity of electric field gives a non-zero DEP force on dielectric particle and net motion in ACEO as well as spatially varied temperature change inducing ACET. The medium electrolyte solution concentration, electric permittivity and conductivity are also determining the direction and magnitude of DEP force and velocity and frequency range for both ACEO and ACET solution.

The electrolyte properties are normally assumed to be constant under $\mathrm{AC}$ electric field due to the oscillating nature of the signal. However, few works have examined the validity of this assumption, especially under non-uniform electric field in microfluidic systems. In this dissertation, such assumption is proved to be invalid by dividing and detecting each term in 
electrolyte transport equation and the frequency and potential dependencies are also examined. Chapter 2 reviews published works related to the electrolyte transport term-by-term including Faradaic reaction, electromigration and convection. Chapter 3 and 4 have been published on Biomicrofluidics and Chapter 5 is also going to be published. More specifically, chapter 3 demonstrates the experimental work on the observation of solution biocompatibility change issue in DEP field, which initiated the idea for this dissertation. Chapter 4 demonstrates the experimental work on detecting the Faradaic reaction effect on medium solution under non-uniform $\mathrm{AC}$ electric field. Chapter 5 demonstrates the experimental work on detecting the electromigration effect on medium solution under non-uniform AC electric field. Chapter 6 includes the conclusions and planned future works. 


\section{CHAPTER 2 BACKGROUND AND REVIEW OF IONIC SOLUTION ELECTROCHEMISTRIES UNDER AC POTENTIAL}

\subsection{Introduction}

It has been demonstrated in the introduction section that almost all electrokinetic responses of particles or fluids such as EP, DEP, ACEO and ACET are highly dependent on media solution condition in microfluidics systems. However, such importance of solution properties are rarely addressed in published papers. Few published works demonstrated solution property changes induced by Faradaic reaction (electron transfer between electrode and electrolytes) under DC and AC potentials in microfluidics. Even fewer related works have been published on ion transport induced by Ohmic current affected by electric field in bulk electrolyte, especially under high frequency AC electric fields. Instead, such Faradaic reaction or ion transport are normally arbitrarily assumed to be negligible under high frequency AC signals and were not considered in most of works.

The following background and literature review focuses on electrolyte solution electrochemistry behaviors including both non-Faradaic processes and Faradaic. First, general non-Faradaic process including electromigration, diffusion and convection are discussed under DC signals. Then, Faradaic properties under DC signals are discussed shortly, with the emphasis on the relationship between Faradaic and non-Faradaic process. After that, major impacts from AC signals on Faradaic reactions are described, with examples from published literatures. At last, knowledge on non-Faradaic process 
expanding to AC signals are also described. Finally, the reported impacts from both Faradaic and non-Faradaic processes on microfluidics are demonstrated, with emphasis mainly on AC cases.

\subsection{Non-Faradaic Processes}

The non-Faradaic process describes the charge transfers that does not induce Faradaic reaction, i.e., the transfer of electron between electrode and electrolyte. Adsorption and desorption are two examples for such non-Faradaic process at electrode-electrolyte surface and such processes can also change with externally applied potential and solution conditions. Although the non-Faradaic process itself does not carry any Faradaic reaction, it still determines the charge transfer process, which would further affect Faradaic reactions. Also, the observation of impact from non-Faradaic process under $\mathrm{AC}$ electric field is one of the major goals of this dissertation. As a result, non-Faradaic process under DC potential is reviewed as the first part in this section.

In non-Faradaic processes, the electrodes are usually referred as ideal polarized electrode (IPE)', on which no charge transfer can process between the electrode-electrolyte interface. Once electric potential is applied on IPEs,

there are charges established on electrode surface, with amount of $\mathrm{q}^{\mathrm{M}}$ and the electrostatic forces attract the counter-ions towards the electrode with amount of $\mathrm{q}^{\mathrm{S}}$. However, due to the nature of IPE, no charge transfer would occur between the attracted ion and electrode, which is resulting in a capacitor behavior of electrode-electrolyte interface. When ions travel to the electrode, they tend to accumulate on electrode surface and form a layer with excess ions 
with the total charge of $\mathrm{q}^{\mathrm{S}}$. Such layer of charged ions and oriented dipole molecules is then called electrical double layer, which is normally represented by a capacitance, $\mathrm{C}_{\mathrm{d}}$, or $\mathrm{C}_{\mathrm{d} \text { l. }}$. Since such electrical double layer capacitance is established by the induced electrical double layer, the capacitances is often a function of applied electrical potentials. Figure 6 shows the generally agreed model for the electrical double layer $[49,50]$.

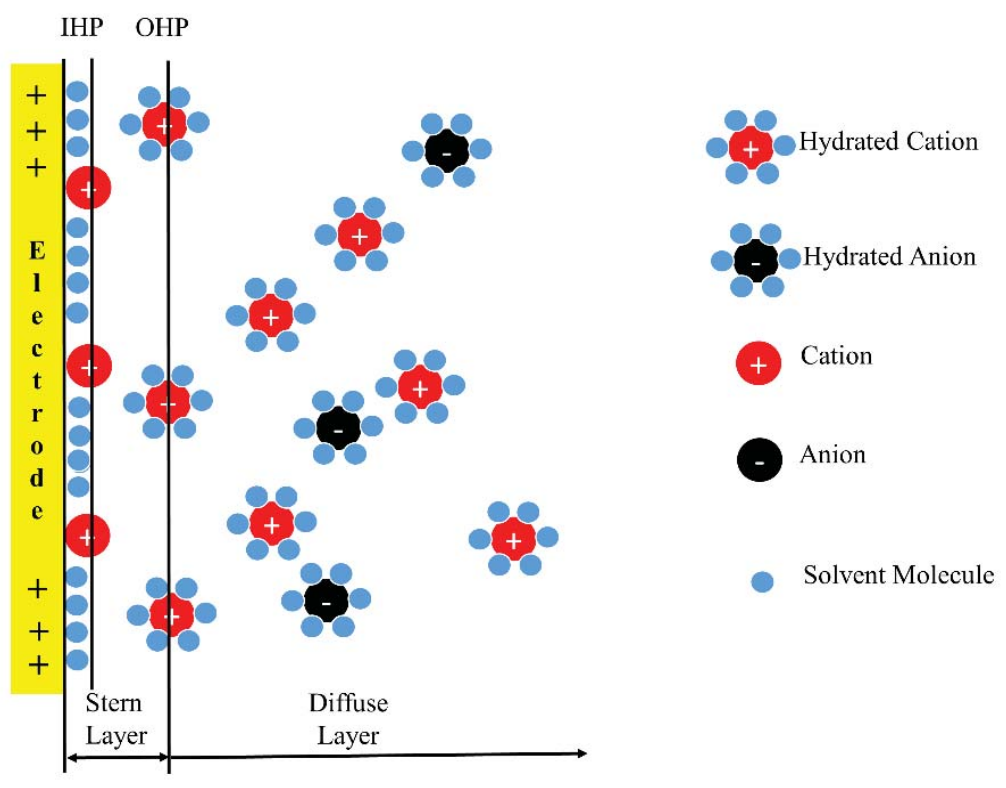

Figure 6: Diagram of electrical double layer configuration on a positively charged electrode. IHP and OHP stands for inner and outer Helmholtz plane, respectively

The first layer on the electrode is called inner Helmholtz plane (IHP), which includes dielectric solvent molecules and specifically adsorbed cation or anion. Specific adsorption is induced by short-range interaction between electrodes and molecules. The specifically adsorbed molecules are strongly bonded to electrode surface and such bonding does not depend on the electrode charging condition, which means both cations and anions can be 
specifically adsorbed onto either positively and negatively charged electrodes. Such adsorption majorly depends on electrode material and cation and anion species.

Outside the inner Helmholtz plane is the outside Helmholtz plane (OHP). The position of OHP is determined by the center of solvated ions that are nearest attracted onto the electrodes. Different from specifically adsorbed IHP layer, solvated ions in OHP layer are said to be nonspecifically adsorbed, which means they are independent of the chemical properties of electrode material or ion species. Instead, the long distance electrostatic forces are the causing reason for them to be attracted onto electrodes. Thus, cations and anions can be only attracted when electrodes are negatively or positively charged, respectively.

Further away from the electrode surface, the effects from ions thermal agitation starts to be prominent. The nonspecifically adsorbed ions are then in quasi-equilibrim state under a force balance between the long-distance electrostatic force and the thermal agitation force, staying in the so-called diffuse layer, which start from OHP and extends to the bulk electrolyte. 


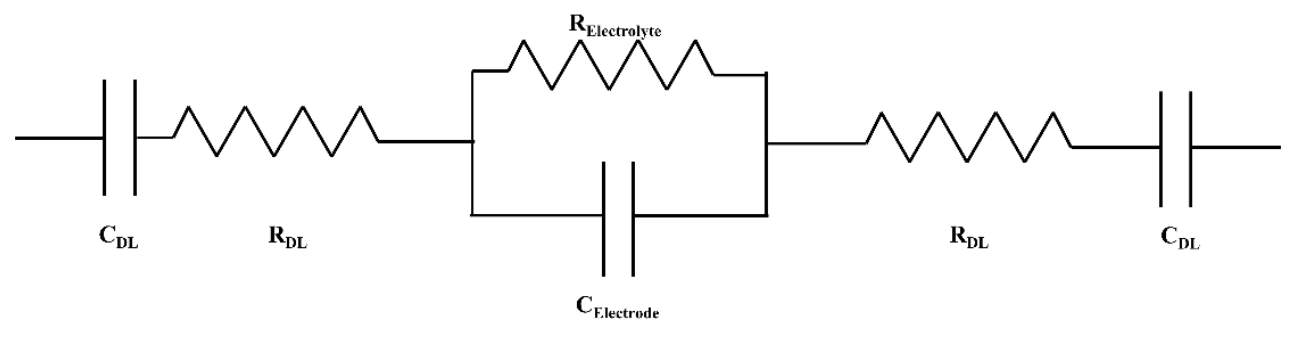

Figure 7: Simplified circuit for electrolyte between two electrolyte. Bulk electrolyte is considered as RC parallel circuit and electrical double layer is considered as RC series circuit on each side of electrodes

Outside of the diffuse layer (opposite direction of electrode) is bulk electrolyte where equal number of cations and anions exists and the general bulk solution is neutral. In bulk solution, ion flux was induced by convective flow and difference of electrochemical potential including potential difference induced by electric potential and by chemical concentration gradient. Bard and Faulkner have given implicit explanation of the mechanisms of ion transport in electrolyte [51].

Chemical species j's flux is induced by the gradient of electrochemical potential $\left(\overline{\mu_{\jmath}}\right)$

$$
J_{j} \rightarrow \nabla \overline{\mu_{J}}
$$

in which,

$$
\overline{\mu_{J}}=\mu_{j}^{0}+R T \ln a_{j}+z_{i j} F \varnothing
$$

and the proportionality coefficient of species flux to the gradient of electrochemical potential is $-C_{j} D_{j} / R T$, where $\mathrm{R}$ is gas constant, $\mathrm{T}$ is 
temperature, a is species activity, $\mathrm{z}$ is species valance, $\mathrm{F}$ is Faraday's constant and $\varnothing$ is electric potential. Taking convectional term into consideration gives:

$$
J_{j}=\frac{-C_{j} D_{j}}{R T} \nabla \overline{\mu_{j}}+C_{j} v
$$

where, $\mathrm{C}$ is species concentration, $\mathrm{D}$ is species diffusion coefficient and $\mathrm{v}$ is convective velocity.

Then, the Nernst-Planck equation can be obtained as:

$$
J_{j}(x)=-D_{j} \frac{\partial C_{j}(x)}{\partial x}-\frac{z_{i} F}{R T} D_{j} C_{j} \frac{\partial \varphi(x)}{\partial x}+C_{j} v(x)
$$

Combing the Nernst-Planck equation with the mass conservation gives the time evolution of ion concentration:

$$
\frac{\partial C_{j}}{\partial \boldsymbol{t}}=D_{j} \nabla^{2} C_{j}+\frac{z_{i} F}{R T} D_{j} C_{j} \nabla^{2} \emptyset-C_{j} \boldsymbol{v}+R_{j}
$$

where, $\mathrm{t}$ is time and $R_{i}$ is the reaction rate for species $\mathrm{i}$.

Such equation is often calculated in combination with Possion equation to take into account the effects from the ion migration to the electrical potential in return:

$$
\begin{aligned}
& \nabla \cdot\left(\varepsilon \varepsilon_{0} \nabla \emptyset\right)=-\rho_{e} \\
& \rho_{e}=\left(c^{+}-c^{-}\right) z e
\end{aligned}
$$

where, $\rho_{e}$ is charge density, $c^{+}$is cation concentration and $c^{-}$is anion concentration. Bulk electrolyte solution is normally regarded as electroneutral so $\rho_{e}=0$. Combing Eqn. 2.6 with electroneutral assumption with Eqn. 2.5, under the assumption of 1:1 symmetric electrolyte, the ion/electrolyte transport can be then derived into the equation of convective diffusion, with further derivation can be found[52]:

$$
\frac{\partial \mathrm{C}}{\partial \mathrm{t}}=D_{e f f} \nabla^{2} C
$$

where, 


$$
D_{e f f}=\frac{1}{\left(\frac{z_{+}}{z_{+}+z_{-}}\right) \frac{1}{D_{-}}+\left(\frac{z_{-}}{z_{+}+z_{-}}\right) \frac{1}{D_{+}}}
$$

Eqn. 2.8 implies that at steady state, in the absent of electrokinetic current, cations and anions will respond to the electric field as one species under the impacts from electroneutrality. However, this phenomena only occur at certain finite time after application of the electric field. At the moment electric field is applied, cation and anion tend to separate in response to the electric field. The separated ions then breakdown the local electroneutrality even in bulk solution. However, when ions are further separated, a potential drop between the separated ions is established in companion with the internal electric field. Such induced internal electric field then counteracts the effect of externally applied electric field and keeps the net field in solution to be zero to maintain the macroscopic electroneutrality.

As a result, in DC condition, exchange of charge carrier from electron to ions at the electrode-electrolyte interface is necessary to maintain closed loop of the electrochemical cell. However, since the establishment of internal electric field requires finite time, ions oscillate in response to the $\mathrm{AC}$ fields thus the existence of current becomes possible even in pure Faradaic processes.

\subsection{Faradaic Processes}

The Faradaic process describes the transport of charge/electron between an electrically conductive subject, such as electrode, and ionic conductive subject, such as electrolyte solution. Such Faradaic process causes oxidation 
or reduction to occur on different electrode surfaces and is governed by Faradaic law ('the amount of chemical reaction caused by the charge flux is proportional to the amount of electricity passed'). There are two types of electrochemical cells could go through such Faradaic process. Galvanic cell is the one that Faradaic process occurs spontaneously once the electrochemical cell form a closed circuit by external connected conductor. Electrolytic cell is the one that Faradaic process occurs passively by application of external potential. Since the major concern in this dissertation is on electrokinetics, following reviews will be focused on electrolytic cells. Also, since the major concern in this dissertation is under $\mathrm{AC}$ condition, where the transport of ions is greater concern rather than the electrochemical reaction kinetics, more emphasis would be put on the ion and electron transfer aspect of the reaction problem rather than the thermodynamics and kinetics.

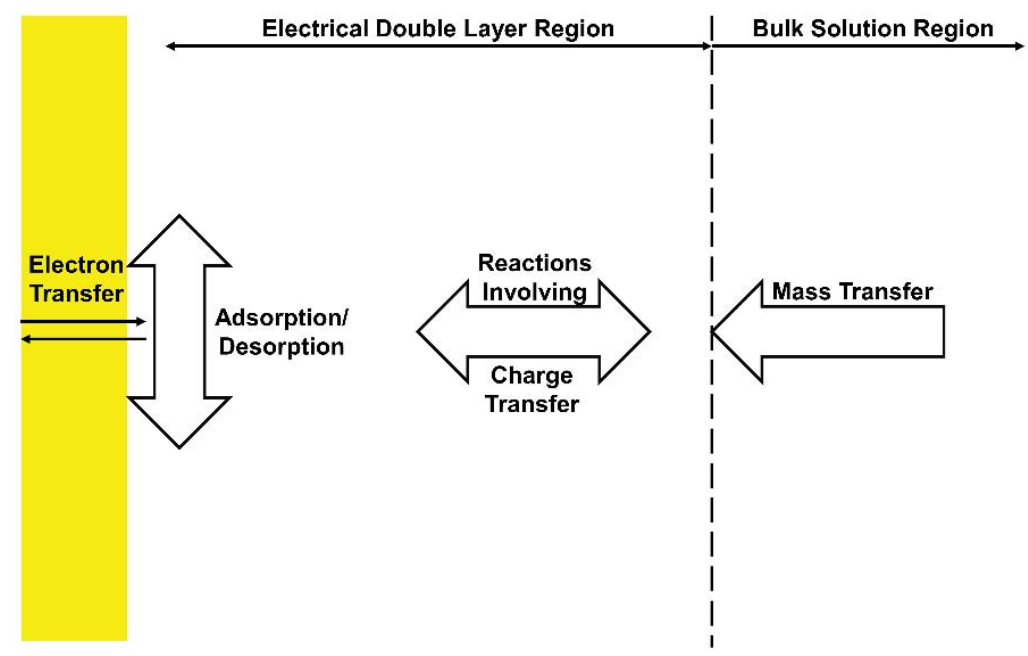

Figure 8: Process of electrochemical reaction: from bulk solution to electrical double layer to electrode surface

Fig. 8 demonstrates the simple form of whole process for electrochemical reaction. When no external potential is applied on the electrode, the system 
majorly stays in homogeneous phase with only minor exchange current occurring on electrode surface due to inevitable spontaneous reactions. Once the potential is applied, for example, a negative potential, cations in bulk electrolyte then transfer towards the charged electrode into the electrical double layer. Then, electron transfer occurs between electrode and electrolyte inside the electrical double layer, under the condition that electrode is polarized, which by definition means there is a difference of potential from the equilibrium value upon passage of Faradaic current on the electrode (the extent of polarization is measured by the overpotential). Such transfer of electron then induces chemical reaction of the cations (such as reduction reaction induced by losing electron). After that, the reduced molecule can process surface reactions such as adsorption, desorption or crystallization.

\subsection{Faraday Processes under AC Electric Potential: Potential Drop and Experimental Work}

Grahame's review in 1955 [53] discussed specifically about the relationship between electrical double layer and Faradaic process. He claimed that 'the reduction of an ion takes place, not from the interior of the solution, but from the electrical double layer'. As a result, for Faradaic processes, the effective potential facilitating electron transfer between electrode and electrolyte is the potential drob between the electrode-electrolyte interface and outer Helmholtz plane shown in 8. Also, the real concentration of ions participating the electrochemical reaction should be the concentration at outer Helmholtz plane instead of the bulk electrolyte concentration. More detailed mathematical expressions description about application of proper electrical potentials and ion concentrations can be found in some references papers of 
Grahame's review [54-56].

Under the impact from DC potential, the potential drop across the double layer and ion concentration inside the double layer can be obtained from the knowledge of zeta potential, which is describing the electric potential on the double layer - solution interface $[57,58]$.

Under the impact from AC potential, however, the potential across the electrical double layer providing the overpotential for Faradaic processes is a function of applied signal frequency. Because of the finite number of ion mobility and the importance of diffusion effect, the establishment of electrical double layer takes finite amount of time, which is called the characteristic time or electrical double layer charging time $t_{c}$ which is given by the product of typical resistance of bulk electrolyte [59] $L / \sigma S$ and the typical capacitance of EDL $\varepsilon S / \lambda_{D}$ :

$$
t_{c}=\left(\frac{\varepsilon}{\sigma}\right)\left(\frac{L}{\lambda_{D}}\right) \quad \text { and } f_{c}=\frac{1}{t_{c}}
$$

where, $\varepsilon$ and $\sigma$ are the permittivity and conductivity for the fluid. $L$ and $S$ are the characteristic length and area for the system. $\lambda_{D}$ is the Debye length measuring the distance between charged surface and the outer boundary of diffusion layer which for the monovalent electrolyte can be expressed as:

$$
\lambda_{D}=\sqrt{\frac{\varepsilon R T}{2 F^{2} C_{0}}}
$$

where, $R$ is gas constant, $T$ is temperature, $F$ is Faradaic constant, $C_{0}$ is bulk molar concentration and the $f_{c}$ is electrode characteristic frequency, which equals to the inverse of charging time, $t_{c}$. 
Under the alternating applied potential, there is finite time below which it is not sufficient for the double layer to establish in each half cycle. At very low frequencies, $f \ll f_{c}$, there is enough time at each half period of the applied signal for the electrical double layer to be well established and the EDL under such low frequency AC field behaves similar to DC condition, only in an alternating way and the potential drop is still majorly across the electrical double layer, resulting in the ample potential provided for the Faradaic processes. At very high frequencies, $f \gg f_{c}$, there is no time for the electrical double layer to be established and the potential drop thus falls across the bulk electrolyte solution. Under this condition, according to Grahame's review, there is no potential applied across the electron-electrolyte transfer region, which is the electrical double layer thus the Faradaic reaction rate should be negligible. At moderate frequencies, $f \approx f_{c}$, the electrical double layer is partially established at each half applied cycle and the potential drop is then shared by the bulk electrolyte solution and electrical double layer. Under this condition, the potential drop for the Faradaic process is decreased resulting in a decreased Faradaic reaction rate. A general RC circuit shown in Fig. 6 can facilitate the understanding for the potential drop between double layer and bulk electrolyte. However, such figure only expresses the ideal behavior of the double layer, whereas it has been proved that the double layer capacitance is also a function of applied frequency and strength of electric field [60].

Ramos et al. derived a simplified equation for potential across the double layer under the assumption of the applied frequency is much smaller than the ion relaxation frequency [36], $\omega \ll \sigma / \varepsilon$ : 


$$
\Delta \emptyset_{D L}=\frac{V_{0}}{2+i \omega \pi\left(\frac{\varepsilon}{\sigma}\right) \kappa x}
$$

where, $\kappa$ is the inverse of thickness of electrical double layer and $\mathrm{x}$ is the distance from electrode in $\mu \mathrm{m}$. The potential drop on bulk electrolyte solution is then:

$$
\Delta \emptyset_{B u l k}=1-\Delta \emptyset_{D L}
$$

Such potential drop across the bulk electrolyte is a complex number, thus the real part of the generated electric fields correlates to dielectrophoresis phenomena and complex part of the electric fields can produce travelling wave effects. They have also plotted figures in [23] page 105 Figure 6.12 (a) and (b) which demonstrate relationship between potential, frequency and position in bulk electrolyte for the real and imaginary part of potential.

Most of the published experimental works on AC electrolysis were in early 1900s. The origin of AC electrolysis can be traced back to 1719 . Manuevrier and Chappius noticed an evolution of gas when dipped a very fine platinum wire into sulfuric acid solution under alternating current, where the amount of generated gas decreased with increase of applied frequency.

Similar trend was observed by Leblanc in 1905 when he was applying AC current from 16.7 to $634 \mathrm{~Hz}$ on copper electrode with cyanide solution. He observed significant copper electrolysis under $16.7 \mathrm{~Hz}$ while only about one third of the effect can be achieved under $634 \mathrm{~Hz}$.

Ayrton and Perry applied AC current at $167 \mathrm{~Hz}$ on platinum electrodes in water system. Based on their observation, hydrogen gas deposition on 
platinum electrode decrease the extent of electrolysis by AC current while oxygen gas deposition increase such extent. However, such observation of frequency dependence of reaction rate was attributed to the lack of time for copper to be converted to other chemical compound in bulk solution, which is different from theoretical explanation of modern theory.

A theoretical explanation match fits better into modern electrochemistry was firstly proposed by Brochet and Petit. They believed the formation of new compound is at the time point when the ion reaches the electrode and had enough time to discharge before reverse reaction at the same electrode.

AC electrolysis is also studied on a corrosion problem on the single phase railway motor design [61]. Hayden performed tests on AC electrolysis on multiple electrolytes based on combination of Ammonium, Nitrate, Sodium, Potassium, Chloride and Sulphate ions. Lead and iron plates were used as electrodes. Instead of constant potential, he has applied constant current of 0.3 and 0.09 amperes (A) for different time duration of $\sim 70,140$ and 150 hours. For results part, he measured parameters including electrode dissolved per hour, percentage of current effected on electrolysis, ratio of total corrosion to chemical corrosion and ratio of current to frequency.

Four remarkable conclusions were drawn from this work. $1^{\text {st }}$, Hayden claimed that the $\mathrm{AC}$ electrolysis cannot be quantified as DC case since the $\mathrm{AC}$ electrolysis has the character of secondary effects, which means the reaction rate at each half cycle is different leaving a small difference within $1.5 \%$. 
$2^{\text {nd }}$, according to the experimental conditions, $\mathrm{AC}$ electrolysis behaves very differently from DC electrolysis. He concluded that nitrate based compounds tend to increase electrolytic corrosion as fertilizers (helpers) whereas carbonates decreased the corrosion as a protectorate.

$3^{\text {rd }}, \mathrm{AC}$ electrolysis also depends significantly on the temperature. The increase of temperature gives 1 higher reaction rate which is represented by higher corrosion rate in his work.

$4^{\text {th }}, \mathrm{AC}$ electrolysis reaction rate decreases with frequency, represented by the decrease of corrosion rate in his work. This observation also experimentally matches the theoretical works which claims that high frequency AC signal decreases the potential drop on electrical double layer thus decreases the reaction rate.

Although Hayden's work is focused on detection of corrosion properties, the fundamental concept of AC electrolysis was suggested and large amount of experimental evidences can be matched by modern theoretical works generated in recent years.

Marsh et al. in 1919 worked on an electrochemical cell originally designed for bacteria research, the equipment used in this work is very similar to the ones used in modern microfluidics. Due to the observation of bubble formation on electrode and failures generated on the bacteria experiments, Marsh focused on the detection of AC electrolysis phenomena in electrochemical system for biological research. He tested the AC electrolysis 
over electrodes including platinum, gold and Nickel and electrolytes including diluted Sulphuric acid and Barium Hydrate under 1 A current and frequency from 25 to $80 \mathrm{~Hz}$. They are still measuring the generated gas volume in order to measure the reaction intensity. The results demonstrate that the electrolysis follows similar pattern of first order reaction under all frequencies, where the amount of reaction product increases with time while reaction rate decreases with time. Similar frequency dependency can be also observed in this work where the amount of bubbles generated decreases with increase of frequency.

In 1927, Allmand and Cocks [62] applied the similar method of measuring gas volume to determine current efficiency and Faradaic reaction rate. More importantly, the electrolyte they have inspected was Potassium Chloride, which is one of the most commonly used electrolyte in modern microfluidics systems. They have applied constant current on both graphite and platinum electrodes at frequencies range from 6 to $18 \mathrm{~Hz}$. They have also inspected reaction property for Potassium Chloride with initial concentration of 1 and $3 \mathrm{M}$.

For graphite electrodes, only very weak reaction was observed at $6 \mathrm{~Hz}$ at $1 \mathrm{~A} / \mathrm{cm}^{2}$ and no higher current was examined in order to protect electrode. However, significant reaction was observed at $6 \mathrm{~Hz}$ on platinum electrode even at $0.3 \mathrm{~A} / \mathrm{cm}^{2}$ and Hydrogen, Hypochlorite and Oxygen was reported as reaction products.

Fig. 10 shows another behavior similar to DC electrolysis was observed during the detection of current dependency. It can be observed that at low or 
moderate frequency of 6 and $22 \mathrm{~Hz}$, an abrupt change of current efficiency existed at 0.3-0.4 and 1.0 Hz, respectively. Such observation then matched the polarization behavior under DC condition and the overpotential theory, where current stayed almost zero below the overpotential and increased significantly with the increase of potential above the overpotential.

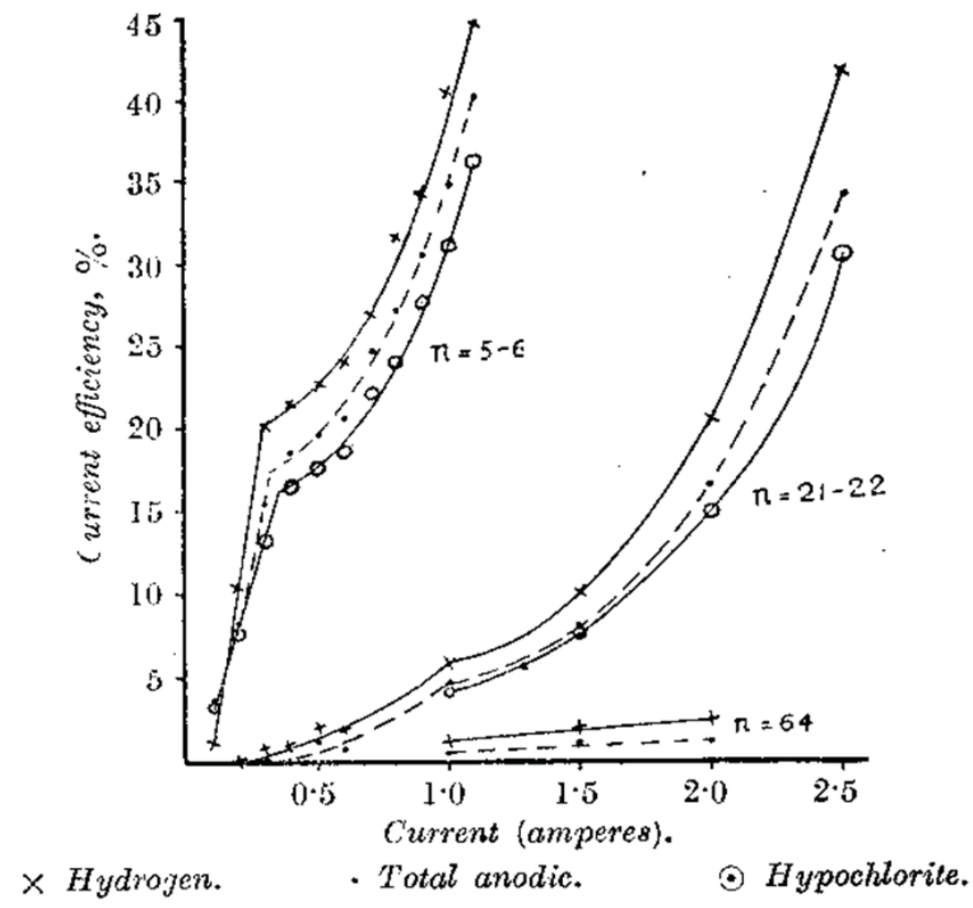

Figure 9: Output current efficiency dependence on input current at frequencies of 5-6, 21-22 and $64 \mathrm{~Hz}$ [62]. (Reproduced with permission)

Although there are some similarities between AC and DC electrolysis discussed above such as similar overpotential pattern and similar first order reaction behavior, a major discrepancy on the reaction magnitude exists between AC and DC electrolysis. It was observed that even under the similar applied potential (i.e. $2 \mathrm{~V}$ rms in AC condition and $2 \mathrm{~V}$ DC condition), the effect of AC electrolysis is weaker than DC case. 
A remarkable theoretical explanation was proposed by Shipley [63] in 1929 attributed such observation to the 'back electromotive force' using an example of AC water electrolysis. Sinusoidal external current was applied $\mathrm{f}$ as a full cycle, with a total of $4 \mathrm{q}$ coulombs charge passing through the whole cycle. First of all, ions were attracted to the electrode due to electrostatic force and formed electrical double layer. Also, a reaction current would be induced if the peak potential at point $\mathrm{A}$ is greater than the Faradaic process overpotential, resulting in the conversion of hydroxyl ions to water and oxygen. The produced oxygen then could interact with metal electrode by either processing reaction or, more likely, be adsorbed or absorbed on the electrode surface or in the volume, with the quantity of qox shown described by the coulombs of electrons participated in the reaction producing the molecules. The remaining oxygen then would be eliminated from the electrode, thus, can be represented by q- qox. Signal with opposite sign was applied from O-C-D. However, before the production of hydrogen gas, the stored oxygen at point $\mathrm{O}$ was producing a 'back electromotive force'. Due to the stored oxygen, hydrogen with amount of $\mathrm{q}_{\mathrm{ox}}$ would firstly react with the stored oxygen. Then the electrode could be restored to neutral condition and process the reaction and adsorption of hydrogen, at the magnitude of $\mathrm{q}_{\mathrm{h}}$. As a result, in AC electric field, the real amount of oxygen or hydrogen gas is always reduced by the remained gas from previous half cycle, and a discrepancy is thus generated between the AC and DC case.

Compiling the above information, it can be concluded that $\mathrm{AC}$ electrolysis generally follows the same principle and same pattern of the 
traditional DC electrolysis. Ideally, in each half cycle, ions are attracted to the electrode. After that, electrode-ion electron transfer is processed in similar pattern to DC condition indicated by the suddenly increased current efficiency from Fig. 10. Also, the reaction kinetic under AC electric field still follows the similar pattern to the traditional first order reaction with the reaction rate decrease with increase of time. The only major difference is the additional frequency dependence, which decreases the magnitude of Faradaic process by introducing the 'back electromotive force' induced by reaction product adsorption/absorption on electrode. Also, the Faradaic reaction rate is inversely correlated with frequency, with reaction rate decrease with increase of frequency.

\subsection{Non-Faraday Processes under AC Electric Potential: Double Layer and Bulk Electrolyte}

Non-Faraday processes in both electrical double layer and bulk electrolyte were included in almost all electrochemical systems such as fuel cells [64] [65], lithium battery [66] [67] etc. and numerous works have been published on such topic, especially under DC electric field [68-76].

\subsubsection{Non-Faraday Processes under AC Electric Potential within Double Layer}

Non-Faraday processes on electrode surface under AC electric field have also been well studied in traditional electrochemical cells. One of the most common application of AC potential is AC voltammetry, where a DC signal is superimposed on a small AC signal. However, such superimposed signal deviates from the original 'AC' concept and in fact is an 'oscillated DC' signal 
in strict definition where the applied potential changes with time but always greater or less than zero volt or 'biased AC' signal.

The major application for the AC non-Faradaic, although still in combination of Faradaic process, is the electrochemical impedance spectroscopy (EIS) system, which is designed for high precision measurement of charge transfer parameters and for studying the structure of electrical double layer. In EIS system, an AC signal with very small amplitude is applied to an electrochemical cell, which can be represented by equivalent circuit such as Fig. 7. The output current thus change almost linearly with amplitude of potential as well as frequency due to the small amplitude of applied signal. Under that linear area (i.e., small applied signal, infinitely thin compacted layer etc.), a frequency sweep is applied to the electrochemical cell and real and imaginary parts of the total cell impedance can be obtained. Such obtained impedance then can be used to characterize system kinetic and dynamic properties.

When the applied potential is slightly higher than the system overpotential, output current signal is induced by both electrical double layer capacitor representing non-Faradaic process and charge transfer representing Faradaic process. However, when the applied potential is lower than system overpotential, the thermodynamic barrier of electrochemical reaction is not able to be passed so no Faradaic process exist in the system. As a result, the output current is induced by only non-Faradaic process such as electrical double layer and ohmic conduction. 
EIS technique is also widely used in electrokinetic microfluidic systems. Impedance spectroscopy for single cell measurement provides cell physiological property $[77,78]$. Cell viability and concentration in certain solution can be also determined by data from impedance spectroscopy [79]. Hong et al. [80] also applied EIS data in designing of interdigitated electrodes, such as electrode length, width and gap by fitting impedance data similar to Fig. 11 to the equivalent circuit in Fig. 7.

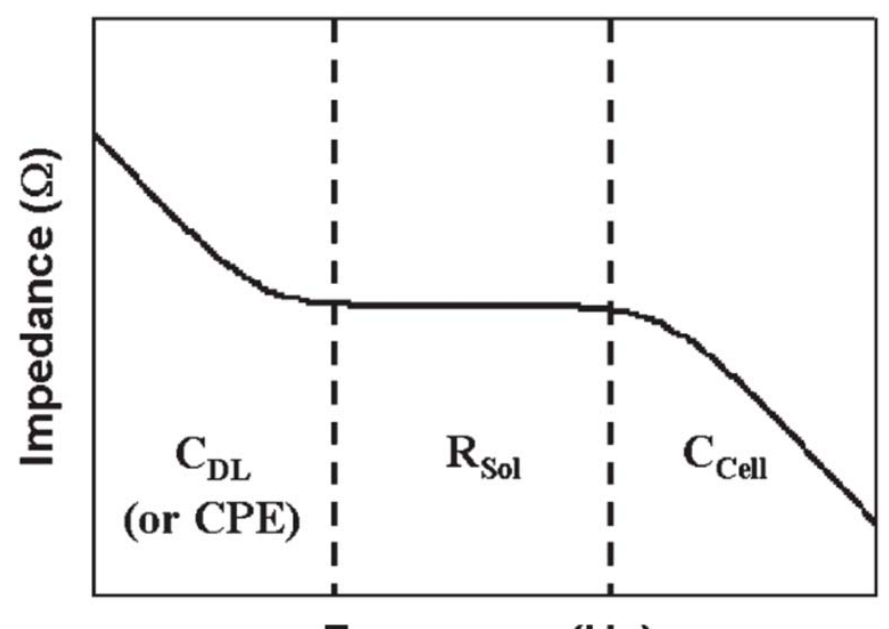

Frequency $(\mathrm{Hz})$

Figure 10: Schematic diagram of impedance spectroscopy for interdigitated electrodes [80] (Reproduced with permission)

The two dashed lines in Fig. 11 show the lower and higher cut-off frequency, respectively. Electrical double layer capacitor dominates the impedance response below the lower cut-off frequency while cell capacitor by the electrolyte dominates above the higher cut-off frequency. Also, the equivalent circuit shows resistance behavior between the higher and lower cut-off frequencies. According to the reading of the cut-off frequency and solution impedance, the cell and double layer capacitance can be obtained and 
facilitate the design of electrode geometry and electrolyte condition optimization[81].

Experimental work on directly detection electrical double layer properties has also been accomplished by Singh et al. [82]. They reported data obtained from high sensitive differential interferometry operated by laser beam of ion concentration gradient in electrical double layer with respect to signal magnitude and frequency. As shown in Fig. 11, the interferometry is measuring the amplitude of path length modulation. As the ion accumulation happens at the electrode-electrolyte interface of working, floating and cage electrode, the refractive index will change and generate a modulation in the beam path of the two laser beams and the difference modulation can be obtained by the difference from working and reference electrode (caging electrode). 


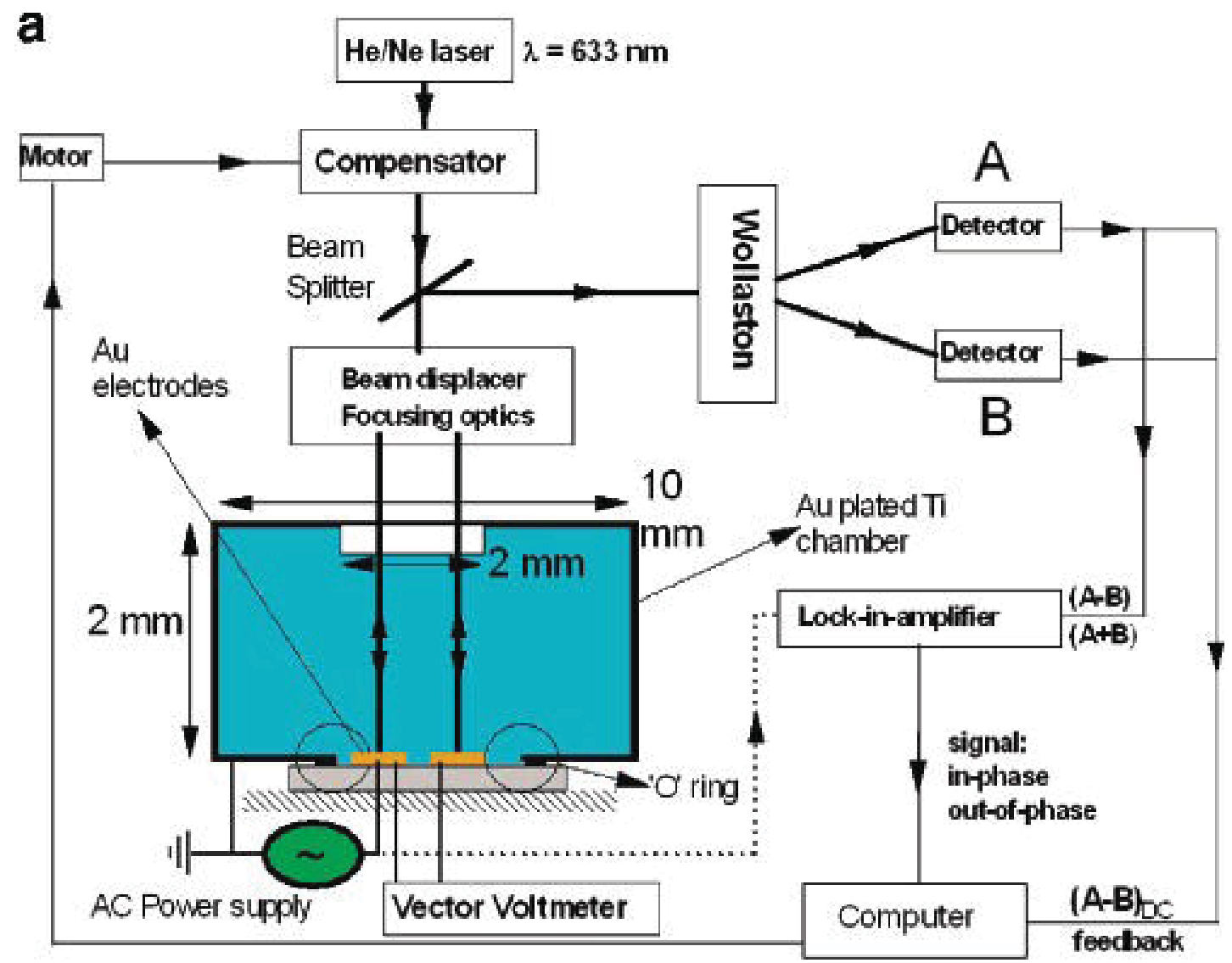

b

\section{Equivalent Circuit}

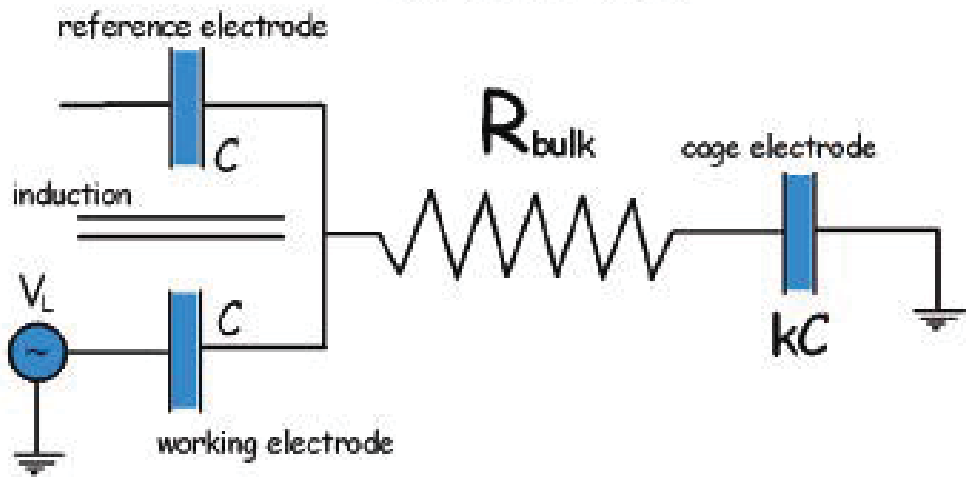

Figure 11: Diagram of differential interferometric system designed for measurement of electrical double layer [82] (Reproduced with permission)

Result from such system verified that most of the ion accumulation in the electric double layer was linear up to high electrode potential and salt 
concentration. Ion concentration gradient in electrical double layer increased with increase of both initial bulk electrolyte concentration and externally applied electrical potential.

Fig. 12 is showing the dependency of absolute modulation representing the ion accumulation as a function of externally applied frequency at different initial bulk concentration. The ion concentration gradient is increasing with the potential in high linearity up to $500 \mathrm{mM}$ electrolyte concentration and rms potential up to $2.0 \mathrm{~V}$. Such result demonstrated good agreement with the equivalent $\mathrm{RC}$ circuit model in the way that high rms potential gives more charge within the capacitance ability of the capacitance of the electric double layer. Also, it can be observed that the frequency dependence is majorly dominating in low frequency region due to the surface capacitance of electric double layer and will be much lower as the frequency increases. 


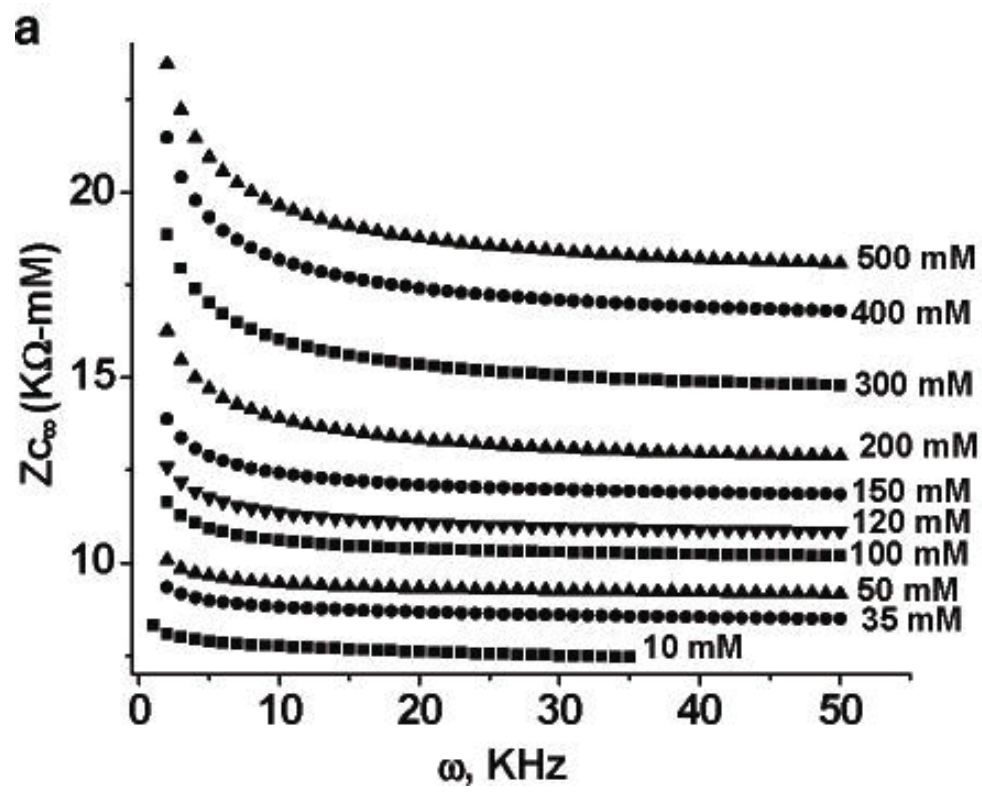

Figure 12: Weak frequency dependence of impedance to frequency [82] (Reproduced with permission)

Theoretical studies on non-Faradaic process under AC potential has been developed recently[83-88]. Steric effects were proposed to break down the traditional assumption of infinitely small ion size[89, 90]. Additional calculations were also performed at large potentials beyond the linear regime. As a result, the known electrical double layer was gradually substituted by a model containing 3 layers including a compacted layer, middle layer (diffuse layer) and a third diffusion layer between the traditional electrical double layer and the bulk solution [91].

Direct flow table for cation flux behavior within one complete AC period has been published by Suh et al. [92] using the asymptotic analysis in nonlinear regime. They proposed that the ion concentration development with time falls into a strong nonharmonic pattern. In middle layer, both cation and anion concentrations change at same magnitude and angular phase thus such 
layer is regarded as a buffer layer between bulk electrolyte and inner layer. The outer layer then is the diffusion layer which function as a diffusion layer of excess salt concentration and is vanishes once system gets into quasiequilibrium state.

\subsubsection{Non-Faraday Processes under AC Electric Potential in Bulk Electrolyte}

Despite the development of research on non-Faradaic processes on electrode-electrolyte interface, general assumptions of constant solution properties are often made for bulk electrolyte under AC electric fields, especially at high frequencies.

Such assumption could be only valid in ideal conditions as the work performed by Zhang et al. [93], where they made the assumptions of thin dielectric layer, small potential, no steric effect and symmetrical electrolyte.

Simulation results from their work indicate that the electrolyte solution only oscillates with applied potential below $100 \mathrm{kHz}$ and tends to be stabilized beyond $10 \mathrm{MHz}$. They also mentioned that under such ideal condition, even under low frequencies, the ion oscillations are symmetric and inducing zero change in time averaged solution condition.

However, in more realistic condition [83, 84, 88-90, 94], Olesen et al. [85] suggested a salt concentration change even in bulk solution under AC electric field. They suggested under the condition of large potential and thick electrical double layer, the potential drop across bulk electrolyte demonstrates strong 
non-linear behavior. The claimed that despite the oscillating nature of the concentration curve, the real concentration stayed above zero at all time, which indicates a time averaged electrolyte concentration increase in the inner edge of diffusion layer, resulting in a depletion of electrolyte in the bulk area due to the mass conservation.

They attributed such concentration depletion to the non-symmetrical expelling and adsorbing of ions from or into diffuse layer. They claimed that the expelling and adsorbing processes both exist at lower potentials so the net salt adsorption thus bulk concentration depletion was low. However, at higher potential, the adsorbing process dominated over the expelling process due to the limited number of coions which resulted in strong concentration depletion in bulk electrolyte in the system due to the induced non-symmetrical expelling and adsorbing processes of ions.

However, experimental verification to ion/electrolyte concentration change under AC electric field was very limited, especially for non-Faradaic processes.

Yossifon and Chang [95] examined the extended space charge layer (SCL) beyond the Debye layer under very low frequency at $0.1-1 \mathrm{~Hz}$ AC signal. The research on the SCL was initiated by the observation of the I-V characteristic of ion-selective membranes showing current density exceeds the limiting current density at enough high voltages. Yossifon et al. applied a straight nanoslot to model the ion-selective membrane condition. Although the AC electrokinetics is not the major target in this work, both of the ion enrichment 
and depletion at the each ends of half period of alternating field in the linear nanoslot were observed and demonstrated strong frequency dependency. An observation of decrease in magnitude of ion depletion and enrichment with increase of frequency was made from 0.001 to $1 \mathrm{~Hz}$ and no ion depletion or enrichment was found beyond $1 \mathrm{~Hz}$.

Ion enrichment was also observed in nanochannels systems [92, 96-107]. One of the most interesting and unique effect is the electrical double layer overlapping in normal microchannels. Within micro-scaled channels, the Debye length (electrical double layer thickness) is much smaller than the channel width. Majority electric potential drop and ion concentration gradient thus also exist within the electrical double layer, resulting non-zero potential and neutral charge in bulk solution.

However, in nanochannels [103], when the channel widths decreases to nanometer scale, the Debye length can sometimes be larger than the channel width, especially in dilute solutions where the electrical double layer has higher thickness. Thus, the two electrical double layers built on the walls at both sides of the nanochannels extend to the 'bulk' part of the nanochannels and overlap with each other. Such electrical double layer overlapping would result in the electric potential in the bulk area being influenced by the surface charging condition and also the much higher concentration of the counterions and much lower concentration of coions, with respect to the surface charging condition. 
a

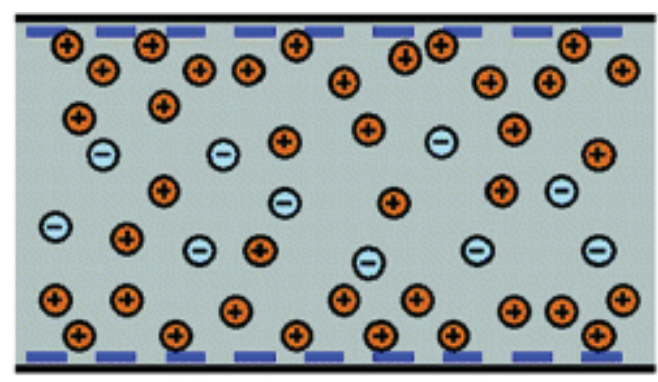

Microchannel

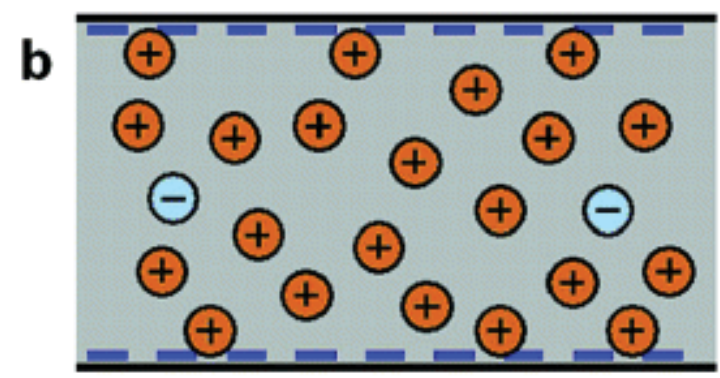

Nanochannel

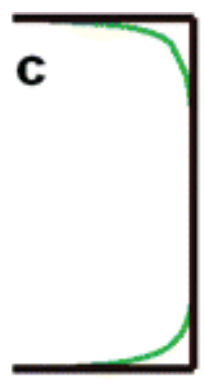

Electric Potential

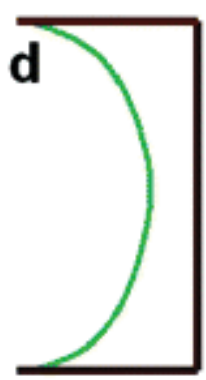

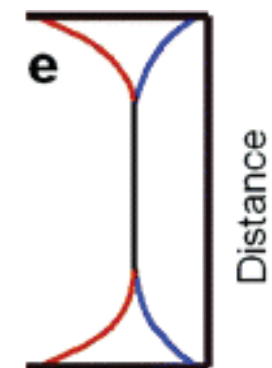

Ionic Concentration

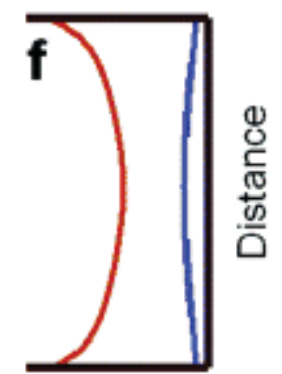

Figure 13: Comparison of electric potential $(c, d)$ and ionic concentration $(e, f)$ distribution in (a) microchannel and (b) nanochannels [103] (Reproduced with permission)

It would be reasonably expected that even under AC condition, especially at the frequencies below electrode charging frequency, such electrical double layer overlapping phenomena would still exist and the potential drop and ionic concentration distributions in bulk areas in nanochannels would be still affected by surface charging condition.

\subsection{Conclusions}

Experimental results in AC electrokinetics microfluidics systems including EP, DEP, ACET and ACEO are highly dependent on medium solution properties. Such solution properties such as ionic concentration, ionic strength, $\mathrm{pH}$ value and conductivity are normally assumed to be constants 
under AC electric field. However, it has been previously demonstrated that both Faradaic and non-Faradaic processes affect system properties.

AC Faradaic processes have been thoroughly studied during 1910 to 1950 s. In recent years, solution property change in ACEO microfluidics systems induced by AC Faradaic process has also been examined under parallel electrodes array systems. Both theoretical and experimental works have been reported on multiple dependencies of AC Faradaic processes including initial electrolyte concentration, applied electrical potential, applied frequencies, electrode material and time dependence. In short summary, the $\mathrm{AC}$ faradaic reaction rate increase with electrolyte initial concentration, applied electric potential and decrease with applied frequency and reaction time.

AC non-Faradaic processes, however, have not been studied as AC Faradaic processes. Most published (both experimental and theoretical) works on this topic have been focused on the ion/electrolyte $\mathrm{AC}$ transport within the electrical double layer region (or, according to more advanced theory, electrical 'triple' layer region). However, only few works have been published regarding AC non-Faradaic process in bulk electrolyte. Some experimental works have only accomplished at very low frequencies. Other experimental works have completed within nanochannels in nanometer scale instead of the micrometer scales solution normally applied in microfluidics systems. Theoretical works have predicted bulk concentration change induced by neutral salt adsorption by the electrical double layer. However, those works focused on spatially uniform electric field between planar electrodes. 
No substantial studies of AC non-Faradaic process in spatially non-uniform electric field have been found in the literature, especially from experimental aspect. Thus, the potential variation of solution properties changes under non-uniform AC electric field besides of electrochemical reaction has not been considered for the $\mathrm{AC}$ electrokinetics microfluidics systems. As a result, such microfluidics systems are facing challenges in terms of biocompatibility, accuracy as well as design validity. To decrease the potential system failure in $\mathrm{AC}$ electrokinetics microfluidics induced by non-Faradaic process, the behavior of ionic concentration, a crucial solution property affecting ionic strength, conductivity and permittivity, must be investigated. 


\section{CHAPTER 3. SPATIALLY-VARIANT RED BLOOD CELL CRENATION IN AC NON-UNIFORM FIELDS[108 $]^{1}$}

\subsection{Introduction}

Microdevices, also called lab-on-a-chip (LOC) and micro-Total Analysis System ( $\mu$ TAS) have shown great potential in quantitative chemical analysis such as single cell impedance measurements [109], cell/particle concentration [110] [111], separation [112] [113], manipulation [30-32] in addition to other mechanical, biological or medical diagnostic applications. Advantages are small volume samples, short analysis time, and high efficiency [32]. Disadvantages are reproducibility between cell populations, device reliability exceeding macro-scale analytical techniques, and the bulkiness of chip support systems. This work has implications in the reproducibility and reliability area.

Prior work has investigated electrolytic manipulation including electrophoresis, electroosmosis, electrothermal, and dielectrophoresis of red blood cells in direct-current (DC) [28] and alternating-current (AC) [31] [30] devices. Cell crenation was observed during long-term DEP pearl

1 This chapter is a reproduction of an article which was published on Biomicrofluidics in Mar 2014.

Biomicrofluidics, 2014. 8(2)

DOI: $10.1063 / 1.4867557$

Reproduced by permission of The Royal Society of Chemistry 
chain formation and transition (over $60 \mathrm{~s}$ ) at length scales above $10 \mu \mathrm{m}$ [31]. Thus, this work systematically explores the physical mechanisms inducing the previously observed, but not quantified cell crenation.

Without electric fields present, cell deformation can occur due to abnormal cell physiology or chemical abnormalities [114], medium ionic strength [115], mechanical forces [116], medium $\mathrm{pH}$ [117], absolute temperature value [118], and rapid temperature changes [119]. For healthy red blood cells, medium ionic strength, or tonicity, is the most prevalent cause for cell crenation. Osmotic pressure is the osmolyte balance between medium solution and the interior of cells. Cell semipermeable membranes have ion and water channels such that water and ion content are in quasi-steady state between intra and extracellular regions. As a result, cells will absorb water to swell/hemolysis (e.g. ghosting), stay normal/healthy, or lose water to dehydrate/shrink (e.g. crenate) at medium ion concentrations that are lower than, equal to, or higher than intracellular ion concentration, respectively. In the presence of electric fields, cell electroporation and electrofusion have been observed [120].

In our system, experiments demonstrate cell crenation in non-uniform AC fields with a physiological ionic buffer. Carefully constructed control experiments elucidate that $\mathrm{pH}$, temperature, rapid temperature change, and electroporation are not responsible for cell deformation in our system. Physiologically relevant conductivities $(1000 \mu \mathrm{S} / \mathrm{cm})$ and frequencies greater than $250 \mathrm{kHz}$ are utilized to avoid $\mathrm{AC}$ electroosmosis (ACEO). AC 
electrothermal (ACET) flows are quantified and do not explain the observed phenomenon. Results support that cell crenation occurs because of an osmotic imbalance generated by the formation of ion concentration gradients likely induced by an ion flux described by the following form of the Nernst-Planck equation:

$$
J_{i}=-z_{i} u_{i} F c_{i} \nabla \varphi-D_{i} \nabla c_{i}+c_{i} v+R_{i},
$$

where, for species $i N_{i}$ is total ion flux, $z_{i}$ is charge valence, $u_{i}$ is ion mobility, $c_{i}$ is ion concentration, $\varphi$ is electric potential, $D_{i}$ is diffusivity, $v$ is convective flux velocity and $R_{i}$ is reaction rate. The physical reasons for a bulk ion concentration gradient formation is discussed with respect to electric migration (first term), countered by diffusion (second term), fluidic flow (third term), and Faradaic currents (fourth term).

$\mathrm{RBC}$ crenation, which is observed at longer length and time scales, is introduced in section 3.2. In section 3.3, experiments are discussed that examine potential and peak-to-peak dependency of the RBC crenation. Optically observed 2D cell area changes are quantified to track cell deformation. In section 3.4, control experiments are discussed that examine cell healthiness, electroporation, electric field induced flows, dielectrophoresis (DEP) forces, temperature, and medium $\mathrm{pH}$. We conclude that the observed cell crenation is due to an osmotic pressure change induced by an ion concentration increase in the bulk fluid at longer time scales. These experimental results challenge the assumption that fluid within nonuniform $\mathrm{AC}$ microfluidic systems have constant uniform ion concentrations at distances larger than the double-layer thickness. Further implications of these observed results are that induced ion propagation can 
influence local electrical conductivity, conductivity, density and other fluid properties thus inducing unexpected fluid behaviors and cell/particle responses. 


\subsection{Materials and Methods}

\subsubsection{Device Fabrication}

Microdevices were custom fabricated with a $100 \mu \mathrm{m}$ gap between perpendicular Ti/Au electrodes and overlaid by a $900 \mu \mathrm{m}$ by $800 \mu \mathrm{m}$ and $70 \mu \mathrm{m}$ high PDMS fluidic chamber [31]. Standard soft photolithography fabrication procedures (EVG 620 Mask Aligner) were followed [30] for both a) Ti/Au electrodeposition (Perkin-Elmer 2400 sputter tool) to a depth of $200 \mathrm{~nm}$ and b) fluidic layer relief masters. Fig. 14 shows a) a photo of the entire device, b) a cartooned view of the device layout with electrodes in yellow and fluidic chambers and channels in blue and c) magnified view of the experimental test chamber with the microscope viewing area outlined in red.

\subsubsection{Materials}

Human blood samples from volunteer donors were obtained by certified phlebotomists. Whole blood was stored at $4{ }^{\circ} \mathrm{C}$ within $10 \mathrm{~min}$ after donation. Experiments were completed within 2 days to avoid cell membrane phospholipid degradation [30]. Red blood cells were separated from plasma by centrifugation at $110 \mathrm{rcf}$ for $5 \mathrm{~min}$. Plasma was removed and $0.9 \% \mathrm{NaCl}$ was added and centrifugal washing was repeated twice. 
a)

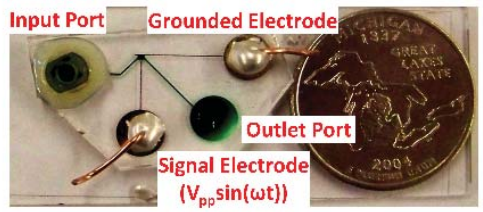

b)

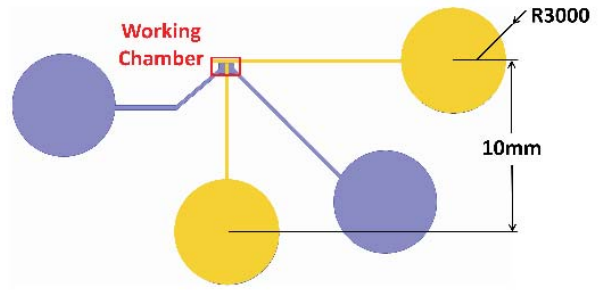

c)

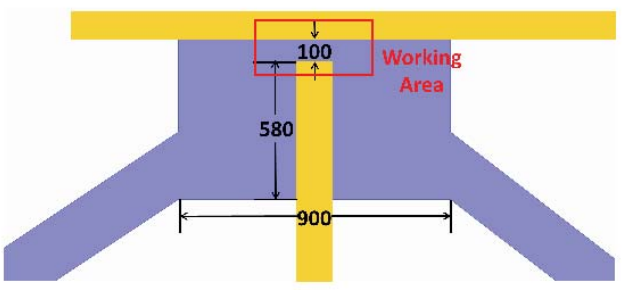

Figure 14: Microdevice configuration. a): Photo of device, with fluid channel filled with green dye; $b$ ): Device design with dimensions shown, red boxes the working fluid chamber and c): Dimensions for red box in b), and red box is the area covered under $63 \mathrm{x}$ microscope. Units for all numbers are micrometers.

$\mathrm{NaCl}$ ( $>99 \%$ pure, Macron Chemicals, USA) solutions were used at $0.7 \%$ (hypotonic, $120 \mathrm{mM}, 1.15 \pm 0.05 \mathrm{~S} / \mathrm{m}$ ), $0.9 \%$ (isotonic, 154 $\mathrm{mM}, 1.5 \pm 0.1 \mathrm{~S} / \mathrm{m}$ ), and $4 \%$ (hypertonic, $685 \mathrm{mM}, 3.6 \pm 0.2 \mathrm{~S} / \mathrm{m}$ ). $\mathrm{NaCl}$ was utilized because the hydrated cation (360 pm) and anion (330 pm) are similar in size with opposite, yet equal charges. Solution $\mathrm{pH}$ was adjusted between 6.9 and 7.1 using $1 \mathrm{M} \mathrm{NaOH}$ (Sigma Aldrich, USA) and $1 \mathrm{M} \mathrm{HCl}$ (Sigma Aldrich, USA). Isotonic solution was utilized as the medium for all experiments except for the controls noted. Dextrose $(>99 \%$, Fisher Scientific, USA) was utilized to maintain 
medium tonicity while alternating initial ionic concentration and conductivity $(0.09 \% \mathrm{NaCl}, 4.5 \%$ dextrose was $0.145 \pm 0.005 \mathrm{~S} / \mathrm{m})$.

\subsubsection{Red blood cell experiments}

Dilutions of 1:400 red blood cells to isotonic $\mathrm{NaCl}$ solution were loaded into the device via the ports seen in Figure 14a). The device was mounted on the microscope stage and pressure between ports allowed to equilibrate for 15 min. AC signals ranging in amplitude of 10, 12.5, 15, and $17.5 \mathrm{Vpp}$ and frequency of $250 \mathrm{kHz}, 500 \mathrm{kHz}, 750 \mathrm{kHz}$, and $1 \mathrm{MHz}$ were applied via an Agilent 33250A waveform generator across the point electrode $\left(\mathrm{V}_{\mathrm{pp}} \sin (\omega \mathrm{t})\right.$ ) and the flat electrode (grounded). Microscopy videos at $63 \times$ magnification were recorded at $1 \mathrm{fps}$ for 10 min with the first $5 \mathrm{~s}$ without applied field to confirm cell healthiness. Each experiment was repeated three or more times.

To ascertain the effects of field gradient and potential AC electroporation as well as temperature and $\mathrm{pH}$ impacts on cell crenation, $100 \mu \mathrm{m}$ gap parallel electrodes at $500 \mathrm{kHz}$ and $15 \mathrm{~V}_{\mathrm{pp}}$ were compared to the perpendicular electrode results. Medium conditions from $0.9 \% \mathrm{NaCl}$ to $0.09 \% \mathrm{NaCl} / 4.5 \%$ dextrose were used with both perpendicular and parallel electrode devices to explore electroporation impact on cell crenation.

\subsubsection{Flow Measurements}

ACEO and ACET flows, when present within the device and dominating DEP phenomena [121], were quantified by position/time 
tracking of $1 \mu \mathrm{m}$ fluorescent latex beads (excitation $\sim 470 \mathrm{~nm}$, emission $\sim 505 \mathrm{~nm}$, Sigma Aldrich) mixed 1:130 with $0.9 \%$ NaCl. $63 \times$ magnification video was recorded at $3 \mathrm{fps}$.

\subsubsection{Data Analysis}

Red blood cell areas were measured using edge detection software (Zeiss AxioVision 4.8) to identify cell perimeter and thus calculate 2D area. For a single cell, measurement error for $\mathrm{n} \geq 5$ analysis attempts was within 3\%. Measured cell areas for 200 to 300 cells in each of 3 separate experiments were compiled into histograms and the average change in cell area percentage was obtained from the following equation:

$$
\Delta A_{\text {cell }} \%=\frac{\overline{A_{t}}-\overline{A_{20}}}{\overline{A_{20}}} \times 100
$$

where, $\overline{A_{t}}$ and $\overline{A_{20}}$ are average cell areas at times $\mathrm{t}$ and $20 \mathrm{~s}$. Error bars were determined as the standard error of one standard deviation from the mean.

ACET flow velocities were obtained via particle tracing. Curves following particle streak lines were drawn with Zeiss AxioVision software assistance. Path length of the curve per frame at a rate of 3 fps enabled the flow velocity to be calculated via path length/(number of frames $/ 3 \mathrm{fps})$.

COMSOL Multiphysics (Burlington, MA) was utilized to map 2D electric field distribution and ion motion. Microdevice geometry 
shown in Figure 14 was duplicated within COMSOL with No Flux boundary conditions set for the PDMS walls and Electric Potential boundary conditions set for the electrodes $\left(V_{p p} \sin (\omega \mathrm{t})\right.$ for the vertical electrode and 0 for the horizontal electrode, where $V_{p p}=15 \mathrm{~V}$ and $\omega=2 \pi \mathrm{f}=3.14 \times 10^{6}$ at $500 \mathrm{kHz}$ ). Electrostatics physics employing the Poisson equation was utilized within the AC/DC Module. The Nernst-Planck equation was utilized within the Chemical Reaction Engineering Module to obtain ion concentration behaviors over the first 100 periods. Also, solution properties were set according to $0.9 \%$ $\mathrm{NaCl}(0.145 \mathrm{M}$, relative permittivity=77.15)

\subsection{Results and discussion}

Control experiments were first completed to qualify red blood cell size and shape in hypo-, iso-, and hyper-tonicity solutions. Next, isotonic red blood cell areas were measured before and during nonuniform AC field experiments; cell crenation was compiled with time and spatial position in the electric field at each fixed signal frequency and amplitude. Competing effects were systematically explored and included electrothermal flow, temperature changes, $\mathrm{pH}$ and electric field.

\subsubsection{RBCs in Controlled Tonicity Media}

RBCs in $0.7 \%, 0.9 \%$ and $4 \% \mathrm{NaCl}$ with osmotic pressures of 233 , 300, and 1333 Osm (hypo- iso- and hyper-tonic, respectively), were imaged and analyzed as shown in Figures 15 a, b and c. Cells in hypotonic media (2a) gain water changing to a spherical shape that 
equilibrates interior cell osmotic pressure with the lower external osmotic pressure. Cells in isotonic $\mathrm{NaCl}(2 \mathrm{~b})$ remained biconcave in shape. In hypertonic solutions (2c), the red blood cells lose water, shrink and demonstrate the characteristic crenated shape. Two-dimensional cell areas and standard deviations from $\mathrm{n}=5$ experimental repeats at each concentration were calculated and are compiled in Figure 15d. A disadvantage of the 2D analysis is that cells, which swell in 3 dimensions under hypotonic conditions, do not show an appreciably changed 2D area. In hypertonic conditions, decreases in cell area can be a result of either cell shape changes from biconcave to spherical or cell crenation. Increases in 2D cell area can result from a loss of cell water that flattens the biconcave shape making the $2 \mathrm{D}$ area appear to slightly increase. Size polydispersity thus substantially increased for cells in hypertonic conditions due to these differing tonicity crenation thresholds for individual cells (Figure 15c). This is expected in red blood cell populations diverse in cell age and bone marrow growth variations.

\subsubsection{Time Dependence of RBC Crenation in Non-Uniform AC fields}

Washed red blood cells in $0.9 \% \mathrm{NaCl}$ were observed under 10 to $17.5 \mathrm{~V}_{\mathrm{pp}}$, and $250 \mathrm{kHz}$ to $1 \mathrm{MHz}$ sinusoidal $\mathrm{AC}$ signals applied across the perpendicular electrode configuration shown in Figure 14 to create non-uniform electric fields. The original cell area and healthiness was assessed for $5 \mathrm{~s}$ before the field was applied. Red blood cell translational motion (due to DEP) and crenation behaviors were observed for $10 \mathrm{~min}$. 

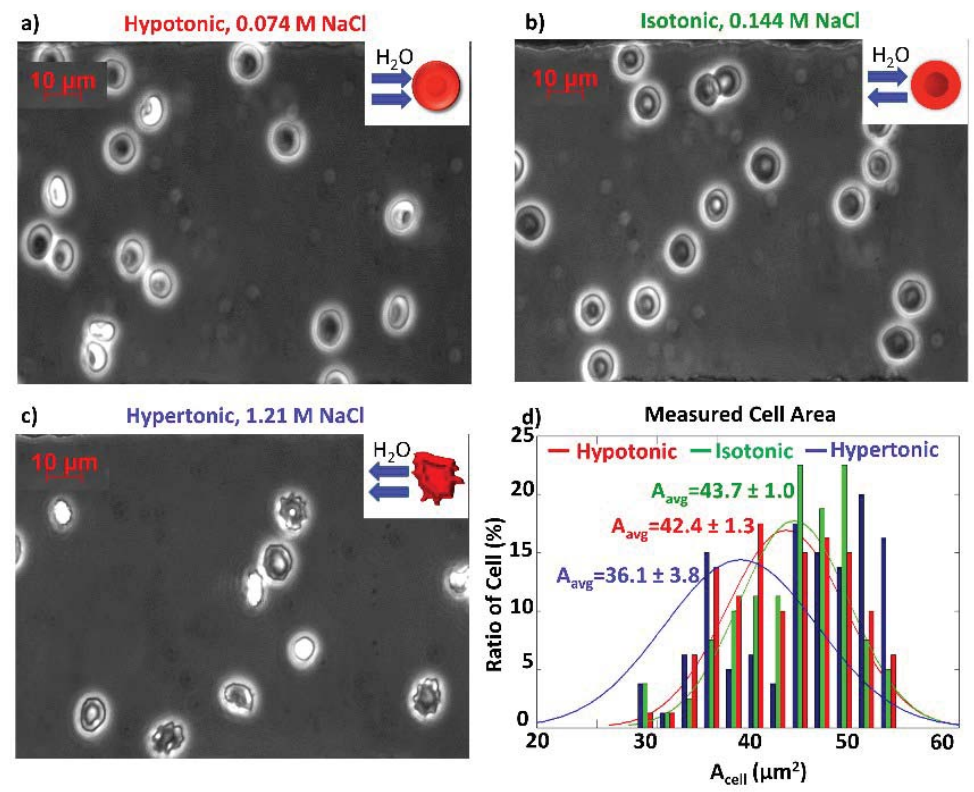

Figure 15: Red blood cells at $63 \mathrm{x}$ in a) Hypotonic $(0.72 \% \mathrm{NaCl})$ b) Isotonic $(0.9 \% \mathrm{NaCl})$ and c) Hypertonic $(7 \% \mathrm{NaCl})$ solutions d) Cell area distribution obtained from image analysis within the : Hypotonic: red; Isotonic: green; Hypertonic solution: blue. Cell deformation and crenation due to the hypertonic solution are observed and reflected in area analysis 

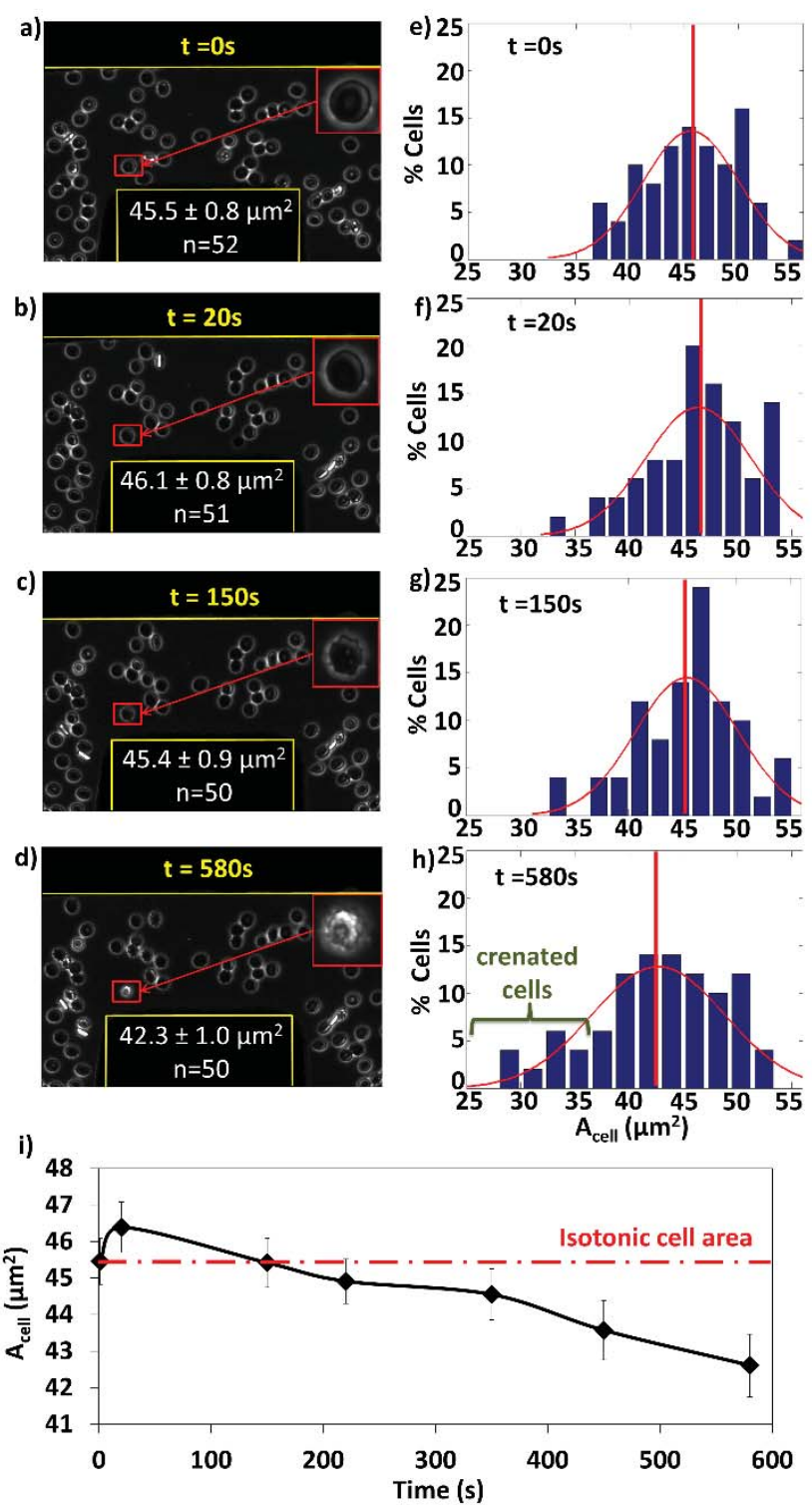

Figure 16: Time series of experiment at $500 \mathrm{kHz} 15 \mathrm{~V}$ peak-to-peak potential at $\mathrm{t}=0 \mathrm{~s}$ to $\mathrm{t}=580 \mathrm{~s}$ (a-d); histogram plot for cell area distribution at corresponding time point (e-h), and $\mathrm{i}$ is the cell average area change with time, with error bars shows standard deviation of cell sizes. Cells started to crenate in figure c) and crenation at higher level and higher amount in d). i) shows that cell area decrease with increase of time

Microscopic images at $63 \times$ of the video region for an applied 
voltage of $15 \mathrm{~V}_{\mathrm{pp}}$ and $500 \mathrm{kHz}$ are shown in Fig. 16 at a) $\mathrm{t}=0 \mathrm{~s}$, c) $20 \mathrm{~s}$, e) $150 \mathrm{~s}$, and g) $580 \mathrm{~s}$, each with an enlarged single cell in the inset. Cell areas at each time were compiled into histograms with the size distribution curve overlaid and average shown by the vertical line as shown in Fig. $16 \mathrm{~b}, \mathrm{~d}, \mathrm{f}$ and $\mathrm{h}$. The progression of the average cell area over time is shown in Fig. 16i. Cells first align and roll due to the DEP force while maintaining their biconcave shape (Fig. 16 a through d). However, beginning at $150 \mathrm{~s}$ (Fig. 16e) at distances greater than 30 $\mu \mathrm{m}$ from the electrodes, cells progressively shrink and crenate as demonstrated by inset cell images in Fig $16 \mathrm{e}$ and $\mathrm{g}$ as well as the corresponding histograms showing the increased number of cells below $35 \mu \mathrm{m}^{2}$. The histograms illustrate the decline in number of cells in the mid range from 40 to $50 \mu \mathrm{m}^{2}$ and an increase in the number of cells at the smaller crenated size and a small increase above $50 \mu \mathrm{m}^{2}$; combined, this generates a shift to smaller mean cell size area (Fig. 16i) and an increase in polydispersity as was seen in Figure $3.2 \mathrm{c}$ and $\mathrm{d}$ for hypertonic media conditions.

\subsubsection{Peak-to-peak Potential and Frequency Dependence}

The peak-to-peak potential and frequency dependence of cell crenation at $\mathrm{t}=580 \mathrm{~s}$ was quantified for $10.0,12.5,15.0$, and $17.5 \mathrm{~V}_{\mathrm{pp}}$ with corresponding electric fields of $0.100,0.125,0.150$, and 0.175 $\mathrm{V} / \mu \mathrm{m}$ at $250 \mathrm{kHz}, 500 \mathrm{kHz}, 750 \mathrm{kHz}$, and $1.00 \mathrm{MHz}$ as illustrated in Fig. 17. Cell area changes were calculated according to equation 1 with standard deviations calculated from $n \geq 3$ experiments. 

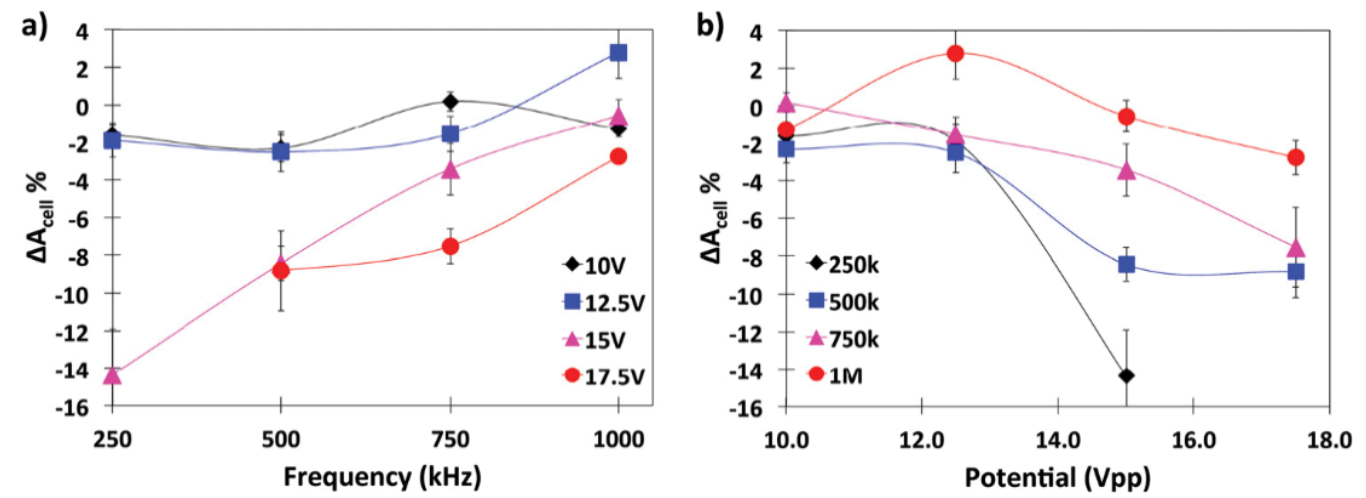

Figure 17: Frequency and potential dependencya) frequency dependency of cell shrinkage percentage at $10 \mathrm{~V}$ (blue), $12.5 \mathrm{~V}$ (red), $15 \mathrm{~V}$ (green) and $17.5 \mathrm{~V}$ (purple); b): potential dependency of cell shrinkage percentage at $250 \mathrm{kHz}$ (red), $500 \mathrm{kHz}$ (blue), $750 \mathrm{kHz}$ (green) and $1 \mathrm{MHz}$ (purple). This shows cell deformation intensity increase with peak-to-peak potential and decrease with frequency, which implies the ion propagation increase with peak-to-peak potential and decrease with frequency.

Fig. 17a demonstrates the potential dependency of cell crenation from $10.0 \mathrm{~V}_{\mathrm{pp}}$ to $17.5 \mathrm{~V}_{\mathrm{pp}}$ at frequencies from $250 \mathrm{kHz}$ to $1.00 \mathrm{MHz}$. At 15.0 and $17.5 \mathrm{~V}_{\mathrm{pp}}$ for all frequencies, cell crenation increased with decreasing frequency with a maximum measured area decrease of $13 \%$ for $250 \mathrm{kHz}$ and statistically negligible at $1.00 \mathrm{MHz}$. As shown in Fig. $17 \mathrm{~b}$, cell crenation increased (more negative $\Delta \mathrm{A}_{\text {cell }} \%$ ) with increasing electric field strength. At frequencies approaching $1.00 \mathrm{MHz}$, flow recirculation interfered with the $\mathrm{V}_{\mathrm{pp}}$ trends. At $250 \mathrm{kHz}$ and potentials at or above $17.5 \mathrm{~V}_{\mathrm{pp}}$, electrolysis reactions occurred at the electrodes and interfered with cell observation. Fig. 17 illustrates that at $10.0 \mathrm{~V}_{\mathrm{pp}}$, cell areas were nearly constant at all frequencies and at $12.5 \mathrm{~V}_{\mathrm{pp}}$, cell areas increased slightly with field application. Error bars illustrate 
these changes are not statistically significant. However, at frequencies beyond $500 \mathrm{kHz}$ at the highest potentials, the effect of $\mathrm{AC}$ electrothermal flow (ACET) became more prominent as discussed in section 3.4.4 and interfered with crenation behaviors.

\subsubsection{Quantification of Potentially Competing Mechanisms}

Red blood cells have been observed in prior work to exhibit crenation changes induced by electroporation [120], medium solution $\mathrm{pH}$ [117], temperature value [118], and rapid temperature changes [119]. Thus, controls were conducted for each of these factors. To avoid cell health factors, experiments were only conducted on visually healthy cells with average areas between 31.93 and $51.73 \mu \mathrm{m}^{2}$, which is consistent with published normal 2D cell area ranges of 30.17 to 52.78 $\mu \mathrm{m}^{2}[122]$.

\subsubsection{Impact from Electric Potential and Field}

\section{Electric field impact on cell membrane}

Electric fields can impact cell shape via dielectrophoretically-driven alignment with the field (i.e. cell elongation) and electroporation yielding either reversible or irreversible membrane pores. The former, DEP, has a dielectric relaxation timescale on the order of microseconds for red blood cells in this frequency range [35] and is a rapid response force causing realignment and cell rolling along the field lines. This effect is observed in Fig. 16f at $20 \mathrm{~s}$ and does not account for the crenation observed at longer time scales $(t>150 \mathrm{~s})$. The later, electroporation, can cause crenation-like cell shape changes 
similar to our results demonstrated in Fig. 16 [120]. Electroporation is due to electric field induced membrane perturbations that cause nanopores to form and ease ion/water exchange, thus resulting in cell dehydration, crenation and ghosting [123].

In order to test whether electroporation was responsible for crenation, the same red blood cell solution and field conditions were tested, as shown in Fig. 22, in a perpendicular (first column) and parallel electrode (second column) configuration. Electroporation depends upon field strength, but not field gradients [124]. Comparison of images at $580 \mathrm{~s}$ in Fig. $22 \mathrm{~g}$ and $\mathrm{h}$, as well as the corresponding cell area size distributions in Fig. $22 \mathrm{j}$ and k illustrates that crenation was not observed in the parallel electrode configuration at the same field and medium conditions as in the non-uniform case.

Further, in the literature, no evidence of red blood cell electroporation has been reported for our conditions between 10.0 to $17.5 \mathrm{~V}_{\mathrm{pp}}$ or $250 \mathrm{kHz}$ to $1.00 \mathrm{MHz}$ frequency. Electroporation on red blood cells has been observed in a) $100 \mathrm{kHz}$ sinusoidal frequency and $4-5 \mathrm{kV} / \mathrm{cm}$ potentials [124], b) 0.02 to $0.08 \mathrm{~ms}$ pulse signals at $4-5$ $\mathrm{kV} / \mathrm{cm}$ [118], and c) 100, 200 and $300 \mathrm{~ms}$ pulse signal at 1-1.6 kV/cm [125]. Moroz et al. [126] reported electroporation using a similar $0.9 \%$ $\mathrm{NaCl}$ medium with the application of $9 \mathrm{~ms}$ pulse signal at $1.7 \mathrm{kV} / \mathrm{cm}$. Further, electroporation is highly dependent on signal duration [127], decreasing at higher frequencies and shorter signal durations. Our work used much smaller sinusoidal signals of 0.710 to $1.24 \mathrm{kV}_{\mathrm{rms}} / \mathrm{cm}$ and 
much shorter frequency perturbations than are insufficient for electroporation.
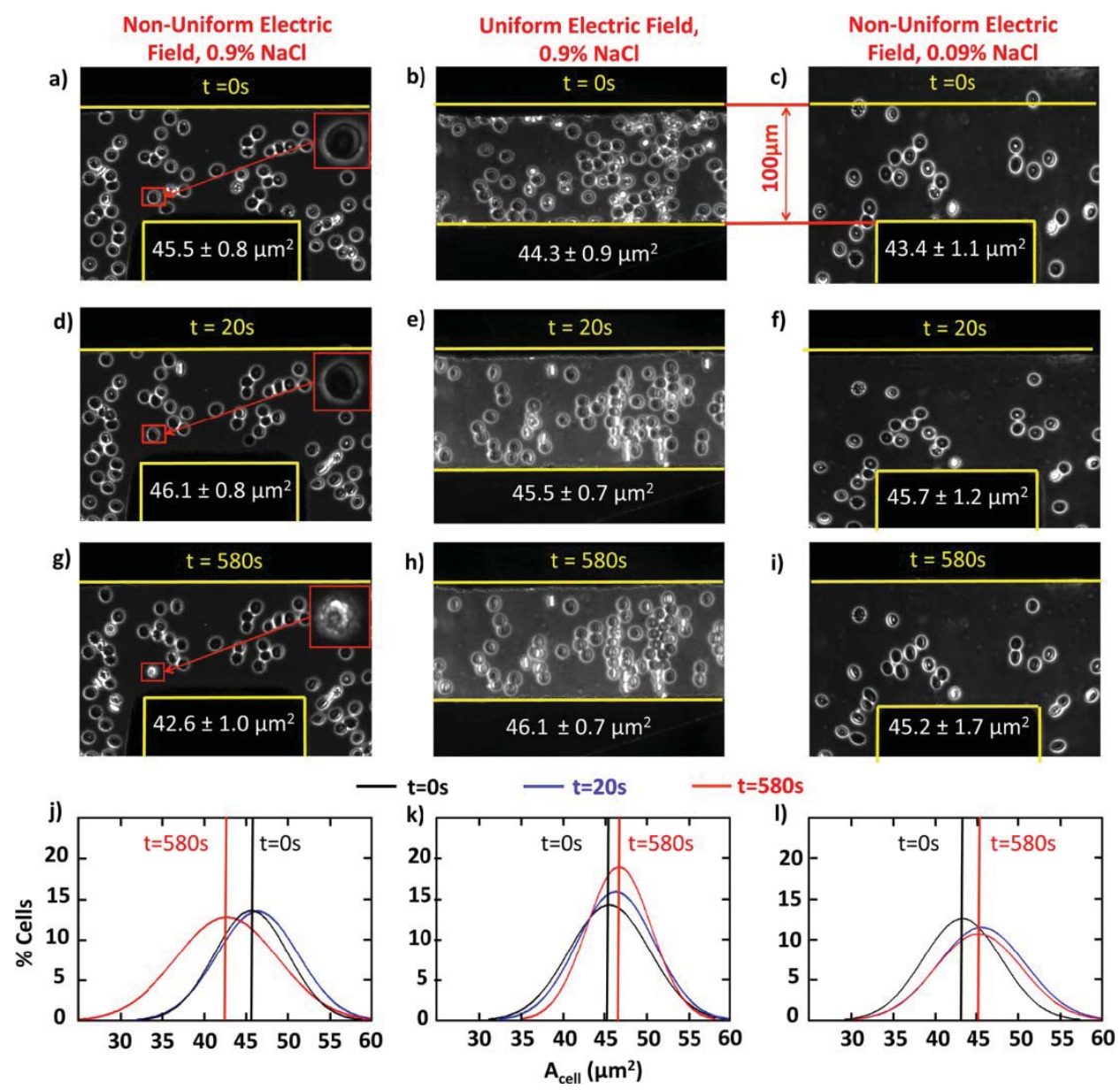

Figure 18: Comparison experiments under 500 kHz, 15Vpp; First

column are $\mathrm{t}=0,20$ and 580 s of experiments in uniform electric field; Second column are same time of experiments using medium solution contain $0.09 \% \mathrm{NaCl}$ and $4.5 \%$ dextrose. Third column are histograms of $\mathrm{t}=0,20$ and $580 \mathrm{~s}$ for nonuniform electric field, $0.9 \% \mathrm{NaCl}$; uniform electric field, $0.9 \% \mathrm{NaCl}$; and nonuniform electric field, $0.09 \% \mathrm{NaCl}$ and $4.5 \%$ dextrose, respectively. It can be observed that shift in distribution of cell area can be observed in c) while no shrinkage 
observed in $\mathrm{f}$ ) and i)

Electroporation also decreases with increasing conductivity [128] and ion concentration [129] due to the decreasing electroporation current described by [129]:

$$
i_{e p}=\frac{\pi r_{m}^{2} F v_{m}}{h} \sum_{x} \frac{D_{x} z_{x}^{2}\left([x]_{i} \exp \left(z_{x} v_{m}\right)-[x]_{0}\right)}{A \exp \left(z_{x} v_{m}\right)-B}
$$

where $r_{m}$ is pore radius, $F$ is Faraday's constant, $h$ is membrane thickness, $D_{x}$ and $z_{x}$ are diffusion coefficient and valence of free ions, respectively, while $[x]_{i}$ and $[x]_{0}$ are ion concentration inside the cell and outside the cell. A and B are constants.

In order to test the medium conductivity dependence of electroporation effects, comparison experiments were conducted using $0.09 \% \mathrm{NaCl}$ and $4.5 \%$ dextrose at a final conductivity of $0.14 \mathrm{~S} / \mathrm{m}$ as shown in Fig. 18 c, f, i, and 1. Solution osmolarity was maintained with neutrally non-charged dextrose and ionic $\mathrm{NaCl}$, such that lower ionic concentrations $(0.0154 \mathrm{M} \mathrm{NaCl}$ or $90 \%$ fewer ions) had a greater chance of yielded electroporation; no cell electroporation or crenation was observed at the same electric field condition of $500 \mathrm{kHz}$ and $15 \mathrm{~V}_{\mathrm{pp}}$. Cells aligned with the electric field from $\mathrm{t}=0$ to $20 \mathrm{~s}$, but no cell crenation was observed at $580 \mathrm{~s}$ as shown in Fig. 18i and the red cell area distribution in Fig. 181. The neutral non-charged dextrose does not electromigrate, react or subsequently propagate spatially within the electric field; dextrose concentrations remain constant. Thus, Fig. 18 data illustrates that ion presence in solution in addition to non-uniform electric field geometry are both important and necessary for cell 
crenation behavior. Further, the electroporation mechanism does not account for the observed red blood cell crenation.

\section{Solution pH}

Human red blood cells are reported to be morphologically stable within 1 unit change of $\mathrm{pH}$ for neutral 7.0 [117]. In DC microfluidic systems, electrochemical reactions, primarily water electrolysis can create microscale $\mathrm{pH}$ gradients [130]. Threshold electrochemical potentials with respect to a standard hydrogen electrode are $0 \mathrm{~V}$ for reduction reactions at the anode and $1.23 \mathrm{~V}$ for oxidation reactions at the cathode. Changes in $\mathrm{pH}$ have only been reported at very low frequencies $[131,132]$. To the best of our knowledge, the highest frequency reporting $\mathrm{pH}$ altering effects was in a $10 \mathrm{kHz} \mathrm{AC}$ electric field at potentials up to $20 \mathrm{~V}_{\mathrm{pp}}$ [121]. The $\mathrm{pH}$ change was much less than 1 unit and the authors concluded this was negligible given the sensitivity of their fluorophore measurement tool [121]. The lowest frequency examined in our current work was $250 \mathrm{kHz}$ and the maximum potential was $17.5 \mathrm{~V}_{\mathrm{pp}}$. Reaction rate changes inversely with frequency [62] so electrochemical reactions are unlikely to influence medium compositions at higher frequencies.

To experimentally verify whether electrolysis reactions could be a factor in crenation, re-examination of the $100 \mu \mathrm{m}$ perpendicular and parallel electrode experiments in Fig. 18 are insightful. At the same electrolyte solution, $0.9 \% \mathrm{NaCl}$, and electric potential, $15 \mathrm{~V}_{\mathrm{pp}}$, electrochemical reactions are equally probable in both the non-uniform and uniform electric fields. However, no cell crenation was observed 
in the parallel electrode system (second column with $5 \mathrm{~b}, 5 \mathrm{e}$, and $5 \mathrm{~h}$ ) and no significant peak shift was quantifiable in $5 \mathrm{f}$. Thus, $\mathrm{pH}$ shifts due to electrolysis are unlikely and do not account for the crenation observed.

\subsubsection{Absolute Temperature Value and Change Temperature in Media}

The passage of current through conductive liquids raises temperatures via Joule-heating [22]. Both high absolute temperatures and rapid temperature changes have been shown to lead to cell deformation $[118,133,134]$. Although both mechanisms exist in our system, neither of them is significant enough to induce observable cell crenation.

Increases in medium temperatures, $\mathrm{T}$, can create osmotic pressure differences $(\Delta \Pi)$ via the colligative effect induced by the equilibrium between medium and cell cytosol water chemical potential. This mechanism occurs over time-scales on the order of hours $[133,134]$ :

$$
T T P=\Delta T \times t
$$

where TTP is temperature-time product $\left({ }^{\circ} \mathrm{C} \cdot h\right), \Delta T$ is temperature difference $\left({ }^{\circ} \mathrm{C}\right)$ and $\mathrm{t}$ is the operation time $(\mathrm{h})$. To estimate the temperature difference, Joule heating temperature jumps in a parallel electrode system can be estimated via [22]:

$$
\Delta T=\frac{\sigma V_{r m s}^{2}}{8 k}
$$

where for the conditions examined, the maximum root mean square potential is $6.19 V_{r m s}$ aqueous thermal conductivity is $k=0.58 \mathrm{~W} /(\mathrm{mK})$, 
and conductivity is $\sigma=1.5 \mathrm{~S} / \mathrm{m}$, which predicts $\Delta T=12.4^{\circ} \mathrm{C}$. This prediction does not account for thermal dissipation through the glass bottom and the PDMS sides and top. TTPs greater than $48^{\circ} \mathrm{C} \cdot \mathrm{h}$ are required for noticeable cell membrane perturbations [133, 134]. In our system, the maximum possible temperature change noted above is $12.4{ }^{\circ} \mathrm{C}$ and the operation time is 10 minutes leading to TTP $\approx 2{ }^{\circ} \mathrm{C} \cdot \mathrm{h}$, which is insufficient to generate a noticeable osmotic pressure difference, $\Delta \Pi$, or crenation.

Second, rapid temperature jumps of $2{ }^{\circ} \mathrm{C}$ or greater in $2 \mu$ s can induce thermal osmosis, which imbalances chemical potentials causing hemolysis [119]. A $0.01{ }^{\circ} \mathrm{C}$ temperature difference was shown to induce a $1.3 \mathrm{~atm}$ pressure difference yielding crenation [119]. An order of magnitude time, $t_{d i f f}$, estimate to reach thermal equilibrium [22] can be obtained from:

$$
t_{\text {diff }}=\frac{\rho_{m} c_{p} l^{2}}{k}
$$

with medium density $\rho_{m}=1.009 \mathrm{~g} / \mathrm{cm}^{3}$, heat capacity $c_{p}=4.18 \times$ $10^{3} \mathrm{~J} / \mathrm{kg} \cdot \mathrm{K}$, and minimum length scale $l=100 \mu \mathrm{m}$, to yield $t_{\text {diff }}=10^{5} \mu \mathrm{s}$. Thermal hemolysis is induced 3 orders of magnitude slower at $100 \mu$ s [119]. Thus, temperature jumps sufficient to induce cell osmotic pressure shifts are not possible at our conditions within the microdevice.

To experimentally verify whether Joule heating could yield absolute temperatures or rapid temperature jumps leading to crenation, 
the same $100 \mu \mathrm{m}$ gap parallel electrode experiments were conducted with highest ionic condition of $0.9 \% \mathrm{NaCl}$ solution at a $\mathrm{V}_{\text {rms }}$ of 6.19 , which should induce the largest heat generation, and most rapid temperature jump. The data was very similar to Fig. 18 b, e, h, and k which demonstrate no cell crenation. Thus, neither the absolute temperature nor the temperature jump is the mechanism inducing cell deformation.

\subsection{Discussion}

Spatially non-uniform AC fields were shown to induce red blood cell crenation under isotonic conditions with higher medium ion conductivities. Crenation was assessed by quantifying 2D cell area. Crenation was greatest at lower frequencies near $250 \mathrm{kHz}$ and became less pronounced as frequencies approached $1.00 \mathrm{MHz}$. Higher potentials up to $17.5 \mathrm{~V}_{\mathrm{pp}}$ yielded the greatest crenation. None of the competing mechanisms (electroporation, $\mathrm{pH}$, absolute temperature, or temperature jump) showed comparable crenation to the non-uniform electric field results from Fig. 16. As a result, competing mechanisms were found to be insufficient at the medium, electric field and microdevice conditions employed here to account for the crenation observed at $10 \mathrm{~s}$ of $\mu \mathrm{m}$ from the electrode surfaces.

To explain the demonstrated time sequence for cell crenation, the lack of crenation in all uniform field experiments as well as the potential and frequency dependence, our suggested explanation is that local solution ion concentration changes from isotonic to hypertonic via ion electromigration unevenly biased by the spatially 
non-uniformities of the electric field.

\subsubsection{Physical Reason for Ion Concentration Gradient}

In this section, we were trying to propose a feasible mechanism, but future work is required to study this both theoretically and experimentally to further prove of concept. For an ion concentration gradient to form and induce local solution tonicity changes, the ion flux $J_{i}$ must be unbalanced. Ion flux $J_{i}$ depends upon ion electromigration, diffusion, convection, and reaction [135] so ion concentration with time is:

$$
\frac{\partial c_{i}}{\partial t}=-z_{i} u_{i} F \nabla\left(c_{i} \nabla \varphi\right)-D_{i} \nabla^{2} c_{i}+v \cdot \nabla c_{i}+R_{i}
$$

Each ion transport mechanism is discussed separately:

1) The second term on the right hand side of the equation, diffusion, is a passive process driven by concentration gradients and in a simple salt solution with only one cation and one anion $(\mathrm{NaCl})$, this mechanism cannot actively initiate a biased propagation of ions in an initially uniform concentration. Once a concentration gradient is established, diffusion would counter this and push the system to a uniform concentration.

2) Convection is the third term on the right hand side. Electric field induced $\mathrm{AC}$ electroosmosis (ACEO) and $\mathrm{AC}$ electrothermal (ACET) flows are sources of convection in this otherwise stationary system. In our high conductivity and higher frequency experimental 
conditions, ACEO was not observed and can be reasonably neglected. However, ACET was observed. This mechanism is caused by local Joule heating of the fluid under high frequency, high potential conditions. Local heating alters local fluid density, viscosity, conductivity and electric permittivity. The spatially nonuniform fluid properties then induce ACET flow [136, 137]. Fluorescent latex beads $(1 \mu \mathrm{m})$ were used to image and estimate fluid flow as shown in Table 1. The velocity and flow pattern area increased with increasing frequency, verifying that our applied potentials fall in the permittivity dominant regions for ACET flow. The frequency dependencies of ACET velocities in Table 1 and of cell crenation in Fig. 17 are in opposition. Cell crenation becomes less prevalent while ACET becomes more pronounced with increasing frequency. Over small length scales, ACET flows would smooth any developing ion concentration gradients, and thus reduce the prevalence of red blood cell crenation.

Table 1: Frequency dependence of induced ACET flow velocities at $\mathrm{V}_{\mathrm{pp}}=15 \mathrm{~V}$

\begin{tabular}{|c|c|c|c|c|c|}
\hline Frequency $(\mathrm{kHz})$ & 250 & 500 & 750 & 1000 & 10,000 \\
\hline Measured Velocity $(\mu \mathrm{m} / \mathrm{s})$ & 1.7 & 2.2 & 26 & 30 & 60 \\
\hline
\end{tabular}

3) Reactions or Faradaic charging can induce bulk concentration changes which induce ACEO reversal [138] at electrode charging frequencies less than $100 \mathrm{kHz}$ calculated from $\tau_{c}=\lambda_{D} L / D$ [138]. However, experimental conditions presented here are greater than $250 \mathrm{kHz}$ where the reaction rate is not 
significant and the electric double layers cannot be established on electrode surfaces [139]. Further, Fig. 18 comparison experiments using parallel electrodes corroborate this conclusion. Any electrode reactions/Faradaic charging would be the same or even larger for parallel electrodes than for orthogonal electrodes due to slightly greater parallel electrode surface areas. This is not observed. Thus, electrode reactions/Faradaic charging mechanisms are not responsible for the ion concentration gradients formed.

4) Electromigration is an electrical force-induced motion of ions. Fig. 18 illustrated cell crenation in non-uniform fields but not in uniform electric fields. Red blood cells crenate in response to local hypertonicity, which indicates local ion concentration gradients in non-uniform electric fields, but not in uniform electric fields. Thus, we hypothesize that local ion concentration gradients are induced by gradients in the electric field. For the red blood cells, the osmotic pressure change is likely induced by local ion accumulations resulting from unequal oscillating ion migration that is a direct result of the $\mathrm{AC}$ electric field non-uniformity. According to the RC model of the electric double layer, the potential drop across the electric double layer is finite at $>100 \mathrm{kHz}$ as the electrode charging time is $t_{c}=5.6 \times 10^{-5} \mathrm{~s}$ [140] so the electrode is not fully screened and the potential drop across the bulk fluid is not infinitely small. At this quasi-steady state condition, the time average $\left\langle\frac{\partial c_{i}}{\partial t}\right\rangle=0$ in equation 7 results in 
$-z_{i} u_{i} F \nabla\left(c_{i} \nabla \varphi\right)=D_{i} \nabla^{2} c_{i}$ if we neglect the convection term $v \cdot \nabla c_{i}$ and reaction term $R_{i}$ as discussed in $\# 2$ and $\# 3$ above.

Experiments indicate cell crenation is induced in ionic solution by the nonuniformities in $\mathrm{AC}$ electric fields. Based on the term-by-term discussion in \#1 through \#4, one can neglect convective flow, reaction, and ion-ion interactions in a preliminary hypothesis regarding the causes of the red blood cell crenation. Since the fluid is nearly stagnant for much of the frequency/potential space explored, Stokes drag on the small hydrodynamic radius of the ions would be the same everywhere. We speculate herein that cell crenation is caused by ion concentration gradients induced by high frequency, non-uniform electric fields.

Since the lowest frequency we applied was $250 \mathrm{kHz}$, corresponding to $4 \times 10^{-6} s$ for one period, and $5.6 \times 10^{-5} s$ [140] are required for electrode charging time, there is not sufficient time to establish the electric double layer and the electrode is not fully screened. In the presence of an ion concentration gradient, diffusion, $D_{i} \nabla^{2} c_{i}$ would not be constant. The diffusion coefficient for $\mathrm{Na}^{+}$is $\sim 1.33 \times 10^{-9} \mathrm{~m}^{2} \mathrm{~s}^{-1}$ while $\mathrm{Cl}^{-}$is $\sim 2.03 \times 10^{-9} \mathrm{~m}^{2} \mathrm{~s}^{-1}$. Thus, diffusion time is $\sim 8 \mathrm{~s}$ across the $100 \mu \mathrm{m}$ electrode gap, which is also much longer than the $4 \times 10^{-6} \mathrm{~s}$ electric field period. This means that electric field driven ion electromigration can dominate ion motion.

Poisson's equation, $\nabla^{2} \varphi=\frac{\rho}{\varepsilon}$, which relates the potential, $\varphi$, to net charge density, $\rho$, and constant dielectric permittivity, $\varepsilon$, is used to describe systems with significant deviations from electroneutrality [141]. The experimental 
evidence presented suggest that ion migration due to the electric field, and to a lesser degree due to differences between $\mathrm{Na}^{+}$and $\mathrm{Cl}^{-}$diffusivity, accumulates over AC cycles causing deviations from electroneutrality and eventually becoming apparent via cell crenation after roughly $10^{8}$ cycles.

COMSOL's electrostatics module was used to simulate the electric field strength within the microdevice as shown in Fig. 19a at 15V assuming relative permittivity of 77.15 for $0.9 \% \mathrm{NaCl}$. The corresponding electric field gradient is shown in Fig. 19b. Once converged, additional physics was added via the Nernst-Planck module at $500 \mathrm{kHz}$. The traditional electroneutrality assumption was not imposed. Diffusion, convection, and reaction terms were all neglected by assuming $R_{i}=0$ and $v \cdot \nabla c_{i}=0$. Due to the spatially non-uniform electric field, the electric field strength varies as a function of geometric position, which yields a non-zero $-z_{i} u_{i} F \nabla\left(c_{i} \nabla \varphi\right)$ term in equation 7. Fig. 19c shows ion concentration oscillations at a single point 20 $\mu \mathrm{m}$ from the orthogonal electrode (black dot, Fig. 19a) in the nonuniform AC electric field. The net ion flux oscillates with the field such that ion concentration accumulates slowly over the first 100 periods (computational limitation). Generally, results in Fig. 19a and 6b explicitly mapped the electric field strength and the electric field gradient. However, Fig. 19c is used only to conceptually deliver the general concept we were trying to propose and more detailed work should be done to further study this. 


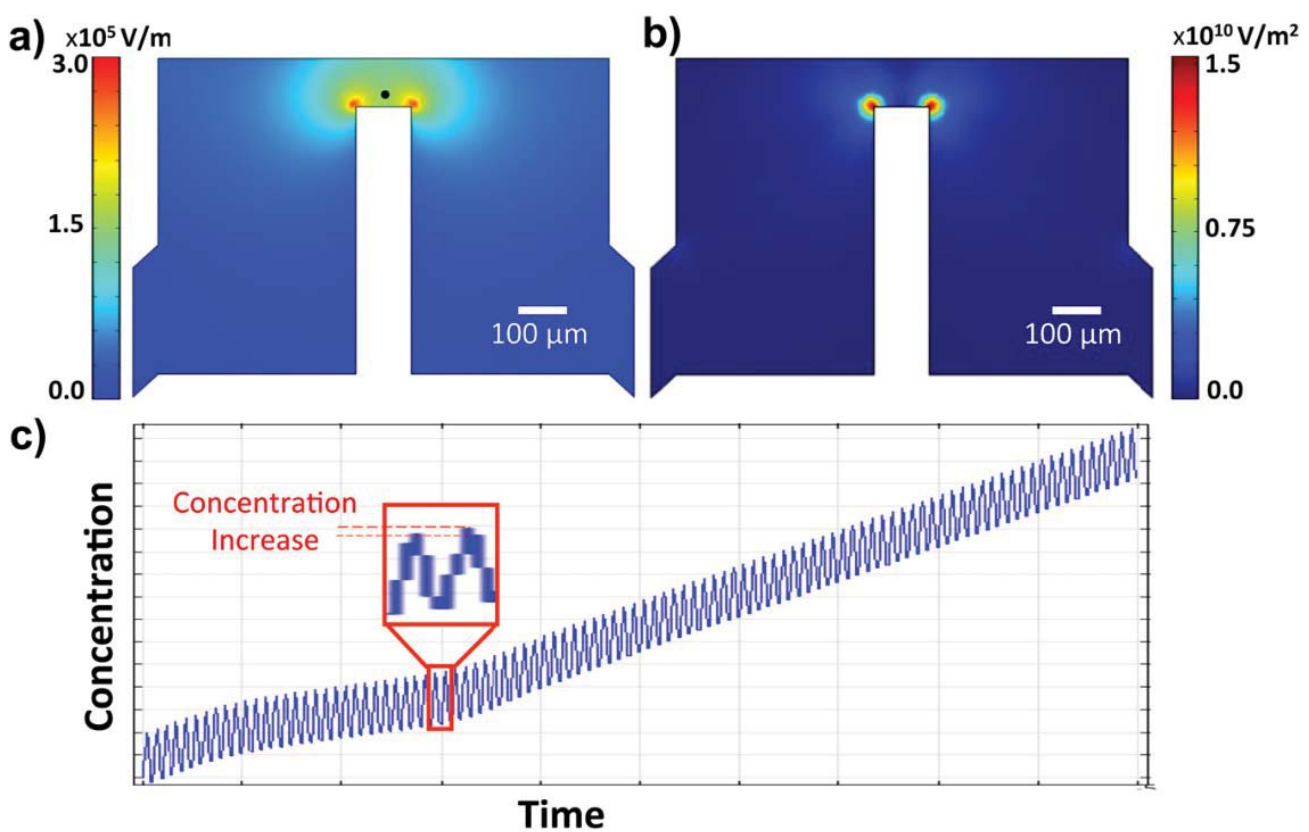

Figure 19: Cell Cell crenation explanation:crenation explanation: (a) Spatial variation of the electric field showing highest electric fields (red) near the vertical electrode. (b) Electrical field gradient showing the highest gradient at the vertical electrode. (c) COMSOL simulation of first 100 cycles of $15 \mathrm{Vpp}$ applied $500 \mathrm{kHz} \mathrm{AC}$ potential showing the oscillation of the ion concentration. Ion concentration increases slightly with each period and accumulates over time.

For the quasi-equilibrium condition, $\frac{\partial c_{i}}{\partial t}=0$, equation 7 can be rewritten as:

$$
D_{i} \nabla^{2} c_{i}=-z_{i} u_{i} F \nabla\left(c_{i} \nabla \varphi\right)
$$

The right hand side of equation 8 is the non-zero electrochemical potential induced by gradient of electric field shown in Fig. 19b. This electric field non-uniformity yields a nonzero $\nabla^{2} c_{i}$ and thus a nonzero ion concentration gradient. Since the diffusion time scale is 6 orders of magnitude slower than ion electromigration due to the electrochemical 
potential term, increases are seen in local ion concentration. This is attributed as the reason that the local osmotic pressure is altered from isotonic to hypertonic thus creating cell crenation.

In the context of this hypothesis, Fig. 18 compared two initially isotonic solutions: the first column is $0.9 \% \mathrm{NaCl}$, while the third column is a mixture of $0.09 \% \mathrm{NaCl}$ and $4.5 \%$ dextrose (nonionic). The applied potential, $\nabla \varphi$, is identical in these experiments, so electromigration for a single ion would be the same $-z_{i} u_{i} F \nabla\left(c_{i} \nabla \varphi\right)$ and so would the counteracting diffusion $D_{i} \nabla^{2} c_{i}$. However, the initial ion concentration is much lower in the $0.09 \% \mathrm{NaCl}$ media, so the induced ion concentration, $c_{i, 0}+\Delta c_{i}$, is not significant enough to induce noticeable cell crenation. While the availability of ions exists in the parallel electrode experiment in column two of Fig. 18, there is no spatial nonuniformity in the electric field so the $-z_{i} u_{i} F \nabla\left(c_{i} \nabla \varphi\right)$ term is net zero and no concentration gradient develops.

Further, the peak-to-peak potential dependence presented in Fig. 17b illustrates that as $\nabla \varphi$ increases, $z_{i}, u_{i}$, and $F$ are constants, and the non-uniformity of electric field causes the ions to accumulate faster over a larger 2D area thus inducting crenation in more red blood cells.

The whole process for ion concentration gradient establishment occurs gradually and thus the osmotic pressure differences between the red blood cell interior and exterior would also change gradually. However, membrane deformation to a crenated state is a threshold phenomenon requiring a sufficient osmotic pressure difference (i.e. tonicity difference) to change. 
This threshold is not identical for all cells in a red blood cell population due to slight morphological and phenotypical differences. The measurable cell crenation observed in Fig. 16 initiates around $150 \mathrm{~s}$ with cell crenation first occurring closer to the high field density signal electrode and progressing up to $\sim 60 \mu \mathrm{m}$ from the electrode edge. The electrical double layer takes $\sim 10^{-4} \mathrm{~s}$ to develop in $0.9 \% \mathrm{NaCl}$ ion concentrations and its length would be $1 \mu \mathrm{m}$ or less. The length and time scales over which crenation was observed are not consistent with an electrical double layer-driven mechanism.

The results compiled in Fig. 17 indicate that greater crenation occurs above $12.5 \mathrm{~V}_{\mathrm{pp}}$ and below $750 \mathrm{kHz}$ frequency. These conditions favor ion concentration establishment and yield larger solution tonicity changes. However, these results are bounded by competing mechanisms. At higher frequencies, electrothermal flows cause mixing, thus preventing sufficient local increases in ion concentration. At lower frequencies, but higher potentials, electrolysis reactions at the electrode surfaces dominate and gas bubbles consume the chamber.

\subsection{Conclusions}

This work experimentally examined the time dependence of cell crenation in a spatially non-uniform AC field. Crenation was observed at distances of 10 to $60 \mu \mathrm{m}$, larger than any possible electrode double layer, starting at time scales over 150 seconds. Potential and frequency dependence were examined and an increase in cell crenation was observed with potential increases and a decrease in crenation was observed with frequency increases. 
The possible physical mechanisms for cell crenation were systematically examined via uniform and non-uniform electric field and high/low ion concentration comparison experiments. Cell deformation due to $\mathrm{pH}$ change, temperature, temperature change, and electroporation were excluded by these comparison experiments and by a term-by-term examination of the ion flux equation. We thus advance the theory that cell crenation is caused by medium tonicity changes occurring via induced electromigration from the electric field gradient, which causes ion concentration gradients in the spatially non-uniform AC electric fields.

These ion concentration gradients occur over large length scales, which are much greater than the electrode double layer Debye length, and at long time scales. This is in contradiction to the general assumption that ion concentration remains spatially uniform at the high frequency $\mathrm{AC}$ fields typically used in dielectrophoretic experiments and contrary to the electroneutrality assumption typically employed for theoretical explorations in these systems. These induced ion concentration gradients will influence local electrical conductivity, density and other fluid properties and may account for anomalous fluid behaviors and particles responses sometimes observed in these microdevices. 


\section{CHAPTER 4. SOLUTION PH CHANG IN NON-UNIFORM AC ELECTRIC FIELDS AT FREQUENCIES ABOVE THE ELECTRODE CHARGING FREQUENCY [142] ${ }^{2}$}

\subsection{Introduction}

Electrokinetic microfluidics utilize interactions between (bio)particles or fluids and electric fields to achieve electroosmosis, dielectrophoresis, and other forms of electrophoresis. These phenomena have proven to be effective in multiple applications such as electroosmotic flow pumping [44] [143] and (bio)particle separation or concentration [31,32]. Alternating current (AC) electric fields are widely used in those applications due to advantages of relatively stable medium conditions $(\mathrm{pH}$, ionic concentration and ionic strength). Stability is based on the assumption that infinitely small Faradaic currents occur during AC electrokinetic processes.

Reaction kinetics and electrical double layer (EDL) processes play key roles in determining AC Faradaic reaction rates in microfluidics systems. Faradaic reactions are electrochemical reactions at electrode surfaces where ions are transferred into or out of electrolyte solutions thus causing oxidation or reduction [144].

2 This chapter is a reproduction of an article which was published on Biomicrofluidics in Mar 2014.

Biomicrofluidics, 2014. 8(6)

DOI: $10.1063 / 1.4904059$

Reproduced by permission of The Royal Society of Chemistry 
In addition to Faradaic reactions, EDLs establish at the interface between charged electrodes and electrolyte solutions as reviewed previously $[144,145]$. For positively charged electrodes, the EDL includes a compacted layer of strongly absorbed anions adjacent to the electrode called the Stern layer. This layer is composed of both specifically adsorbed ions, governed by electrode material adsorption of the ions, and non-specifically adsorbed counterions, governed by Coulomb forces. Beyond the Stern layer is the diffuse layer, which includes both anions and cations freely transporting in the electrolyte solution via thermal motion and electrostatic interactions. In this model, the inner Helmholtz plane (IHP) is the distance equal to the radius of a non-specifically adsorbed anion from the charged electrode and the outer Helmholtz plane (OHP) is a distance from the electrode equal to the radius of a specifically adsorbed ion plus the solvent molecule diameter.

Grahame's review [53] examined the relationship between electrode processes and EDL, and proved that ion reduction occurs within the EDL instead of the solution interior and the reaction rate-related concentration should be at the outer Helmholtz plane instead of in the bulk solution. Further, the EDL does not reach equilibrium instantaneously. Instead, it is established during a finite electrode charging time, which is given by the product of typical resistance of bulk electrolyte $L / \sigma S[59,146]$ and the typical capacitance of EDL, $\varepsilon S / \lambda_{D}$ :

$$
t_{c}=\left(\frac{\varepsilon}{\sigma}\right)\left(\frac{L}{\lambda_{D}}\right) \quad \text { and } f_{c}=\frac{1}{t_{c}}
$$

where $\varepsilon$ and $\sigma$ are the fluid permittivity and conductivity, $L$ and $S$ are 
the system's characteristic length and area, respectively, and $\lambda_{D}$ is the Debye length, which for the monovalent electrolyte can be expressed as:

$$
\lambda_{D}=\sqrt{\frac{\varepsilon R T}{2 F^{2} C_{0}}}
$$

where, $R$ is gas constant, $T$ is temperature, $F$ is Faradaic constant, and $C_{0}$ is bulk molar concentration.

Thus, the significant period, $\mathrm{T}$, of the externally applied electric field should be regarded as a relative value to $t_{c}$ in Hertz. For example, the applied signal period relative to $t_{c}$ was reported as the determining factor for ACEO pumps [146], As a result, $f_{c}=\frac{1}{t_{c}}$ is the electrode characteristic charging frequency which is equal to the inverse charging time, $t_{c}$.

When the frequency of an applied electric potential is much less than the charging frequency, $f \ll f_{c}$, EDL is well established and the Faradaic reaction progresses with ions from the $\mathrm{EDL}$ at rates close to $\mathrm{DC}$ conditions. On the opposite extreme, when $f \gg f_{c}$, there is not enough time for EDLs to be established and the reaction rate is negligible. This can also be understood from a simplified electrode/electrolyte interface model, in which a capacitor, which represents the EDL, is connected in parallel with a resistor, which represents charge transfer across the interface. At higher frequencies, most of the current to the electrode charges the EDL when the EDL shows low impedance. Thus most of the potential drop occurs within the bulk electrolyte instead of the EDL, which results in negligible Faradaic current before polarity reverses in the AC field [147]. 
Within the bulk solution, the constant, uniform concentration assumption has been questioned. Ramos et al. [121] and Studer et al. [148] found that flow reversal occurred during experiments on $\mathrm{AC}$ electroosmosis (ACEO) pumps. Garcia-Sanchez et al. [121] proposed and experimentally demonstrated in parallel electrode arrays below $f_{c}$ that such flow reversal was induced by an AC Faradaic reaction; this was subsequently verified by $\mathrm{Ng}$ et al. [131] by experimentally detecting $\mathrm{pH}$ in AC electrokinetic systems.

Faradaic reactions have previously been detected by examining solution $\mathrm{pH}$ changes: Sanchez et al. [121] detected significant $\mathrm{pH}$ change at $500 \mathrm{~Hz}$ using $0.1 \mathrm{mM} \mathrm{KCl}$ solution in $10 \mu \mathrm{m}$ and $20 \mu \mathrm{m}$ characteristic length systems, deducing $f_{c} \approx 3 \mathrm{kHz}$. Ng et al. [131] detected $\mathrm{pH}$ change at $100 \mathrm{~Hz}$ using $1 \mathrm{mS} / \mathrm{m} \mathrm{KCl}$ solution in a characteristic length system of $80 \mu \mathrm{m}$, deducing $f_{c} \approx 0.6 \mathrm{kHz}$. These previous experiments were conducted at frequencies well below $f_{c}$, where the EDL is well established and Faradaic reactions are expected and predicted by theory.

However, in microfluidic devices, many applied frequencies are close to or higher than $f_{c}$. For ACEO pumps $[59,143]$, maximum velocities are observed around $f_{c}$. For a majority of (bio)particle DEP characterizations, operational and crossover frequencies are much greater than $f_{c}$, although cellular changes attributed to ion non-uniformities have been quantified at frequencies closer to $f_{c}$ [108]. Thus, to better understand solution behaviors and facilitate intelligent device designs, Faradaic reaction behaviors need to be examined at frequencies around and higher than $f_{c}$. 
Faradaic reactions in parallel electrode arrays generating spatially uniform $\mathrm{pH}$ gradients $[121,131]$ are fairly well explored, while spatially non-parallel electrode systems are unexplored to the authors' best knowledge. Many ACEO pumps and all DEP manipulations require spatially non-uniform electric fields. Thus, 2D non-uniform electric field Faradaic reaction behaviors must be characterized over a range of frequencies and electric field path lengths to discern variations in Faradaic reaction rates. In this work, $4 \mathrm{mM} \mathrm{NaCl}$ solutions $(0.048 \mathrm{~S} / \mathrm{m}$, relative permittivity of 77.98) were explored within a microdevice with the smallest characteristic length of $100 \mu \mathrm{m}$; these conditions yield a maximum characteristic electrode charging frequency of $\sim 3.3 \mathrm{kHz}$.

For electrokinetic microfluidics, water electrolysis is often the most dominant Faradaic reaction [7, 8] as studied in previous works [149-151]. $\mathrm{pH}$ changes are induced by water electrolysis according to the anode and cathode redox reactions:

$$
\begin{aligned}
& \text { Anode: } 2 \mathrm{H}_{2} \mathrm{O} \leftrightarrow \mathrm{O}_{2}(\mathrm{~g})+4 \mathrm{H}^{+}+4 \mathrm{e}^{-} \\
& \text {Cathode: } 2 \mathrm{H}_{2} \mathrm{O}+2 \mathrm{e}^{-} \leftrightarrow \mathrm{H}_{2}(\mathrm{~g})+2 \mathrm{OH}^{-}
\end{aligned}
$$

Thus, to remain consistent with prior Faradaic reaction measurements, we also detect $\mathrm{pH}$ changes in solutions. We first perform qualitative control experiments to detect the impact from electric field shape and solvent material to illustrate that water electrolysis dominates the Faradaic current. Then, we quantitatively detect $\mathrm{pH}$ change in 2D spatially non-uniform 
electric fields created by orthogonally positioned electrode pairs via time analysis. Finally, we detect $\mathrm{pH}$ change at frequencies from $0.9 f_{c}$ to $5 f_{c}$ to examine how Faradaic reaction performs around and above $f_{c}$.

\subsection{Materials and Methods}

\subsubsection{Materials}

Fluorescein sodium salt $(460 / 515 \mathrm{~nm})$, a $\mathrm{pH}$ dependent dye with detection range above $\mathrm{pH}=4$, was used to detect $\mathrm{pH}$ change in real time. Fluorescein sodium salt (Sigma-Aldrich, USA) was prepared to $10^{-5} \mathrm{M}$ in both e-pure water $(\mathrm{pH}=7)$ and methanol $(\mathrm{MeOH}, 99.99 \%$ Sigma-Aldrich, USA).

$4 \mathrm{mM} \mathrm{NaCl}(>99 \%$ pure, Macron Chemicals, USA) aqueous solutions with $\mathrm{pH}$ was adjusted to $7.0 \pm 0.1$ with $1 \mathrm{M} \mathrm{NaOH}$ (Sigma Aldrich, USA) or $1 \mathrm{M} \mathrm{HCl}$ (Sigma Aldrich, USA). $4 \mathrm{mM} \mathrm{NaCl} \mathrm{MeOH}$ solution was similarly prepared.

\subsubsection{Microdevices Design and Fabrication}

Orthogonally positioned $\mathrm{Ti} / \mathrm{Au}(50 / 50 \mathrm{~nm}$ thickness) electrode pairs with $100 \mu \mathrm{m}$ gap created 2D spatially non-uniform electric fields. Standard soft photolithography fabrication procedures were followed [108]. Briefly, photoresist was spun, masked, and exposed to create the necessary electrode features. Next, a Ti/Au layer $\sim 200 \mathrm{~nm}$ thick was sputtering, then remaining photoresist removed via acetone sonication. A PDMS fluidic layer was molded over a silicon wafer prepared by spinning photoresist to $70 \mathrm{uM}$ thick, masking and exposing to UV, 
baking, and then removing unpolymerized photoresist. The PDMS fluidic layer was adhered after air plasma bonding to the glass slide with electrode features. Fig. 20 shows a) an entire device photo and b) 10X magnified view of experimental test chamber. AC signals were generated via an Agilent 33250A waveform generator across the vertical electrode $\left(\mathrm{V}_{\mathrm{pp}} \sin (\omega \mathrm{t})\right)$ and horizontal electrode (grounded).

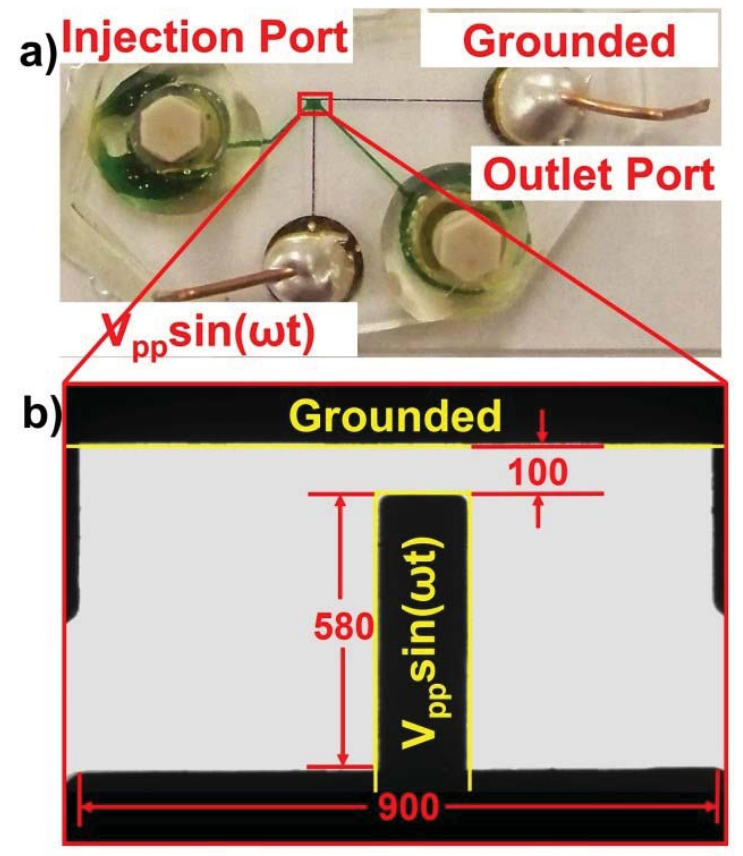

Figure 20: Microdevice designa): Photo of device, with fluid channel filled with green dye, red boxes the working fluidic chamber and $b$ ) Red boxed area under 10x microscope. Units for all numbers are micrometers

\subsubsection{Experiments}

Fluorescein at $0.4 \mu \mathrm{M}$ was calibrated from $\mathrm{pH} 4$ to 8 in $4 \mathrm{mM}$ $\mathrm{NaCl}$ adjusted with $\mathrm{NaOH}$ or $\mathrm{HCl}$. All fluorescent imaging experiments were completed with a Zeiss Axiovert 200m microscope. Videos were recorded with an AxioCam MRm camera and fixed exposure times 
between 250-290 ms. Fluorescein photobleaching was quantified by recording emission light intensity every $1 \mathrm{sec}$ under exposure to excitation light for $120 \mathrm{~s}$.

Samples for $\mathrm{pH}$ change experiments were prepared with 4:96 Fluorescein and aqueous $4 \mathrm{mM} \mathrm{NaCl}$ solution; Control experiments utilized mixture of Fluorescein in $\mathrm{MeOH}$ phase and $4 \mathrm{mM} \mathrm{NaCl}$ also in $\mathrm{MeOH}$ at the ratio of 4:96. Experimental samples were injected via an input port shown in Fig. 20(a) then sealed with One-Piece Fitting (LabSmith, USA) to prevent any net pressure driven flow. The device was mounted on the microscope stage. AC signals ranging in amplitude of 3.5 to $7.5 \mathrm{~V}_{\mathrm{pp}}$ and frequency ranging from 3 to $12 \mathrm{kHz}$ were applied. Microscope videos at 10x magnification were recorded at $1 \mathrm{fps}$ for 120 seconds; no electric fields were applied in the first 5 frames to check system stability. Each experiment was repeated 5 times.

To demonstrate the difference in $\mathrm{pH}$ change behavior between $2 \mathrm{D}$ uniform and non-uniform electric fields, parallel electrode pairs were utilized to create uniform electric fields at $5 \mathrm{kHz}, 5.5 \mathrm{Vpp}$ in both aqueous and $\mathrm{MeOH}$ phases.

\subsubsection{Data Analysis Methods}

\section{Intensity Analysis}

Both 2-D gray scale images and 3-D mesh images were utilized to demonstrate averaged data from 5 repeated experiments at each pixel. For time analysis and control experiments, recorded videos (121 frames 
for 0 to 120s) were exported as individual images, then imported into MATLAB to generate gray scale matrixes ranging from 0 (dark) to 255 (bright). For each experiment, an average pixel value was obtained over 5 repeats and standard deviation was calculated by pixel. MATLAB output was either a 2-D reformed image of 5 superimposed repeats or a 3-D mesh plot with z-axis expressing emission intensity. Also, intensity $/ \mathrm{pH}$ change was determined by subtracting intensity at $120 \mathrm{~s}$ from intensity at $8 \mathrm{~s}$. Time $=8 \mathrm{~s}$ was chosen as a basis due transient higher emission intensity induced by fluorescein molecule enrichment as discussed in section 3.3.

For peak-to-peak potential and frequency dependency analysis, centerline intensity was obtained between two electrodes (50 $\mu \mathrm{m}$ away from each electrode), then averaged intensity was calculated and normalized to range from 0 to 255 . Similarly, each pixel was averaged from 5 repeats with standard deviation.

\section{Converting Intensity to $\mathrm{pH}$}

Obtained intensities were corrected with a derived photobleaching equation to exclude the intensity drop induced by FITC degradation with light. Then, $\mathrm{pH}$ values at each time and position were calculated from the corrected intensity via Fig. 21(c) calibration. 

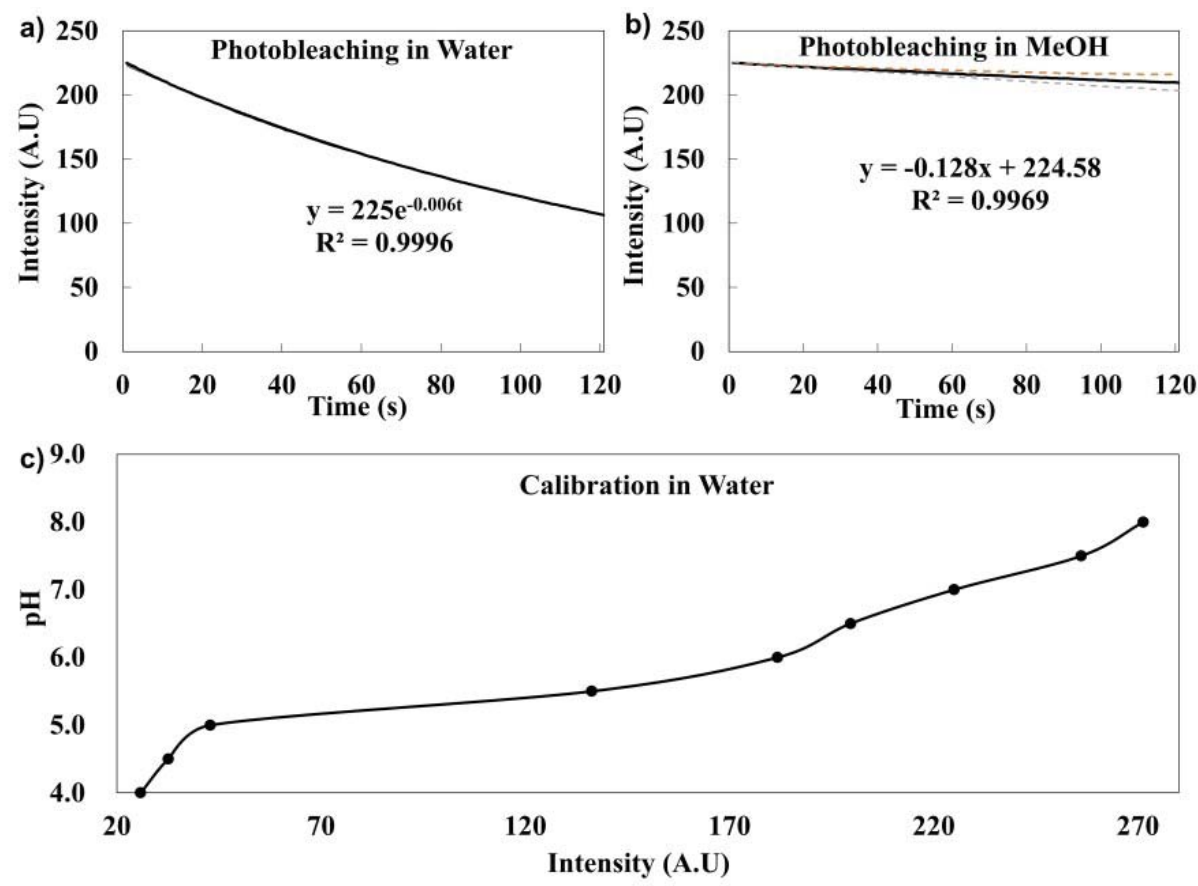

Figure 21: Dye properties study: a): Photobleaching property in water, b) Photobleaching property in methanol and c): $\mathrm{pH}$ calibrated to dye emission intensity

\subsection{Results and Discussion}

To capture the most dominant electrochemical reaction behavior and to be consistent with published data, $\mathrm{pH}$ was detected to track Faradaic reactions. $\mathrm{pH}$ sensitive fluorescein salts were utilized to quantify photobleaching effects and to obtain fluorescein emission intensity versus $\mathrm{pH}$ calibration curve. Comparison experiments were performed to exclude potential emission intensity side mechanisms including photobleaching, temperature changes, induced flows or other phenomena induced by non-uniform electric fields. Intensity measurements were converted into $\mathrm{pH}$ correcting for photobleaching, then position distributions were mapped in the device. Finally, signal magnitude and frequency dependencies were 
quantified.

\subsubsection{Fluorescein Calibrations}

The fluorescein $\left[\mathrm{H}^{+}\right]$ion indicator controls were completed to account for photobleaching, background, and intensity calibrations. First, photobleaching properties were examined by detecting emission intensity without electric fields in both aqueous solution (Fig. 21(a)) and $\mathrm{MeOH}$ solution (Fig. 21(b)). Photobleaching in each solution was quantified from 5 repeats; Fig. 21 results illustrate the average value. The standard error was less than 5\% in water while the upper and lower limits in $\mathrm{MeOH}$ were plotted. Fluorescein emission intensity decreased from 225 to $\sim 120$ (arbitrary units) in aqueous solution over $120 \mathrm{~s}$ with no field, while in $\mathrm{MeOH}$, the decrease was only from 225 to 210 . Photobleaching behavior was fit into an exponential or linear function with $\mathrm{R}^{2}>0.995$ for water and $\mathrm{MeOH}$, respectively. The photobleaching equations shown in Fig. 21 were utilized to remove the photobleaching effect from all subsequent intensity measurements.

Second, manually pipetted and meter verified $\mathrm{pH}$ solutions were calibrated against emission intensity as shown in Fig. 21(c). Fluorescein emission intensity reduced to zero at $\mathrm{pH}<4$. For $\mathrm{pH}>4$, emission intensity increased with $\mathrm{pH}$. In our experiments, Faradaic currents primarily yielded emission intensity decreases, which correspond to $\mathrm{pH}$ decreases. Intensity data was first scaled from 0 to 255 , then the calibration used to obtain the $\mathrm{pH}$ value. 


\subsubsection{Comparison experiments}

Comparison experiments were conducted to elucidate other potential mechanisms impacting local $\left[\mathrm{H}^{+}\right]$and thus $\mathrm{pH}$ measurement accuracy. In contrast to uniform electric fields, 2D spatially non-uniform electric fields can potentially induce possible side effect depending on experimental parameters such as solution concentration, conductivity, applied potential and frequency. Under the experimental conditions in this work, AC electroosmotic (ACEO) flow is the most probable induced phenomenon due to the low $\mathrm{kHz}$ applied frequencies. ACEO is caused by the applied electric fields exerting force on hydrated ions in the induced EDL thus generating flow movement. Such flow can change emission intensity via mass transfer induced changes in fluorescein concentration. Thus, to accurately quantify $\mathrm{pH}$ change in the system, ACEO effects were examined.

$\mathrm{MeOH}$ can be electrolyzed only in the presence of water [152], so this pure solvent was used for comparison experiments. A mixture of 4 $\mathrm{mM} \mathrm{NaCl}$ in $\mathrm{MeOH}$ and $10^{-5} \mathrm{M}$ fluorescein in $\mathrm{MeOH}$ was employed. Negligible Faradaic reaction products were detected in the $\mathrm{MeOH}$ system as illustrated in Fig. 22. This enabled determination of any phenomena other than Faradaic reactions inducing intensity changes in the non-uniform electric fields. 

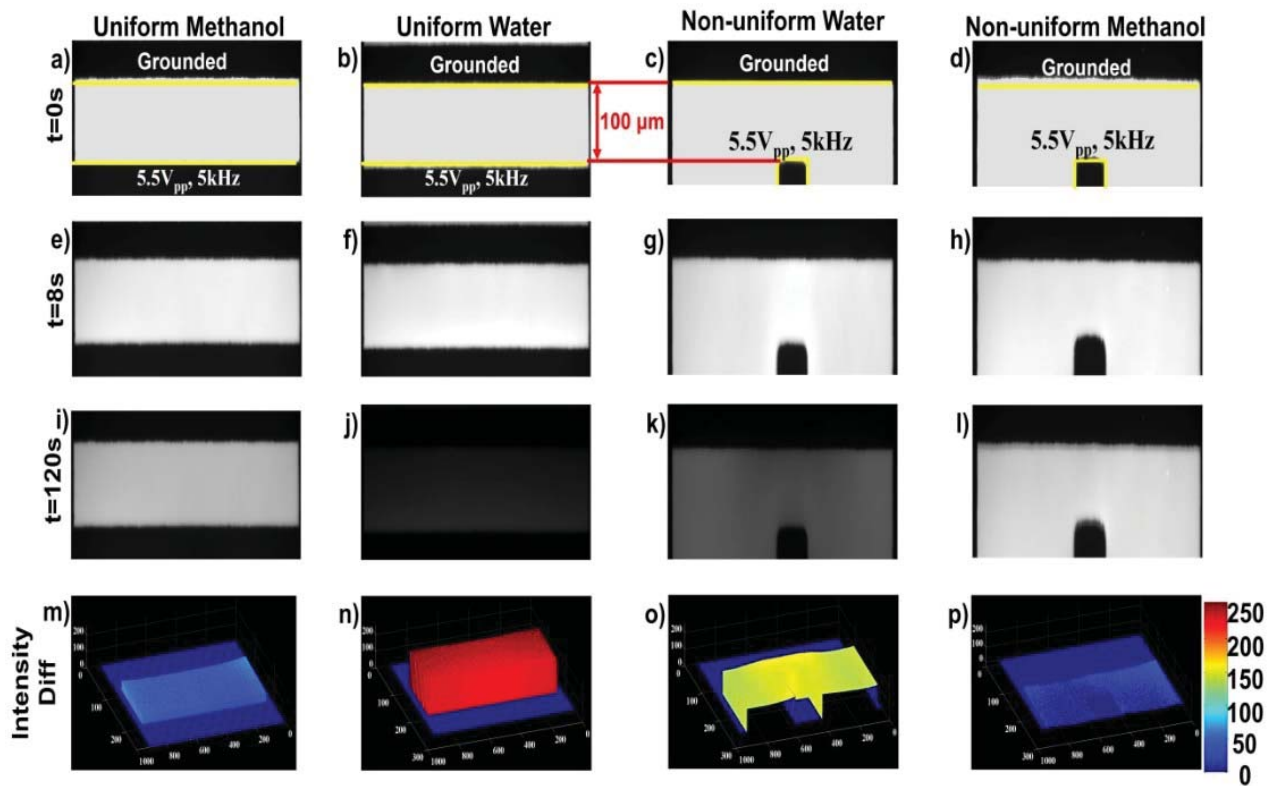

Figure 22: Comparison experiments under $5 \mathrm{kHz}, 5.5 \mathrm{Vpp}$ :First column are $\mathrm{t}=0,8,120$ s and intensity difference obtained by MATLAB image analysis of experiments under uniform electric field in methanol solvent; Second column are same time and image output of experiments under uniform electric field in

water solvent. Third column are same information of experiments under non-uniform electric field in water solvent and fourth column are same information under non-uniform electric field in methanol solvent. It can be observed that intensity change can be observed in $\mathrm{n}$ ) and o) while no change observed in $\mathrm{m}$ ) and $\mathrm{p}$ )

To examine Faradaic reactions above the charging frequency $\left(f_{c}>3.3\right.$ $\mathrm{kHz}$ ) in both 2D spatially uniform and non-uniform electric fields, both $\mathrm{MeOH}$ and aqueous solutions were observed under $5.5 \mathrm{~V}_{\mathrm{pp}}$ and $5 \mathrm{kHz}$ sinusoidal AC signals (relative frequency $=1.5 f_{c}$ ). Fig. 22 shows 5 repeated control experiment results already corrected for photobleaching. For all combinations of solvents and electric field shape, results at $\mathrm{t}=0,8$, and $120 \mathrm{~s}$ are shown in Fig. 22 (a-d), (e-h), and (i-1), respectively. Fig. 22 (m-p) shows intensity differences between 
$\mathrm{t}=8 \mathrm{~s}$ and $\mathrm{t}=120 \mathrm{~s}$. Time $=8 \mathrm{~s}$ was chosen as a basis due to fluorescein molecule enrichment as will be discussed in section 3.3.

For $\mathrm{MeOH}$ in uniform electric fields generated via parallel electrode pairs (Fig. 22(m)) and $\mathrm{MeOH}$ in non-uniform electric fields generated via perpendicular electrode pairs, no substantial intensity changes were observed. For the aqueous uniform electric field case (Fig. 22(n)), intensity changes up to 200 arbitrary intensity units (AU) were observed. For the aqueous non-uniform electric field case (Fig. 22(o)), an intensity change of $\sim 150 \mathrm{AU}$ was observed. These intensity changes were consistent with $\mathrm{pH}$ shifts generated by Faradaic reactions. The smaller intensity change in the nonuniform case is likely the result of the orthogonal effective electrode surface area being smaller than the parallel electrode surface area. Since the Faradaic reactions are electrode surface area-driven, this result is consistent with Faradaic $\mathrm{pH}$ products. For the $\mathrm{MeOH}$ non-uniform electric field case (Fig. 22(p)), in which ACEO flow was possible, the emission intensity change was negligible. Fig. 22(m) shows that in the $\mathrm{MeOH}$ phase, negligible intensity change - beyond the $8 \mathrm{~s}$ fluorescein molecule enrichment was observed when compared to water phase results in Fig. 22(n). Such observations are consistent with negligible Faradaic electrolysis reactions in water-free, yet ion rich $\mathrm{MeOH}$. Further, other non-uniform electric field side effects in ionic solutions such as ACEO, thermal convection flows or other solution property change are not evident.

These comparison experiments reveal that the observed intensity 
changes in Fig. 22(o) can be confidently correlated with $\mathrm{pH}$ changes generated by Faradaic reactions and not fluid transport at frequencies greater than the electrode charging frequency in non-uniform electric fields. This, in combination with prior publications [[59]] increases confidence in the fluorescently measured $\mathrm{pH}$ change accuracy and conclusion that Faradaic reactions drive the $\mathrm{pH}$.

\subsubsection{Time analysis of intensity change}

Since the intensity change can be directly correlated with Faradaic reactions, further analysis was conducted on time and spatial dependencies within the electric fields. Fig. 23 shows time and spatial results at $5.5 \mathrm{~V}_{\mathrm{pp}}$ and $5 \mathrm{kHz}$ after photobleaching corrections revealing Faradaic reaction-induced intensity changes. Results are organized into columns correspond to experiment time: column 1 is for $\mathrm{t}=0 \mathrm{~s}(\mathrm{a}, \mathrm{e}$, i), column 2 is for $t=8 s(b, f, j)$, column 3 is for $t=60 s(c, g, k)$, and the last column is $\mathrm{t}=120 \mathrm{~s}(\mathrm{~d}, \mathrm{~h}, 1)$. The respective rows from top to bottom are: first row shows 2-D gray scale image averaged pixel by pixel from 5 experimental repeats (a-d), the second row is a 3-D mesh image where the $\mathrm{z}$ height corresponds to the intensity magnitude (e-h), and the third row is a 3-D mesh of the pixel by pixel standard deviation calculated from the 5 experiments (i-1). 

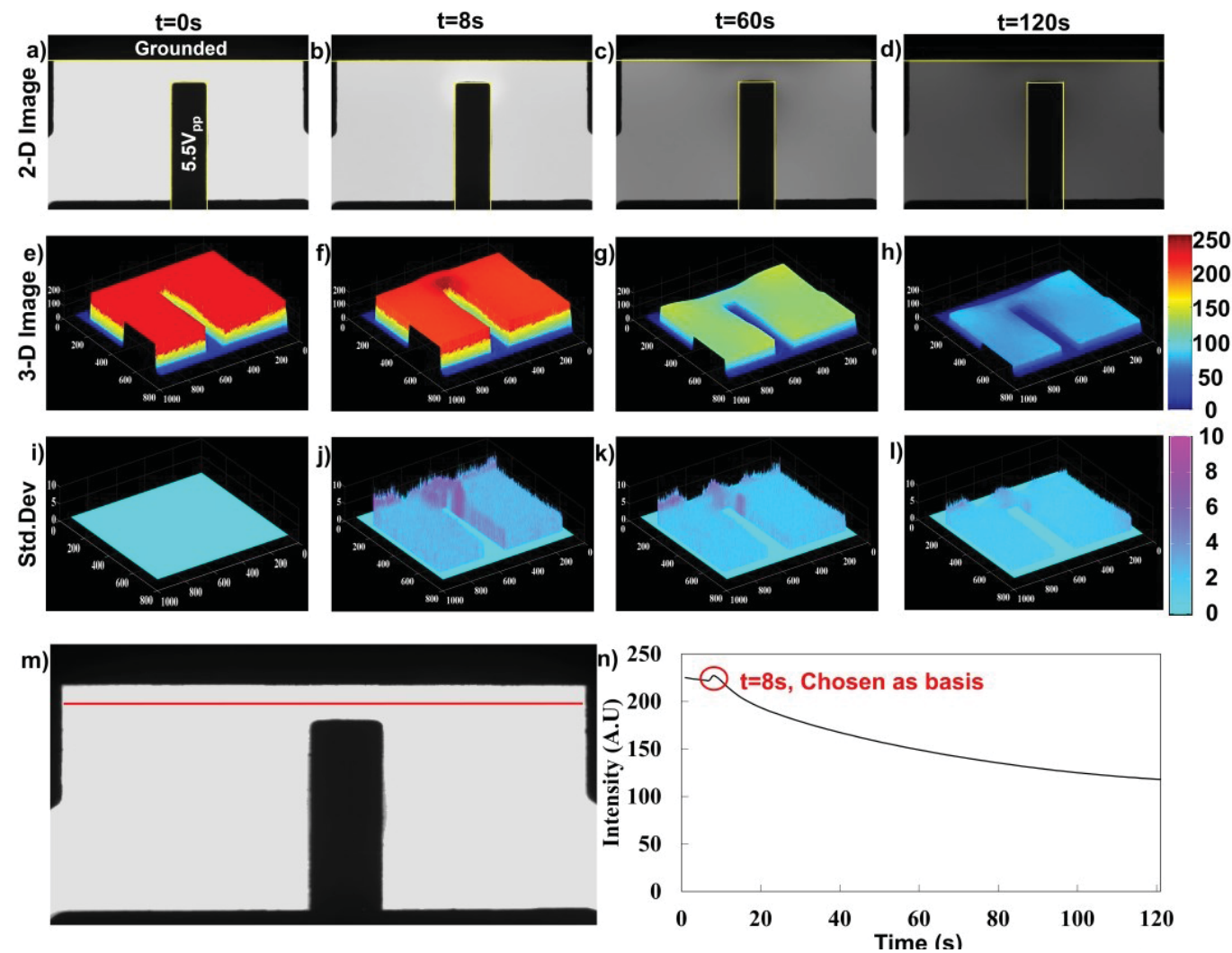

Figure 23: Intensity time analysis: Gray-scale 2-D dye intensity plot obtained from MATLAB image analysis at $\mathrm{t}=0,8,80$ and $120 \mathrm{~s}$ under $5.5 \mathrm{~V}_{\mathrm{pp}} 5 \mathrm{kHz}$; e)-h): 3-D MATLAB plot at same time point and i)-1): Calculated standard deviation of 5 repeats shown in the first two rows. Red line in figure $m$ ) shows the position of sampled line intensity. Figure n) is showing the time dependency of the average of sampled line intensity.

For the first column at $\mathrm{t}=0$, images illustrate the spatially uniform fluorescein emission intensity when no electric field was applied; for comparisons between runs, this initial intensity was always normalized to $225 \mathrm{AU}$. These baseline images represent the microdevice $\mathrm{pH}$ state without influence from Faradaic reactions or electric field effects (other mechanisms were negligible in section 3.2).

To quantify the time dependence of $\left[\mathrm{H}^{+}\right]$concentrations by area, 
Fig. 23 ( $m$ and $n$ ) identify and compare spatial subareas including the left and right peripheral areas, central area, cross-sectional area, and a single line intensity profile within the nonuniform field case. Each subarea or line was tracked frame-by-frame in each separate experiment as shown in Fig. 23(n), and then compiled into trend data for time dependence. Left and right area intensities were steady to $5 \mathrm{~s}$, then once the electric field was applied, monotonically decrease from 225 to $\sim 180$ by $120 \mathrm{~s}$. Central and cross-sectional areas and line profile intensities also started from 225 and remained steady to $5 \mathrm{~s}$. Upon electric field application, intensities reached a maximum at $\mathrm{t}=8 \mathrm{~s}$. Since trends were similar, the simpler line intensity was selected to sufficiently capture aggregate trends for all subsequent time, potential and frequency dependencies. Since the measured intensity of the fluorescein directly correlates with $\mathrm{pH}$ as illustrated in Fig. 21(c), this tool was used to track $\mathrm{pH}$ changes beyond the electrode charging frequency.

The field remained off until $\mathrm{t}=5 \mathrm{~s}$, but within 3 seconds of $5.5 \mathrm{~V}_{\mathrm{pp}}$ and $5 \mathrm{kHz}$ electric field application, a higher emission intensity was observed in the central area while intensity was unchanged elsewhere. This spatial variation was attributed to fluorescein molecular movement to this high electric field density region. Reasoning for this is threefold: 1) As shown in Fig. 22, experiments completed in $\mathrm{MeOH}$ also demonstrated a small intensity increase after $3 \mathrm{~s}$ in the field, 2) In this $\mathrm{MeOH}$ system, no further intensity changes were observed after $8 \mathrm{~s}$, and 3) In uniform electric field experiments, no spatial changes were 
discernable in either $\mathrm{MeOH}$ or water. These three clues suggest this $3 \mathrm{~s}$ increase is directly related to the electric field non-uniformity and not the result of any electrochemical reactions, since such reactions would progress with time. Although this non-Faradaic phenomenon is not a major focus of this work, it still impacts the intensity measurements necessary to quantify progression of the Faradaic reactions. Thus, the higher intensities at $8 \mathrm{~s}$ were used as a baseline to more accurately map the Faradaic reaction effects in the system and decouple the fluorescein concentration effects from $\mathrm{pH}$ change effects as shown in Fig. 23(n).

Progressing up to $\mathrm{t}=60 \mathrm{~s}$, significant intensity decrease was observed throughout the entire observed device chamber; the most significant intensity decreases were observed in the central area. This intensity decrease was attributed to increasing $\left[\mathrm{H}^{+}\right]$per the calibration in Fig. 21 (c). The $\left[\mathrm{H}^{+}\right]$products indicate Faradaic reactions occurring at $5.5 \mathrm{~V}_{\mathrm{pp}}$ and $5 \mathrm{kHz}$, beyond the electrode charging frequency. Also, spatially non-uniform intensities indicate that the reaction rate varies spatially; reaction rate is higher in the central area and lower in the peripheral areas as illustrated in Fig. 22(g). This spatial variance of reaction rate is induced by the electric field non-uniformity and more specifically, by the non-uniformity of the characteristic length of the electric field lines, which governs the path for ion motion between electrode surfaces. The ion path length in the central area is much shorter enabling the charges to reach the electrode surface much faster in each $\mathrm{AC}$ cycle giving sufficient time for the molecules to participate in the Faradaic reaction. In the peripheral areas, charges are transported 
along longer electric field path lines and thus reach the electrode surface later in the $\mathrm{AC}$ cycle resulting in attenuated Faradaic reactions. Since 2D nonuniform electric fields have varying characteristic lengths, and as reinforced by equation 1 and 2, longer characteristic lengths result in higher electrode charging frequencies. For any given applied signal frequency, the relative frequency $\left(f / f_{c}\right)$ that the solution experiences varies spatially due to this electric field non-uniformity, thus resulting in a spatially different Faradaic reaction rate.

Observations to $120 \mathrm{~s}$ ( $4^{\text {th }}$ column in Fig. 23) reveal further decreases in the emission intensity, which supports that continual Faradaic reaction products accumulate in the solution. Comparisons between the central area of shortest electric field path lines (Fig $23 \mathrm{~m}$ ) and peripheral areas with longer electric field path lines reveal spatially non-uniform $\left[\mathrm{H}^{+}\right]$as a result of the spatially varied reaction rate.

\subsubsection{2-D Mapping of $\mathrm{pH}$ Change}

Using the $\mathrm{pH}$ value obtained from the Fig. 21 (c) calibration curve, $\mathrm{pH}$ was mapped as a function of time and position. Also, to increase accuracy, $\mathrm{pH}$ change $\left(\Delta \mathrm{pH}=\mathrm{pH}_{\mathrm{t}=8 \mathrm{~s}}-\mathrm{pH}_{\mathrm{t}=\mathrm{ts}}\right)$ was demonstrated instead of an absolute $\mathrm{pH}$ value to further exclude molecular accumulation effects prior to $\mathrm{t}=8 \mathrm{~s}$. Absolute $\mathrm{pH}$ was then calculated from the difference from the starting neutral $\mathrm{pH}$ via $\mathrm{pH}=7-\Delta \mathrm{pH}$. 

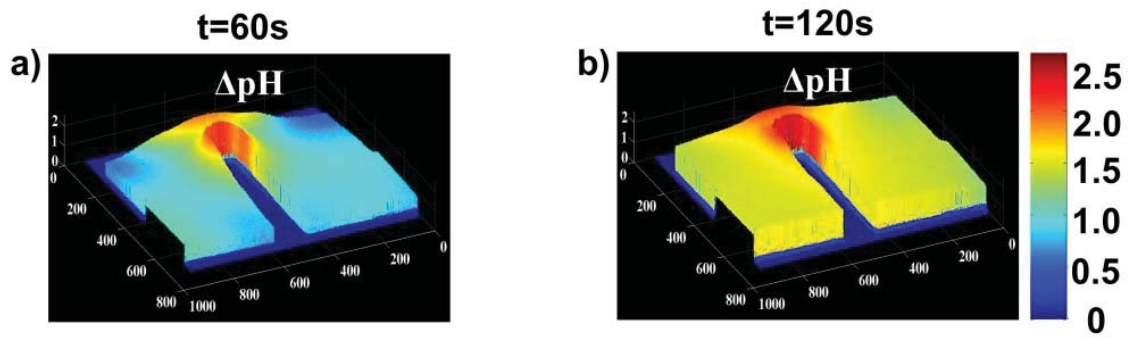

c)

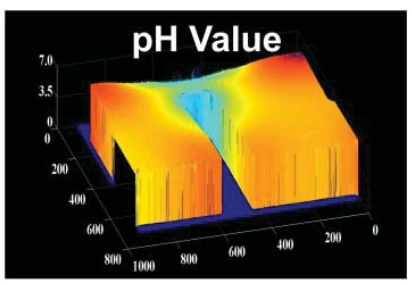

d)

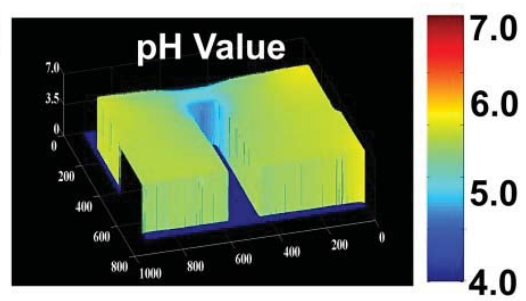

e)
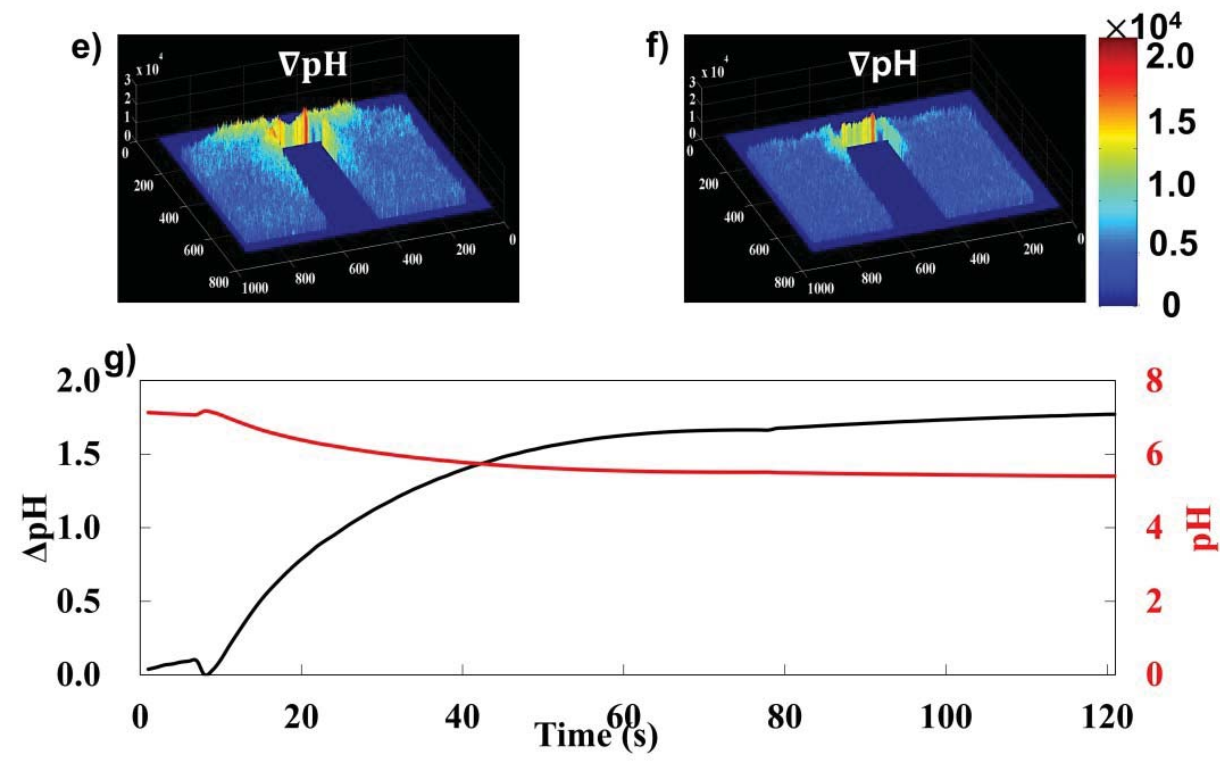

Figure 24: $\mathrm{pH}$ change time analysis:a) and b): $\mathrm{pH}$ change, c) and d): absolute $\mathrm{pH}$ value, e) and d): $\mathrm{pH}$ gradient at time $=60 \mathrm{~s}$ and $120 \mathrm{~s}$, respectively. Figure $\mathrm{g}$ ) shows time dependence of $\mathrm{pH}$ change (black line) and absolute $\mathrm{pH}$ value (red line)

Fig. 24(a) and 5(b) illustrate $\mathrm{pH}$ differences from $5.5 \mathrm{~V}_{\mathrm{pp}}$ and 5 $\mathrm{kHz}$ between $\mathrm{t}=8$ and $60 \mathrm{~s}$ as well as $\mathrm{t}=8$ and $121 \mathrm{~s}$, respectively. Fig. 24(c) and 5(d) show $\mathrm{pH}$ values obtained from a uniform neutral $\mathrm{pH}$ everywhere at $\mathrm{t}=0 \mathrm{~s}$. Fig. 24(e) and 5(f) show the geometric $\mathrm{pH}$ 
gradient $(\nabla p H)$. Fig. $24(\mathrm{~g})$ shows both $\mathrm{pH}$ change (black solid line) and absolute $\mathrm{pH}$ (red dashed line) for the average line intensity (labeled in Fig. $23 \mathrm{~m}$ ) with respect to time. Together, Fig. 24 plots illustrate that spatial variations of $\mathrm{pH}$ change, $\mathrm{pH}$ value, and thus the $\mathrm{pH}$ gradient are more substantial than Fig. 23 conveys. At $\mathrm{t}=60 \mathrm{~s}$, the $\mathrm{pH}$ change was $>1.5 \log (\mathrm{pH} \leq 5.5)$ in the central area, while $\mathrm{pH}$ changes were $\sim 1$ in the peripheral areas. By $\mathrm{t}=120 \mathrm{~s}, \mathrm{pH}$ change increased to $>2(\mathrm{pH}<5)$ in the central area and $\sim 1.5$ in the periphery.

Fig. 24(e) and 4.5(f) show the geometric $\mathrm{pH}$ gradient or spatial variance of $\mathrm{pH}$ change. At $\mathrm{t}=60 \mathrm{~s}$, the central area $\nabla(p H)$ value was twice that in the surrounding area illustrating widespread spatial variations in $\mathrm{pH}$. By $\mathrm{t}=120 \mathrm{~s}$, however, large $\mathrm{pH}$ gradients are only observed in the central area, indicating that solution $\mathrm{pH}$ became more uniform and reached equilibrium in peripheral regions. To better elucidate the time dependence, Fig. 24(g) shows the time dependent averaged $\mathrm{pH}$ change and real $\mathrm{pH}$ value in bulk solution $50 \mu \mathrm{m}$ from both electrodes. Bulk pH changes rapidly in the first $60 \mathrm{~s}$ suggesting Faradaic reaction rates are fastest initially, but slow as products accumulate. For $5.5 \mathrm{~V}_{\mathrm{pp}}$ and $500 \mathrm{kHz}$, the $\left[\mathrm{H}^{+}\right]$change was 1.5 orders of magnitude by t=60 s. From 60 to $120 \mathrm{~s}$, the Faradaic reaction rate gradually decreased such that a $\mathrm{pH}$ change of only $\sim 0.25$ took place between 60 and $120 \mathrm{~s}$.

In summary, due to system geometry, the characteristic lengths of ion paths along electric fields lines vary by position. In the central 
region, $100 \mu \mathrm{m}$ was the shortest path yielding the shortest electrode charging time in the system, corresponding to $f_{c}=3.3 \mathrm{kHz}$. Whereas in the peripheral regions, path lengths could reach $300 \mu \mathrm{m}$, corresponding to $f_{c}$ on the order of $1.1 \mathrm{kHz}$. Further, experimental results showed $\mathrm{pH}$ changes of more than 2 units existed at $5 \mathrm{kHz}$. A pH change of 1.5 units within the first $60 \mathrm{~s}$ gives a change in $\left[\mathrm{H}^{+}\right]$of $\sim 3 \times 10^{-6} M$ in the first $60 \mathrm{~s}$ and $\sim 5.5 \times 10^{-6} M$ by $120 \mathrm{~s}$. This infers an average Faradaic reaction rate of $5.1 \times 10^{-8} \mathrm{M} / \mathrm{s}$ for the first $60 \mathrm{~s}$ and $4.6 \times$ $10^{-8} \mathrm{M} / \mathrm{s}$ for the entire $120 \mathrm{~s}$ experiment.

\subsubsection{Potential and Frequency Dependency}

Peak-to-peak $\left(\mathrm{V}_{\mathrm{pp}}\right)$ potential and frequency dependencies of $\mathrm{pH}$ changes were also quantified. $\mathrm{V}_{\mathrm{pp}}$ ranged from $3.5 \mathrm{~V}_{\mathrm{pp}}$ to $7.5 \mathrm{~V}_{\mathrm{pp}}$ at a fixed frequency of $5 \mathrm{kHz}$ since negligible $\mathrm{pH}$ change was observed below $3.5 \mathrm{~V}$ and above $7.5 \mathrm{~V}$ severe electrode damage occurred. Next, frequencies from 3 to $12 \mathrm{kHz}$ (relative frequency $=0.9$ to 4 ) at a fixed applied potential of $5.5 \mathrm{~V}_{\mathrm{pp}}$ were tested to examine Faradaic reaction behavior around and above the electrode charging frequency. The $\mathrm{pH}$ change between $\mathrm{t}=8 \mathrm{~s}$ and $120 \mathrm{~s}$ was tracked with line intensity data (Fig. 23(m)) as a function of position as shown in Fig. 25. Each condition in Fig. 25(a) and 4.6(b) contains 5 repeats with error bars representing standard deviations. 

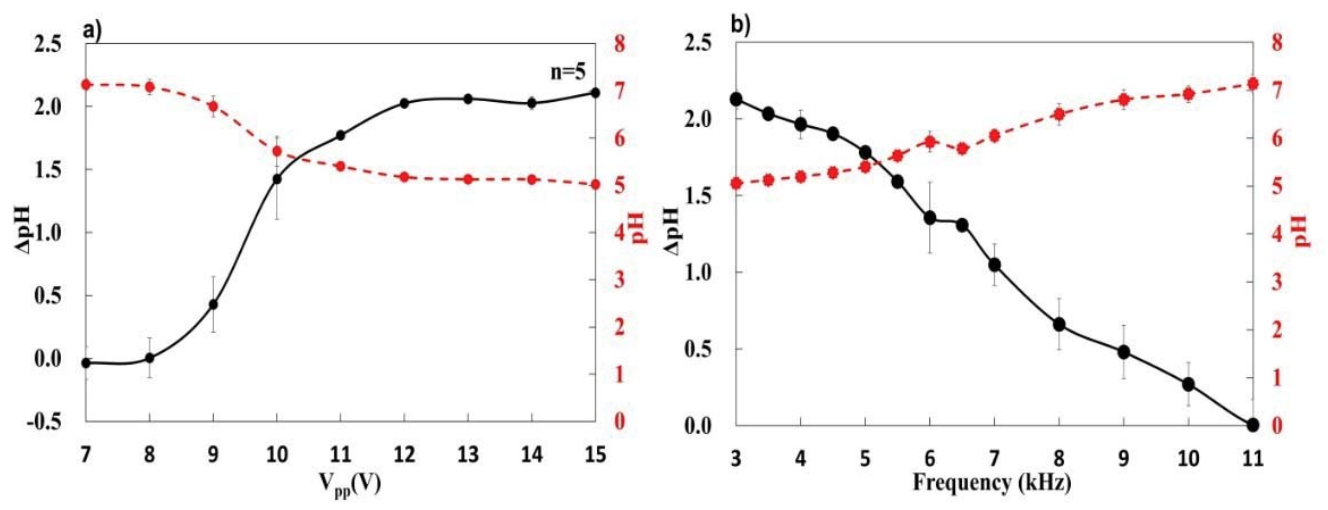

Figure 25: a): potential dependency of $\mathrm{pH}$ change (black) and absolute $\mathrm{pH}$ value (red) at $5 \mathrm{kHz}$ and b): frequency dependency of $\mathrm{pH}$ change (black) and absolute $\mathrm{pH}$ value (red) at $5.5 \mathrm{Vpp}$. a): potential dependency of $\mathrm{pH}$ change (black) and absolute $\mathrm{pH}$ value (red) at $5 \mathrm{kHz}$ and b): frequency dependency of $\mathrm{pH}$ change (black) and absolute $\mathrm{pH}$ value (red) at $5.5 \mathrm{Vpp}$. This shows $\mathrm{pH}$ change increase with peak-to-peak potential and decrease with frequency, which implies the Faradaic reaction increase with peak-to-peak potential and decrease with frequency.

$\mathrm{V}_{\mathrm{pp}}$ dependency results in Fig. 25(a) showed that $\mathrm{pH}$ changes, and thus Faradaic reaction rate, increased with peak-to-peak potential. The reaction rate was negligible at $3.5 \mathrm{~V}_{\mathrm{pp}}$ and started to increase at $4 \mathrm{~V}_{\mathrm{pp}}$. From 4 to $6 \mathrm{~V}_{\mathrm{pp}}$, the $\mathrm{pH}$ change increased rapidly from 0.1 to 2 . At 6 $\mathrm{V}_{\mathrm{pp}}$, reaction rate slowed to negligible above $7.5 \mathrm{~V}_{\mathrm{pp}}$.

Frequency dependence results in Fig. 25(b) showed that reaction rate decreased linearly with frequency. Faradaic reaction products were considerable at frequencies above the presumed $3.3 \mathrm{kHz}$ electrode charging frequency. In fact, $\mathrm{pH}$ changes were observed up to $\sim 11 \mathrm{kHz}$ at which products could no longer be fluorescently detected. 


\subsection{Discussion}

Results from Fig. 24(a) and 4.5(b) indicate that Faradaic reactions are more pronounced in the central area between the electrodes. This is attributed to differences in the ion transfer properties along the non-uniformed ion paths at different lengths represented by the differences in relative frequency $\left(f / f_{c}\right)$ governed by the electrode charging frequency, which is inversely proportional to ion path lengths (Eqn. 1). The shortest ion paths exist where the electrodes are closest, which elicit the lowest charging time and the highest electrode charging frequency. Thus, for a fixed applied frequency across spatially non-uniform electrodes, the relative frequency is lowest in regions with the smallest electrode spacing and higher elsewhere. The applied frequency dependency in Fig. 25(b) shows that lower frequencies yield greater $\mathrm{pH}$ changes. Extending this logic to the relative frequency, which is spatially dependent, it follows that the lower relative frequency in the central area would yield greater Faradaic reactions. Another consequence of this spatial variation is that the farther from the central area, the longer the distance ions need to travel between the two electrodes. This causes a greater charging term, a lower electrode charging frequency, and a higher relative frequency for the same applied frequency.

Increases in relative frequency cause lower reaction rates, evidenced in this work by formation of $\mathrm{pH}$ gradients. Fig. 24(e) and 5(f) illustrate the $\mathrm{pH}$ gradient becomes less pronounced at large time, because $\left[\mathrm{H}^{+}\right]$diffuses throughout (Fig. 24(g)). However, pH gradient 
formation at short time scales provides interesting insights into the Faradaic reactions. It can be inferred from Fig. 24(g) that the Faradaic reaction producing $\mathrm{H}^{+}$becomes equilibrium limited, thus slowing the production of $\mathrm{H}^{+}$. Concurrently, $\mathrm{H}^{+}$diffusion down the $\mathrm{pH}$ gradient occurs on an unchanging time scale $\left(t \approx \frac{x^{2}}{2 D}, \mathrm{D}\right.$ is diffusion coefficient, $\mathrm{x}$ is diffusion length). The $\mathrm{pH}$ gradient decrease can be understood via a relative ion rate ratio $\left(R_{r}\right)$ between the driving reaction rate $\left(R_{r x n}\right)$ and resulting diffusion rate $\left(R_{d}\right)$ as $R_{r}=R_{r x n} / R_{d}$. At $t<60 s, R_{r \times n}>>R_{d}$ in both the central and surrounding area, which leads to higher $R_{r}$ and thus a pronounced $\mathrm{pH}$ gradient. In the peripheral areas, $\mathrm{R}_{\mathrm{rxn}}$ and $\mathrm{R}_{\mathrm{d}}$ are similar orders of magnitude such that $\mathrm{pH}$ gradients are not apparent. From $\mathrm{t}=60$ to 120 s, Fig. 24(f) suggests $R_{r x n}$ decreases. In central areas with minimum characteristic length, $\mathrm{R}_{\mathrm{rxn}}$ remains high, but in peripheral areas, $R_{r x n}$ is reduced. However, $R_{d}$ is a phenomenon responding to the $\mathrm{pH}$ (concentration) gradient such that when the $\mathrm{pH}$ gradient is large, the effective $\mathrm{R}_{\mathrm{d}}$ is also high. As time progresses, the central $\mathrm{pH}$ is relatively constant, but the accumulation of $\mathrm{H}^{+}$in the periphery increases, thus decreasing the $\mathrm{pH}$ gradient and effectively reducing $\mathrm{R}_{\mathrm{d}}$. Thus, $\mathrm{R}_{\mathrm{r}}$ remains high in the central area, while $\mathrm{R}_{\mathrm{r}}$ in the surrounding areas reduces. This combination results in a shrinkage of the high $\mathrm{pH}$ gradient footprint and a $\mathrm{pH}$ uniformity in the peripheral area.

Fig. 25(a) showed how the peak-to-peak potential, at a fixed frequency of $5 \mathrm{kHz}$, impacted the Faradaic reaction rate and thus the resulting $\mathrm{pH}$ change. In this specific electrode configuration, the Faradaic reaction was initiated at $4 \mathrm{~V}_{\mathrm{pp}}(2 \mathrm{~V}$ for each half period); this 
is the 'AC onset potential' for $5 \mathrm{kHz}$. This $\mathrm{AC}$ onset potential is slightly higher than the reported $1.23 \mathrm{~V}$ theoretical standard electrode potential [18] calculated based on reaction thermodynamics and consistent with a previously reported $2 \mathrm{~V}$ experimentally obtained onset potential for a similar gold electrode configuration under DC potential yielding water electrolysis [132]. With increasing overpotential (applied potential standard electrode potential), the Faradaic reaction rate increased significantly, evidenced by the $\mathrm{pH}$ change increasing from 0 to 2.5 . Beyond $6 \mathrm{~V}_{\mathrm{pp}}$, the Faradaic reaction became mass transfer limited such that $\mathrm{pH}$ did not further increase with increasing overpotential.

Fig. 25(b) showed the Faradaic reaction frequency dependence at a fixed applied potential of $5.5 \mathrm{~V}_{\mathrm{pp}}$. Frequencies examined were at 3 $\mathrm{kHz}$ and above the electrode charging frequency. The Faradaic reaction was highly pronounced at $3 \mathrm{kHz}$ and steadily declined to negligible at $11 \mathrm{kHz}$. This trend illustrated that the Faradaic reaction persisted above the charging frequency of $3.3 \mathrm{kHz}$. This can be explained through an electrode-electrolyte interface model with a parallel capacitor and resistor [153]. The capacitor represents the electric double layer (EDL) while the resistor represents electron transfer across the electrode-electrolyte interface, which generates the Faradaic reaction. Previous work was conducted [63, 154-157] at frequencies below the electrode charging frequency, whereby the capacitor remains fully charged in each half period. The fully charged capacitor functions as a high impedance element preventing current passage, while the resistor facilitates electron transfer for the Faradaic 
reaction to progress. At frequencies around $f_{c}$, which this work explored, the capacitor was not fully charged and the EDL was not fully established (electrode not fully polarized/screened). Thus, a similar impedance across the parallel resistor and capacitor results in current passing through both pathways yielding Faradaic reaction products, albeit at an attenuated rate compared to reactions below $f_{c}$. With increases in frequency, the EDL capacitor charges less, favoring current passage through the capacitor over the parallel resistor. The increasing capacitor current thus increasingly bypasses the resistor effectively short circuiting the electrode-electrolyte interface to thus decrease and eliminate the Faradaic reaction rate.

\subsection{Conclusions}

This work spatially and temporally examined Faradaic reactions in spatially non-uniform $\mathrm{AC}$ electric fields via spatial $\mathrm{pH}$ detection at frequencies higher than the theoretical electrode charging frequency.

Faradaic reaction products were observed across the entire microdevice $6.3 \times 10^{5} \mu \mathrm{m}^{2}$ chamber over the course of the $120 \mathrm{~s}$ experiment. The applied frequencies were higher than the theoretically predicted electrode charging frequency meaning that the electric double layer was not well charged. Despite this, $\mathrm{pH}$ changes up to 2.5 orders of magnitude were observed proving the existence of Faradaic reactions above the electrode charging frequency.

Further, spatial variances in the Faradaic reaction rate were observed 
and attributed to different characteristic ion path lengths resulting from the spatial non-uniformity of the electric fields. These spatially varying Faradaic reactions were tracked via observation of $\mathrm{pH}$ gradients reaching magnitudes of $2 \times 10^{4} \mathrm{unit} / \mu \mathrm{m}$.

Lastly, Faradaic reaction rate increases were observed with increasing potential and Faradaic reaction rate decreases were observed with increasing frequency. The reaction rate increased with potential due to increasing electron transport rate from electrode to electrolyte. Reaction rate decreased with frequency due to insufficient time to fully charge the electric double layer in accordance with the $\mathrm{RC}$ electrode-electrolyte behavior model.

This work demonstrates that Faradaic reactions influence the local $\mathrm{pH}$ above the electrode charging frequency. Further, these $\mathrm{pH}$ changes vary with time and position. The implications are that $\mathrm{pH}$ gradients may induce anomalous (bio)particle dielectrophoretic responses and hydrodynamic fluid behaviors. Further, the techniques utilized in this work to quantify $\mathrm{pH}$ gradients may be adapted to other microfluidic systems to ascertain the impact of Faradaic reactions on ACEO pumps or related hydrodynamic phenomena. 


\section{CHAPTER 5. STUDY OF ION CONCENTRATION CHANGE IN SPATIALLY NON-UNIFORM AC ELECTRIC FIELDS ${ }^{3}$}

\subsection{Introduction}

Alternating current (AC) electric fields applied to electrolyte fluids and/or suspended particles can induce fluid and particle movements, i.e. AC electrokinesis. AC electrokinetics is widely applied in microfluidic systems to manipulate (bio) particles and (bio) fluidics through electrophoresis (EP) [29], dielectrophoresis (DEP) [32], electroosmosis (EO) [36] and electrothermal (ET) [43]. Ideal AC electrokinesis assumptions have previously included small Faradaic currents as well as equal and opposite ion migration oscillations, it has been assumed that uniform medium conditions $(\mathrm{pH}$, ionic concentration and ionic strength) remain constant and spatially uniform throughout most experiments [7].

Despite constant medium assumptions, medium non-uniformities can arise leading to both desired and undesired phenomena. Ren et al. [158] used $\mathrm{AC}$ potentials to deflect the interface between electrolytes at different concentrations; they observed higher conductivity electrolytes tend to replace lower conductivity fluids. Concentration gradients served as a tool to control cell movements and facilitate assay development [159]. However, undesired side effects also arose: Ramos et al. [158] reported ACEO flow reversal induced by $\mathrm{pH}$ gradients. Gagnon et al. observed frequency dependent fluid displacement at liquid-liquid interface induced

3 This chapter is going to be submitted to a journal for publication. 
by AC polarization [160]. An and Minerick [108] also reported cellular osmolarity changes induced by ion concentration gradients.

Within bulk solutions, solution property non-uniformities have been studied including solution $\mathrm{pH}$ behaviors. Gencoglu et al. [130] detected $\mathrm{pH}$ gradients in an insulating DEP system. Ramos et al. [146] detected $\mathrm{pH}$ gradients in ACEO devices and further that they were a determining factor for ACEO flow reversal. $\mathrm{Ng}$ et al. [131] further quantified $\mathrm{pH}$ changes in uniform electric fields. An and Minerick [7] also detected $\mathrm{pH}$ changes and spatial $\mathrm{pH}$ gradients in non-uniform DEP fields. Such $\mathrm{pH}$ gradients were induced by Faradaic reactions at electrode surfaces.

In addition, electrolyte ion concentration changes have been studied. Khair and Squires [161] reported that charge concentration changes on wall surfaces over a nanometer scale can affect millimeter scale ion distributions under DC fields. Chang et al. [162] reported ion concentration and depletion under low frequency $\mathrm{AC}$ electric fields. In our previous work [108], micron scale fluid tonicity changes were induced by ion concentration gradients under non-uniform high frequency AC fields . Further, such phenomena were attributed ion transfer instead of Faradaic reaction according to results from multiple control experiments. In this work, we experimentally verify the existence of ion concentration gradients resulting from non-zero time averaged ion migration under non-uniform high frequency AC fields. In the following part of this paper, the non-zero time averaged ion migration is termed biased migration. 
Ion concentration changes are typically induced by ion fluxes, which can be induced by convectional flows and gradients of electrochemical potential including electric potential and chemical concentration gradients [144], described by the well know Nernst-Planck equation as follows:

$$
\boldsymbol{J}_{\boldsymbol{i}}=-D_{i} \nabla C_{i}-\frac{z_{i} F}{R T} D_{i} C_{i} \nabla \emptyset+C_{i} \boldsymbol{v}
$$

where, $\boldsymbol{J}_{i}, D_{i}, C_{i}$ and $z_{i}$ are the flux, diffusion coefficient, concentration and valence of species i, respectively. $\mathrm{R}$ is gas constant, $\mathrm{T}$ is temperature, $\mathrm{F}$ is Faraday's constant, $\emptyset$ is electric potential and $\boldsymbol{v}$ is convectional flow velocity. Combine the Nernst-Planck equation and mass conservation equation leads to the equation describing ion concentration changes:

$$
\frac{\partial C_{i}}{\partial \boldsymbol{t}}=D_{i} \nabla^{2} C_{i}+\frac{z_{i} F}{R T} D_{i} C_{i} \nabla^{2} \emptyset-C_{i} \boldsymbol{v}+R_{i}
$$

where, $\mathrm{t}$ is time and $R_{i}$ is the reaction rate for species i. From Eqn (2), time evolution of ion concentration gradients can be induced by electric potential gradients, convectional flows and ion chemical and Faradaic reactions.

In this work, ionized fluorescent FITC dye was used to visualize ion concentration gradients induced by electromigration. Fluorescing ion concentration was measured via spatial emission intensity. Since most ionizable dyes are also $\mathrm{pH}$ sensitive, two control strategies were implemented to isolate electromigration from Faradaic reaction effects. This is important because Faradaic reactions govern water electrolysis and thus $\mathrm{H}+$ generation in microfluidic systems. First, methanol $(\mathrm{MeOH})$ was used as an inert solvent that inhibits electrolysis generating negligible $\left[\mathrm{H}^{+}\right]$. Second, a Hafnium Oxide $\left(\mathrm{HfO}_{2}\right)$ dielectric layer was electrodeposited to 
prevent contact between the metallic electrodes and electrolyte solution. Electric fields can still tunnel through the dielectric layer, but electron transfer between the electrodes and electrolyte was blocked to prevent Faradaic reactions at the electrode surface.

Three novel strategies were utilized compared with traditional electrolyte solutions. First, this work utilizes different targeting ions by directly detecting ionized dye concentrations as opposed to utilizing electrolyte ion concentrations (such as $\mathrm{K}^{+}, \mathrm{Na}^{+}$or $\mathrm{Cl}^{-}$). Second, dilute concentrations of $2 \times 10^{-6} M$ ionized dye are much lower than electrolyte concentrations in traditional buffer solutions. Third, the solution relative permittivity differs greatly when using $\mathrm{MeOH}(\varepsilon=33)$ instead of water $(\varepsilon=78)$. To compare results to previous works and applicable for future applications, a non-dimensionalized frequency was obtained by dividing the applied frequency by the electrode charging frequency [84]:

$$
\begin{array}{r}
f_{c}=\frac{D}{\lambda_{D} L} \\
\lambda_{D}=\sqrt{\frac{\varepsilon R T}{2 F^{2} C_{0}}} \\
f_{r}=\frac{f}{f_{c}}
\end{array}
$$

Where, $f_{c}$ is electrode charging frequency, $D$ is diffusion coefficient, $\lambda_{D}$ is electrical double layer thickness, $L$ is the characteristic length of the system, $\varepsilon$ is relative permittivity of solution, $R$ is gas constant, $T$ is temperature, $F$ is Faradaic constant, $C_{0}$ is bulk molar concentration, $f_{r}$ is relative frequency and $f$ is applied frequency. Under our current experimental conditions $\left(2 \times 10^{-6} M\right.$ FITC in $\left.\mathrm{MeOH}\right)$, with the smallest 
characteristic length of $100 \mu \mathrm{m}$, the electrode charging frequency was calculated to be $\sim 35 \mathrm{~Hz}$.

Qualitative control experiments were designed to exclude emission intensity impacts from Faradaic reactions and convectional flow. Thus, detected emission intensity changes are induced by ion concentration gradient. Time analysis was then performed on intensity behaviors and the ion concentration gradient was inspected using contour plots. Finally, we detect the ion concentration gradient at potentials from 5 to $10 \mathrm{~V}_{\mathrm{pp}}$ and frequencies from $3 f_{c}$ to $15 f_{c}$.

\subsection{Materials and Methods}

\subsubsection{Materials}

Fluorescein isothiocyanate (FITC, 492/518 nm) was utilized as a fluorescing ion source. FITC (Sigma-Aldrich, USA) solution was prepared in $\mathrm{MeOH}$ (99.99\%, Sigma-Aldrich, USA) at a stock concentration of $10^{-4} \mathrm{M}$.

Rhodamine B ( $\mathrm{Rb}, 554 / 627 \mathrm{~nm}$ ) was employed as a neutral dye in control experiments. Rb (Sigma-Aldrich, USA) solution was also prepared in $\mathrm{MeOH}$ at $10^{-4} \mathrm{M}$.

$\mathrm{NaCl}(>99 \%$, Macron Chemicals, USA) $\mathrm{MeOH}$ solution at the same solution conductivity as FITC $(0.00043 \mathrm{~S} / \mathrm{m})$ was utilized during the $\mathrm{Rb}$ control experiment to induce similar flow patterns. 


\subsubsection{Microdevices Design and Fabrication}

Orthogonally located Ti/Au (50/50 nm thickness) electrode pairs with $100 \mu \mathrm{m}$ gap were designed and fabricated to provide spatially non-uniform electric fields following standard soft photolithography fabrication procedures [108]. A $100 \mathrm{~nm}$ layer of Hafnium Oxide $\left(\mathrm{HfO}_{2}\right)$ was coated over the entire glass slide to prevent contact between the electrode and electrolyte solution. $\mathrm{HfO}_{2}$ was deposited via electrosputtering (Perkin Elmer, USA) for $40 \mathrm{~min}$ at $100 \mathrm{~V}$ biased target voltage. Fig. 5.1 shows a a) photo of

the intact device, b) magnified view of experimental chamber with electrodes outlined and chamber edges noted, and c) device side view to illustrate the dielectric $\mathrm{HfO}_{2}$ layer configuration. An Agilent waveform generator 33250A supplied the necessary AC electric signal across the vertical electrode $\left(\mathrm{V}_{\mathrm{pp}} \sin (\omega \mathrm{t})\right)$ and horizontal electrode (ground) to drive the ion migration in the chamber.

\subsubsection{Experiments}

FITC and Rb photobleaching were independently quantified over $60 \mathrm{~s}$ of constant excitation light exposure by continuously detecting emission light intensity in the chamber. No electric fields were supplied during this control test. 
a)

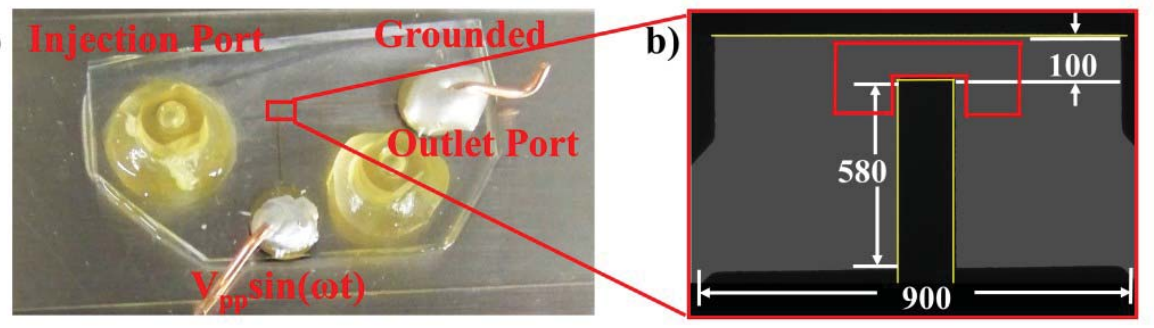

c)

d)
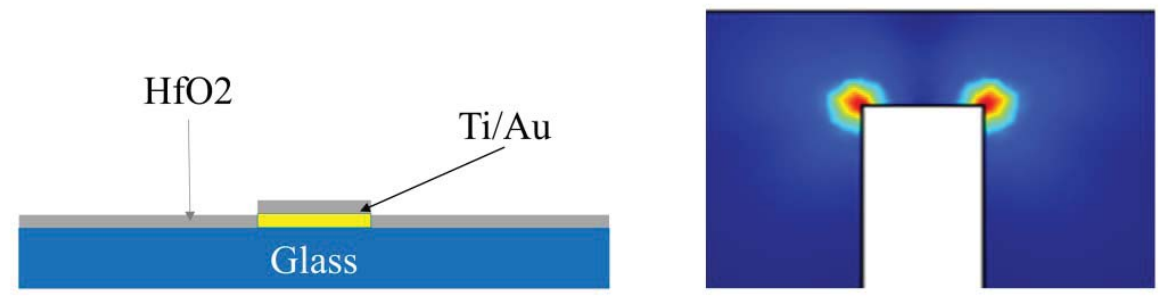

Figure 26: Microdevice design. a): Photo of device, with fluid channel filled with green dye, red boxes the working fluidic chamber and $b$ ): Red box area under 10x microscope, c): Side view of the device. Units for d): COMSOL simulation of the gradient of electric field in labeled area in Fig. 26b. All numbers are micrometers.

Experimental samples were prepared from stock FITC solutions and $\mathrm{MeOH}$ at a 1:49 ratio. Ion concentration experiments utilized $0.002 \mathrm{mM}$ FITC in $\mathrm{MeOH}$ while control experiments utilized mixtures of $\mathrm{Rb}$ in $\mathrm{MeOH}$ and $\mathrm{NaCl} \mathrm{MeOH}$ with final $\mathrm{Rb}$ concentration of $0.002 \mathrm{mM}$. Experimental samples were injected into the input port shown in Fig. 26 (a) then sealed with one-piece fittings (Labsmith, USA) to create a batch system free from any net pressure-driven flow. The device was mounted on the microscope stage and AC signals ranging in amplitude from 5 to $10 \mathrm{~V}_{\mathrm{pp}}$ and frequencies from 100 to $500 \mathrm{~Hz}$ were applied. According to our experimental conditions $\left(2 \times 10^{-6} \mathrm{M}\right.$ FITC in $\left.\mathrm{MeOH}\right)$, the electrode charging frequency was around $35 \mathrm{~Hz}$, thus the applied 
non-dimension frequency $f_{r}$ ranges from 3 to 12 . Microscope videos at 10x magnification were recorded at $1 \mathrm{fps}$ for 60 seconds; the first 5 frames were without electric field application to check system stability. Each experiment was repeated 5 times.

To observe Faradaic reaction contributions, parallel electrode pairs providing uniform electric fields at $100 \mathrm{~Hz}, 10 \mathrm{~V}_{\mathrm{pp}}$ were also studied.

\subsubsection{Data Analysis Methods}

Fluorescent image intensity analysis was processed in 2-D grayscale. Videos were exported as 61 (0-60 s) figures and imported into MATLAB as grayscale matrixes ranging from 0 (dark) to 255 (bright). The fluidic region intensity was standardized to 75 to enable comparisons between experiments and ensure all pixels remained within the 255 maximum without scaling. Effects due to photobleaching were corrected via Fig. 27(c)'s equation. To better visual changes, the MATLAB Image Processing toolbox generated 3-D mesh images with the z-axis expressing emission intensity and contour plots to show intensity profile gradients. 

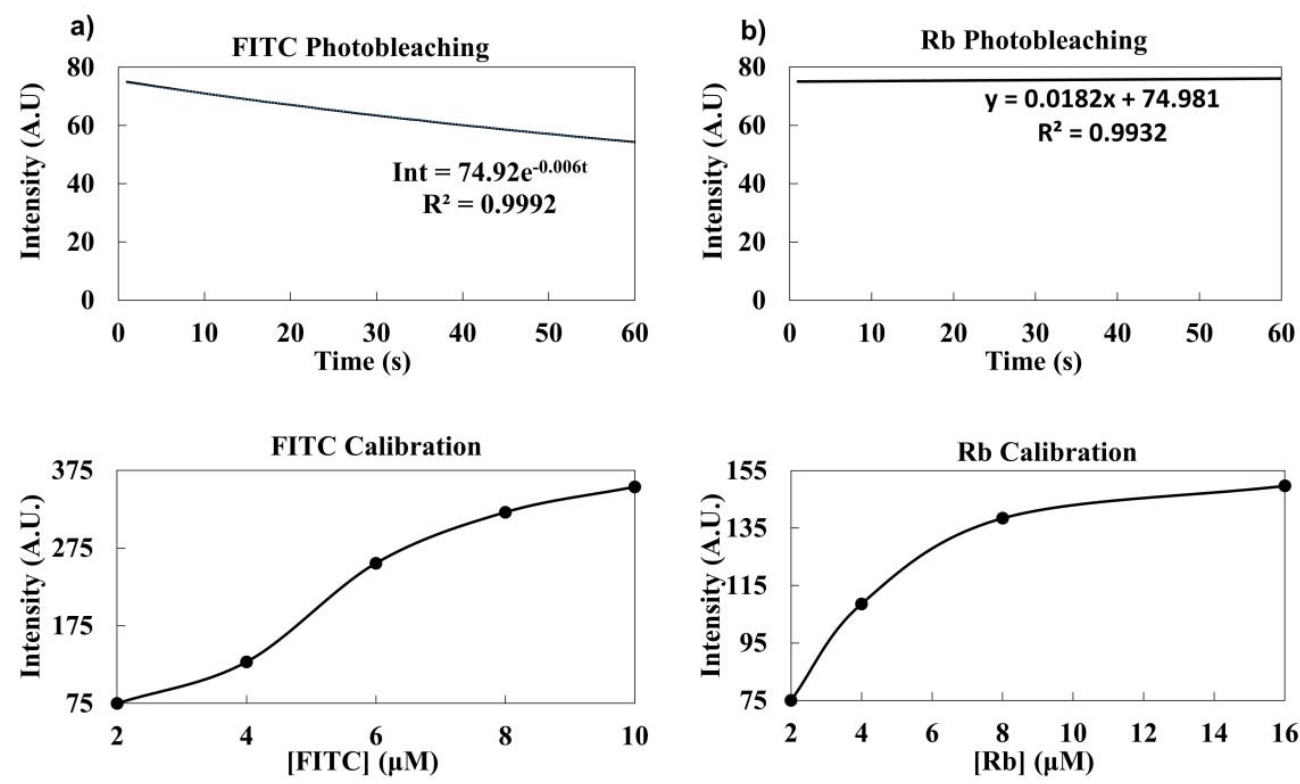

Figure 27: Dye properties study a): Photobleaching property for FITC; b) Photobleaching property in for Rb; c) FITC emission intensity calibrated with FITC concentration and d) Rb emission intensity calibrated with $\mathrm{Rb}$ concentration

Peak-to-peak potential and frequency dependency analyses were performed using ImageJ. Areas of interest were selecting according to emission intensity gradients and individually examined. The high field density area was chosen for subsequent analysis since DEP and induced flow phenomena was most pronounced in this region.

\subsection{Results and Discussion}

In order to observe ionic concentration gradients, an anionic fluorescent FITC molecule was detected as a function of time, position, geometry, applied potential, and frequency. To determine photobleaching effects, a calibration curve between FITC emission intensity and FITC 
concentration was generated. Control experiments were first performed to observe Faradaic reaction contributions and effects from convectional flow; experimental conditions were strategically selected to minimize electric double layer formation and thus these effects. Spatial intensity and time analyses were performed to track ion concentration changes in electric fields. Finally, signal magnitude and frequency dependence were quantified to determine ion migration trends in the oscillated $\mathrm{AC}$ electric fields.

\subsubsection{FITC Calibrations}

Ionized FITC and non-ionized Rb controls were completed to account for both photobleaching, hydrodynamics, and baseline intensity calibrations. Photobleaching properties were examined by detecting FITC (Fig. 27 a) and Rb (Fig. 27 b) emission intensity without applying electric fields but under the same exposure regiment. Result showed that FITC emission intensity decreased from 75 (A.U.) to 55 while $\mathrm{Rb}$ emission intensity remained unchanged over $60 \mathrm{~s}$. FITC photobleaching can be fit in exponential function and $\mathrm{Rb}$ photobleaching can be fit in linear function with both functions having $\mathrm{R}^{2}>0.993$ for future use to obtain net intensity change induced by dye concentration change.

Second, both FITC and Rb concentration was calibrated with emission intensity as shown in Fig. 27(c,d). Both FITC and Rb emission intensity increase with dye concentration from $2 \mu \mathrm{M}$ to $12 \mu \mathrm{M}$. In our experiments, FITC concentration ([FITC]) is 
obtained using the obtained polynomial fit with obtained $\mathrm{R}^{2}>0.9999$.

\subsubsection{Control Experiments}

Control experiments were conducted to elucidate other potential mechanisms inducing emission intensity change. According to equation 2, there are diffusion, reaction, convection and electromigration which would impact on ion concentration change with time. Diffusion is a passive process that always exist in all systems. Faradaic reaction based on charge transfer needs to be inspected since such reaction would affect system $\mathrm{pH}[7,8]$ thus affect dye emission intensity. For convectional flow, AC electroosmosis flow is the most possible induced phenomenon under experimental conditions in this work and the induced flow might farther affect dye emission intensity by affecting dye concentration. As a result, convectional flow also needs to be inspected.

The dielectric properties of Hafnium Oxide $\left(\mathrm{HfO}_{2}\right)$ such as [163]: Dielectric constant=18.7, Dielectric breakdown potential $=34 \mathrm{~V}$, Breakdown field $=5.4 \mathrm{MV} / \mathrm{cm}$ and High resistivity would provide us a good insulating layer between electrode and electrolyte while still providing electric fields strong enough for ion migration under our experimental condition. Also, $\mathrm{MeOH}$ is known as a much more inertia solvent than water to resist electrolysis [152]. 
To verify the effect from Faradaic reaction, FITC solution at conductivity of $4.3 \times 10^{-4} \mathrm{~S} / \mathrm{m}$ was detected in parallel electrode pair designed to provide uniform electric fields thus the gradient of electric fields $\nabla^{2} \emptyset$ is zero thus under AC electric fields there should not be time averaged ion migration or induced flow phenomena.

To verify the effect from induced flow, mixture of neutral dye $\mathrm{Rb}$ and $4.3 \times 10^{-4} \mathrm{~S} / \mathrm{m} \mathrm{NaCl}$ solution was used to detect behavior of neutral molecule that was not affected by electromigration under conditions having similar induced flow. Experiments were taken under both parallel (uniform fields) and orthogonal (non-uniform fields) electrode pair.

$10 \mathrm{~V}_{\mathrm{pp}} 100 \mathrm{~Hz}$ sinusoidal signal was applied on all of four sets of control experiments. Fig. 28 shows the results from control experiments that have already corrected for photobleaching. Results at $\mathrm{t}=0,7$ ( $2 \mathrm{~s}$ after applying electric fields) and $60 \mathrm{~s}$ are shown in Fig. 28(a-d), 3 (e-h) and 5.3(i-1). Fig. 28(m-p) show the difference between $60 \mathrm{~s}$ and $0 \mathrm{~s}$ to facilitate a view of intensity change through the experiment. In those, Fig. 28(m, n, p) are showing intensity difference obtained from $\Delta I=I_{t=60}-I_{t=7}$ while Fig. 28(n) is showing negative intensity difference obtained by $\Delta I=I_{t=7}-I_{t=60}$ due to the observation of intensity decrease attributed to remaining Faradaic reaction. Further explanation of 
this observed intensity decrease will be explained in section 3.3.
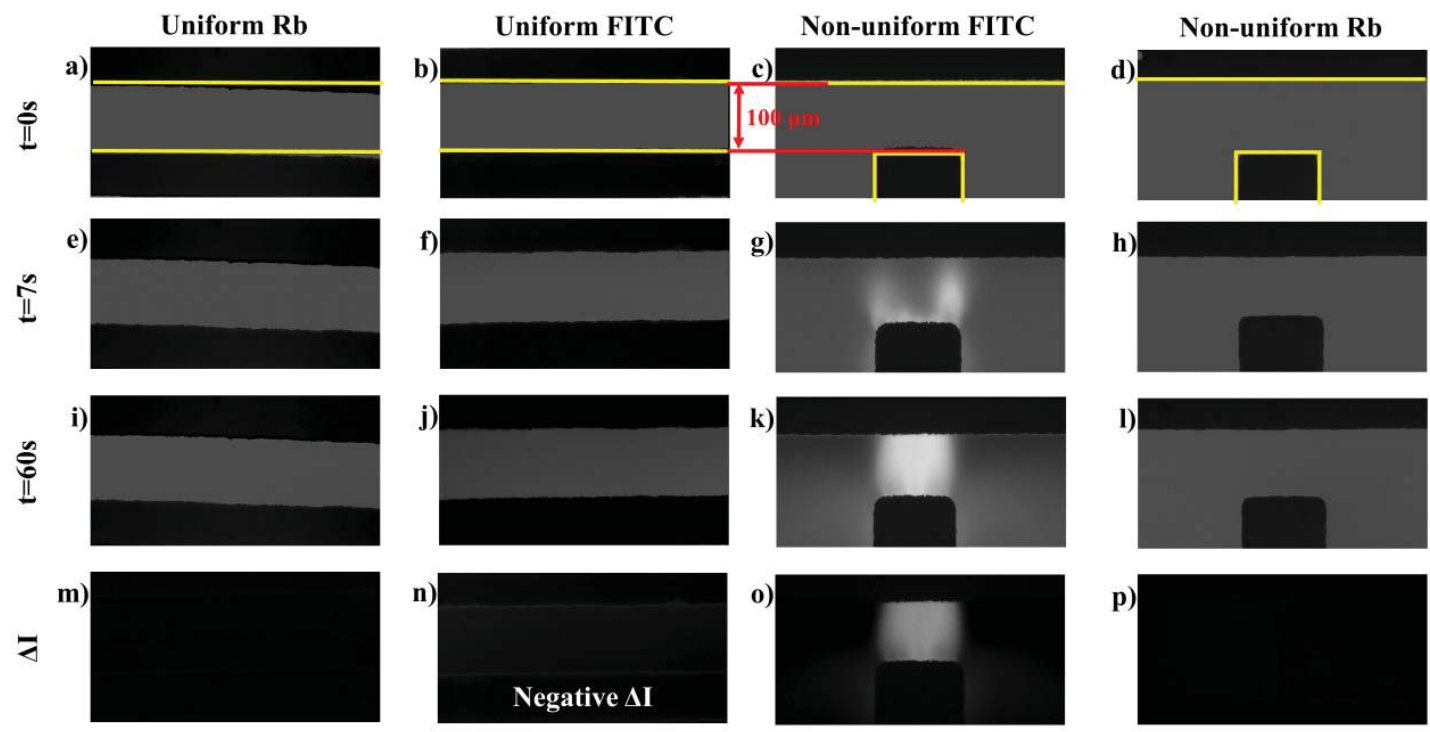

Figure 28: Comparison experiments under $100 \mathrm{~Hz}, 10 \mathrm{Vpp}$; First column are $\mathrm{t}=0,7,60 \mathrm{~s}$ and FITC intensity difference obtained by MATLAB image analysis of experiments under uniform electric field; Second column are same time and image output of $\mathrm{Rb}$ intensity; Third column are same time and image output of experiments under nonuniform electric field for ionized FITC; Fourth column are same information of experiments using non-ionized $\mathrm{Rb}$ dye in non-uniform electric field It can be observed that intensity increase can be observed in o) while no change observed $m$ ) $n$ ) and $p$ )

For $\mathrm{Rb}-\mathrm{NaCl}$ solution in uniform electric fields generated by parallel electrode pair, the emission intensity shown in Fig. 28(a, e,

i) stays almost unchanged. Neither positive nor negative intensity difference can be observed. This indicates that there is no significant temperature change in the system due to the low conductivity of solution (estimated temperature change would be $\sim 0.01^{\circ} \mathrm{C}$ according to $\Delta T=\frac{\sigma V_{r m s}^{2}}{8 k}$, where $\sigma=0.00043$ is the solution conductivity and $\mathrm{k}$ is thermal conductivity, for 
$\mathrm{MeOH}=0.203$ at $\left.25^{\circ} \mathrm{C}\right)$.

For FITC solution in uniform electric fields, the emission intensity shown in Fig. 28(b, f, j) stays almost unchanged. A small negative intensity change with average magnitude of 7.5 in the bulk area (A.U.) was observed in Fig. 28(n) indicating an intensity decrease from $\mathrm{t}=7$ and $60 \mathrm{~s}$. This observation of slight intensity decrease is attributed to the remaining Faradaic reaction taking place between the water contamination in $\mathrm{MeOH}$ [164] and leftover exposed Au electrode during the photoresist lift-off process [165]. However, such Faradaic reaction mechanism is only inducing minor and uniform intensity decrease indicating the Faradaic reaction is not the mechanism inducing emission intensity increase in the system.

For FITC solution in non-uniform electric fields, the system started from uniform intensity as shown in Fig. 28(c). Fig. 28(g) shows the emission intensity started to increase from the high field density area shown in Fig. 26(d) after $2 \mathrm{~s}$ application of electric field. In Fig. 28(k) and Fig. 28(o), significant intensity increase with average magnitude of 120 can be observed in the central area of the system at long time range of $60 \mathrm{~s}$ and long length scale of $100 \mu \mathrm{m}$.

For $\mathrm{Rb}-\mathrm{NaCl}$ solution under non-uniform electric fields, no significant intensity change was observed from Fig. 28(d, h, l) and 
the averaged magnitude of intensity change in bulk area shown in Fig. 28(p) is close to 0 . Since similar conductivity as FITC solution was provided using $\mathrm{NaCl}$, similar ACEO should be induced. As a result, the negligible intensity increase indicates that the induced ACEO flow is not affecting the neutral species concentration in the system thus convectional flow is not the mechanism inducing the observed emission intensity in FITC solution under non-uniform electric field.

According to equation 2, these comparison experiments suggest the observed FITC intensity changes is not induced by neither the reaction or convection term of the transport equation nor any other potential effects affecting the FITC emission intensity such as temperature or electric field itself. This is indicating that the observed intensity change is induced by electromigration under non-uniform electric field.

\subsubsection{Proposed Mechanism for the Established Ion Concentration Gradient}

It has been proved in section 3.2 that the observed intensity increase is due to [FITC] enrichment. However, the mechanism for the establishment of ion concentration gradient is still ambiguous, especially under the impact from $\mathrm{AC}$ electric field instead of DC, where ions are traditionally recognized to experience oscillated migration with the applied signal resulting in minor solution property change. 
Equation 2 is describing the ion change to time with respect to the combination of ion transport and mass conservation. It has been proved that the effect from convectional flow is negligible, $C_{i} \boldsymbol{v}=\mathbf{0}$. Then, if the system is under a quasiequilibrium condition, where the time averaged change of ion concentration with time is zero, equation 2 can be turned into following form:

$$
-D_{i} \nabla^{2} C_{i}=\frac{z_{i} F}{R T} D_{i} C_{i} \nabla^{2} \emptyset+R_{i}
$$

The left hand side of equation 7 is the diffusion term related to concentration gradient. The first term at right hand is describing effects of electromigration and the reaction term was included since the minor reaction of water electrolysis has potential to affect the emission intensity although it is not directly influencing the ion concentration. Equation 7 implies a non-zero term for the gradient of electric field (Laplacian of potential) is necessary for the formation of ion concentration gradient. 

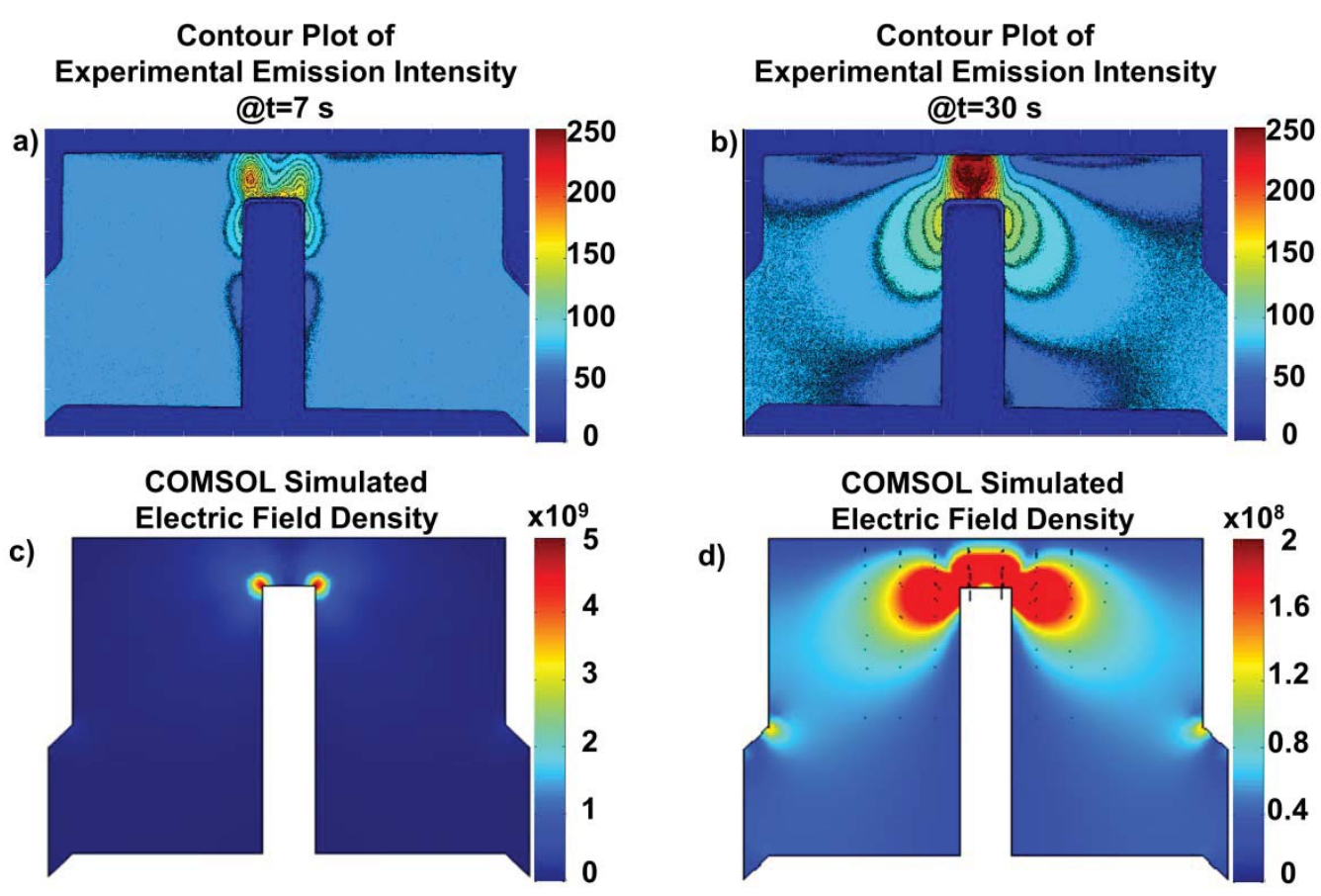

Figure 29: Comparison between contour plot of experimental emission intensity at $\mathrm{t}=7$ and $30 \mathrm{~s}(\mathrm{a}, \mathrm{b})$ and COMSOL simulated electric field density represented at different color scales $(c, d)$.

Firstly, such explanation matches to the control experimental results: in uniform electric field, where $\nabla^{2} \varnothing=0$, no ion concentration gradient was established while in non-uniform electric field, the non-zero gradient of electric field shown in Fig. 26(d) rises the ion concentration gradient. Nevertheless, the similarity between pattern of established ion concentration gradient and electric field density is also supporting this explanation: Fig. 29 shows the comparison between the contour plot for emission intensity indicating ion concentration distribution at $\mathrm{t}=7$, and $30 \mathrm{~s}$ in Fig. 29(a,b) and the electric field density simulated by COMSOL in Fig. 29(c,d). The two field density plots are showing the same data with different scale for best visualization: Fig. 29(c) is showing the data in the 
scale from $1 \times 10^{8}$ to $5 \times 10^{9} \mathrm{~V} / \mathrm{m}^{2}$ while Fig. 29(d) is showing data in the scale from $4 \times 10^{5}$ to $2 \times 10^{8} \mathrm{~V} / \mathrm{m}^{2}$. Matching of the pattern between Fig. 29(a) and (c) is showing that the ions start to enrich from the highest field density area in the system. With time past, the ion enrichment magnitude increased and the enrichment area is also expanding to the areas with second highest field densities according to the comparison between (b) and (d). Our previous work [] on detecting $\mathrm{pH}$ change showed the Faradaic reaction generated $\left[\mathrm{H}^{+}\right]$that decreases the emission intensity gives the highest magnitude around the tip of vertical electrode, which can explain the pattern difference in the corner area around the vertical electrode between Fig. 29(b) and (d). Also, ion and bulk solution replenishment between the connection channel and bulk solution is providing extra ions from connection channels and conductivity induced flux is also causing differences between the ion distribution and electric field density distribution, especially in area B and C. Such replenishment will be discussed further in section 3.4. As a result, it can be concluded that the establishment of ion concentration gradient is due to the existence of non-zero electric field density induced by spatial non-uniformity of electric field, which is introducing a ion biased directional migration from lower field density area to higher field density area between each half cycle of oscillation.

\subsubsection{Spatial and Time analysis of Intensity Change}

Since the intensity change is induced by ion migration, further time and spatial analysis was performed for understanding the physical mechanism and process for the formation of such ion concentration gradient. Fig. 30 shows 
experimental results at $10 \mathrm{~V}_{\mathrm{pp}}$ and $100 \mathrm{~Hz}$ after photobleaching corrections such that intensity increases should be only caused by ion migration. The photobleaching corrected data was then fit to the calibration curve shown in Fig. 27(c) to obtain [FITC]. Results are organized into columns corresponding to experiment time: column 1 is for $\mathrm{t}=0(\mathrm{a}, \mathrm{e}, \mathrm{i})$, column 2 is for $\mathrm{t}=7 \mathrm{~s}(2 \mathrm{~s}$ after applied electric field), column 3 is for $\mathrm{t}=30 \mathrm{~s}(\mathrm{c}, \mathrm{g}, \mathrm{k})$ and column 4 is for $\mathrm{t}=60$ $\mathrm{s}(\mathrm{d}, \mathrm{h}, \mathrm{l})$. The respective rows from top to bottom are: first row shows 2-D grayscale intensity image reformed by Matlab (a-d), the second row is a 3-D mesh image from Matlab where the $\mathrm{z}$ axis demonstrates the [FITC] (e-h), and the third row is a contour plot to facilitate the view on the gradient of [FITC] $(\nabla[$ FITC $])$ in the system (i-1). Fig. 30(m) shows the areas from which averaged [FITC] was obtained by ImageJ. The areas were chosen according to the contour plot shown in Fig. 30(1). Area A shows the highest emission intensity thus highest [FITC] during the entire experiment. Area B is the area surrounding the tip of vertical electrode. Area $\mathrm{C}$ is the bulk solution area, where intensity thus [FITC] stayed mostly unchanged. Area D and E are corner areas where shows lower emission intensity attributed to [FITC] depletion according to the contour plot Fig. 30(1). The inset in Fig. 30(m) shows the interest area $\mathrm{F}$, where the electric field density is strongest thus greatest DEP and induced flow phenomenon exists in such area. The area 'AA' is the whole area inspected in the system. Fig. 30(n) shows the average of area [FITC] as a function of time. 

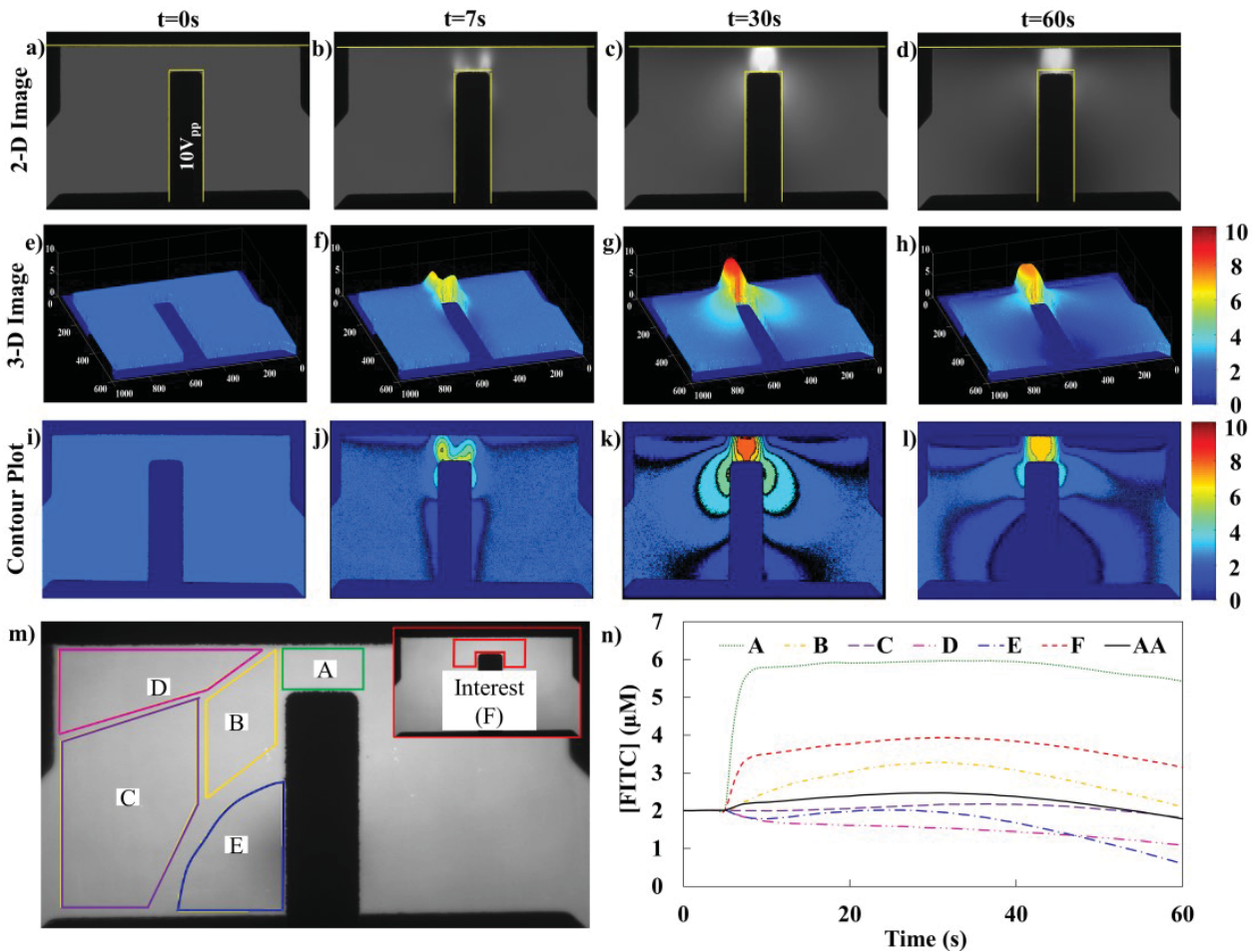

Figure 30: Ion concentration time analysis: a)-d): Gray-scale 2-D dye intensity plot obtained from MATLAB image analysis at $\mathrm{t}=0,7,30$ and 60 s under 20Vpp $100 \mathrm{~Hz}$; e)-h): 3-D MATLAB plot at same time point and i)-1): Matlab contour plot showing the dye intensity gradient. Yellow box in figure $\mathrm{m}$ ) shows the position of sampled intensity. Figure $n$ ) is showing the time dependency of the average of sampled intensity.

For the first column at $\mathrm{t}=0$, plots demonstrate the uniform intensity where no electric field was applied; for subsequent analysis, this initial $\mathrm{c}$ is always normalized to 75 (A.U.) in order to keep the highest intensity after application of electric field fall into the range of 0-255 for MATLAB image analysis. These plots show the uniform [FITC] distribution in the microdevice system without influence from electric field.

The electric potential was applied at $\mathrm{t}=5 \mathrm{~s}$. At $\mathrm{t}=7 \mathrm{~s}$ after 2 seconds of the impact from electric field, slightly higher [FITC] around $6 \mu \mathrm{M}$ was observed in 
area A shown in Fig. 30(m) while [FITC] in other areas stayed almost unchanged according to Fig. 30(f) and 5.5(j) except a small decrease in corner area $\mathrm{D}$ and $\mathrm{E}$ and their mirror symmetric areas. A very interesting observation is that the pattern of such [FITC] enrichment at short time range $(<2 \mathrm{~s})$ matches the electric field density distribution well shown in Fig. 26(d).

Such observation of [FITC] enrichment and depletion indicates that at short time range of $2 \mathrm{~s}$ or during the first 200 cycles of oscillation, FITC ions started to enrich in high electric field density areas. Since it has been proved that such enrichment was induced by electromigration, the depletion can be attributed to mass conservation - the FITC ions migrated from the depleted area to the enriched area and there is not enough time for diffusion to replenish the depleted area yet in 2 seconds from the bulk area $\mathrm{C}$ due to the low diffusivity of FITC $\left(D=4.9 \times 10^{-10} \mathrm{~m}^{2} / \mathrm{s}, t \approx \frac{x^{2}}{2 D}=10 \mathrm{~s}\right.$ for $\mathrm{x}=100$ $\mu \mathrm{m}$ where, $\mathrm{D}$ is diffusion coefficient, $\mathrm{x}$ is diffusion length).

At $\mathrm{t}=30 \mathrm{~s}$, significant [FITC] up to $10 \mu \mathrm{M}$ is detected in area A. In peripheral areas (area B and its mirror symmetric area), moderate [FITC] around $4.5 \mu \mathrm{M}$ can be also observed with the enrichment magnitude decrease with the increase of distance from the high field density area. However, in corner areas (area $\mathrm{C}$ and $\mathrm{D}$ and their mirror symmetric areas), the observed [FITC] is around 0.5 to $1 \mu \mathrm{M}$, which is lower than the initial [FITC] of 2 implying a FITC ion depletion.

It was observed in Fig. 28(j) that the [FITC] enrichment initiates in the high field density area at the left and right corner on the tip of horizontal 
electrode. In Fig. 28(k), the highest [FITC] area expanded into center part of area A. This can be understood as following: the induced [FITC] enrichment is initiated in higher field density area as shown in Fig. 28(j) due to the effect of electromigration. Such enrichment is introducing both ion concentration gradient and solution conductivity gradient. Once such ion and conductivity gradient established, ion diffusion and conductivity gradient induced flow $[166,167]$ is existed in the system. Ion diffusion is due to the chemical potential gradient induced by ion concentration gradient with the direction from higher concentration to lower concentration due to the nature of entropy. Electrolyte with higher conductivity is also reported to replace electrolyte with lower conductivity under the effect of electric field [158] and in this system, the ion concentration gradient is providing such conductivity difference thus higher concentration electrolyte with higher conductivity is tend to move toward and replace the lower concentration electrolyte with lower conductivity. As a result, both the FITC ions and bulk solutions with higher conductivity tend to move from the higher field density area to both the central area and the peripheral areas while at the same time, the electromigration phenomena is still attracting the ions from lower field density peripheral areas into the higher field density area. In short summary, the ions in bulk areas with lower field density $(C, D, E)$ migrates to the higher field density area and establish high concentration then diffuse or flow outward due to the ion and conductivity concentration, resulting in the observed [FITC] distribution shown in Fig. 30(k) with the magnitude decrease with the increase of distance from higher field density area. As a result, [FITC] depletion exists in area C, D and E. However, in area $\mathrm{C}$, once the ion depletion occurred, ion concentration gradient and conductivity gradient was established between area $\mathrm{C}$ and the connection 
channels at the left and right edge of the figure, which is connecting to input and output ports thus should be regarded as solution sources with initial $2 \mu \mathrm{M}$ solution. Due to this ion and conductivity gradient, solution in connection channels tend to replenish the [FITC] depletion in the area C. For area D and $\mathrm{E}$ in corner areas in the system far away from the connection channel, less replenishment is provided, resulting in the observation of [FITC] depletion.

At $\mathrm{t}=60 \mathrm{~s}$, while [FITC] enrichment still exists in area A, the magnitude is decreased from $\sim 10 \mu \mathrm{M}$ at $\mathrm{t}=30 \mathrm{~s}$ to $\sim 7 \mu \mathrm{M}$. Also, in peripheral areas (area $\mathrm{B}$ and its mirror symmetric area), the area with higher [FITC] shrunk and at corner areas (area C and D and their mirror symmetric areas), lower [FITC] down to 0 to $0.5 \mu \mathrm{M}$ is observed, implying a further FITC ion depletion.

Such observation was made due to the competition between 1): the same combined effect of electromigration, diffusion, induced flow and replenishment discussed for $\mathrm{t}=30 \mathrm{~s}$ and 2): the Faradaic reaction due to the possible existence of water molecule introduced by air contamination and the exposed gold electrode during the lift-off process observed by the uniform electric field control experiment shown in Fig. 28(n). From $t=30 \mathrm{~s}$ to $\mathrm{t}=60 \mathrm{~s}$, FITC ions still tend to migrate from lower field density areas $(C, D, E)$ into higher field density areas (A, B), which induced further [FITC] depletion in the corner areas $\mathrm{D}$ and $\mathrm{E}$ where the replenishment effect from connection channel is minimum while in area $\mathrm{C}$ such replenishment effect is stronger thus lower magnitude of [FITC] decrease is observed. However, the observed emission intensity also decreases from up to 253 to around 230 in central area A and the surrounding area B despite the fact of more ions are supposed to be 
migrated into these areas. Such unexpected observation is attributed to the remaining Faradaic reaction. In our system containing FITC, MeOH and small amount of water molecule introduced by possible contamination, there is potential for Faradaic reaction generating small amount of $\mathrm{H}^{+}$which is decreasing emission intensity. Our previous work [] has studied this Faradaic reaction in non-uniform $\mathrm{AC}$ electric field thoroughly. Generally, under the condition of uniform conductivity and electrolyte concentration, higher $\left[\mathrm{H}^{+}\right]$ was produced in the interest area $\mathrm{F}$ at $\mathrm{t}=60 \mathrm{~s}$ while the $\left[\mathrm{H}^{+}\right]$in the whole system did also increase with the magnitude decreased with the increase of distance from area F. In this work, due to the introduction of $\nabla[$ FITC $]$ and conductivity gradient, especially in area $\mathrm{A}$, greater higher $\left[\mathrm{H}^{+}\right]$production is expected in area A thus higher intensity decrease from 253 to 230 is observed in such area, which is greater than the intensity decrease around 7 observed in the control experiment using uniform electric field using uniform $2 \mu \mathrm{M}$ FITC solution. Also, due to the Faradaic reaction, whole system emission intensity decrease is expected, which can explain the lower intensity around 65 observed in area C. However, the effect from electromigration is still dominating over the effect from Faradaic reaction and obvious intensity increase thus ion enrichment can still be observed, although the detected intensity thus [FITC] at $60 \mathrm{~s}$ is lower than it is.

To qualify the time dependence of ion concentration by area, Fig. 30(n) compares different areas divided according to the contour analysis in Fig. 30(k). Averaged [FITC] in each area was plotted as function of time in Fig. 30(n). For area C, D and E, [FITC] show a steady value of $2 \mu \mathrm{M}$ before $5 \mathrm{~s}$, then once the electric field is applied, [FITC] depletion is observed. For area 
AA including all area in the system excluding the electrodes, [FITC] stayed almost unchanged in the first $40 \mathrm{~s}$ resulting in the observed [FITC] of 1.9 at 60 s. Area B experiences [FITC] enrichment to $3 \mu \mathrm{M}$ after 30 seconds applying the electric field then decrease to around $2.2 \mu \mathrm{M}$ at $\mathrm{t}=60 \mathrm{~s}$. Area A experiences the strongest [FITC] enrichment with a magnitude of $6 \mu \mathrm{M}$ at $\mathrm{t}=35 \mathrm{~s}$ then slightly decreased from $\mathrm{t}=40 \mathrm{~s}$ to $\mathrm{t}=60 \mathrm{~s}$ back to $5.5 \mu \mathrm{M}$. In interest area $\mathrm{F}$, where strongest DEP and induced flow are experiencing strongest effect, observed [FITC] increased up to $4 \mu \mathrm{M}$ in the first 30 seconds after applying electric field then slightly decreased to $3.2 \mu \mathrm{M}$ at $\mathrm{t}=60 \mathrm{~s}$.

Area C, D and E are experiencing [FITC] depletion since they are in low electric field density areas as discussed above. The magnitude of ion depletion in area $\mathrm{D}$ and $\mathrm{E}(1$ and $1.5 \mu \mathrm{M})$ are higher than area $\mathrm{C}(\sim 0.2)$ because area $\mathrm{D}$ and $\mathrm{E}$ are in the corner areas where ion/solution replenishment from connection channel is more difficult to reach to while area $\mathrm{C}$ is in direct contact with such replenishment channel and is kept under almost the initial condition of $2 \mu \mathrm{M}$. The almost unchanged [FITC] in the whole area AA shows great feature of the mass conservation in the system, despite the existence of minor Faradaic reaction and introduction of ions due to the replenishment effect. Area A and interest area F show significant ion enrichment before $30 \mathrm{~s}$ inducing emission intensity increase dominating over the intensity decrease induced by Faradaic reaction. However, from 30 to $60 \mathrm{~s}$, the intensity decrease induced by Faradaic reaction started to be noticeable in addition to the intensity increase by electromigration thus the observed system emission intensity decreased even if the ion concentration gradient does not actually decrease. As a result, in the following section of frequency and peak-to-peak 
inspection, the [FITC] at $\mathrm{t}=35 \mathrm{~s}$ is used instead of $\mathrm{t}=60 \mathrm{~s}$ to exclude the effect from Faradaic reaction and describe the effect of electromigration at the best extent.

\subsubsection{Peak-to-peak Potential and Frequency Dependency}

Lastly, peak-to-peak potential $\left(\mathrm{V}_{\mathrm{pp}}\right)$ and frequency dependency of [FITC] change was inspected. $\mathrm{V}_{\mathrm{pp}}$ range was chosen from $5 \mathrm{~V}$ to $10 \mathrm{~V}$ at fixed frequency of $100 \mathrm{~Hz}$ since negligible intensity increase was observed below 5 V. Frequency range was chosen from $100 \mathrm{~Hz}$ to $500 \mathrm{~Hz}$ (relative frequency=3 to 14) at fixed applied potential of $10 \mathrm{~V}_{\mathrm{pp}}$. Averaged area intensity change in interest area $\mathrm{F}$ between $\mathrm{t}=5 \mathrm{~s}$ and $35 \mathrm{~s}$ was used to represent the system intensity change thus ion concentration change. Each group of results contained 5 repeats with standard deviation labeled.
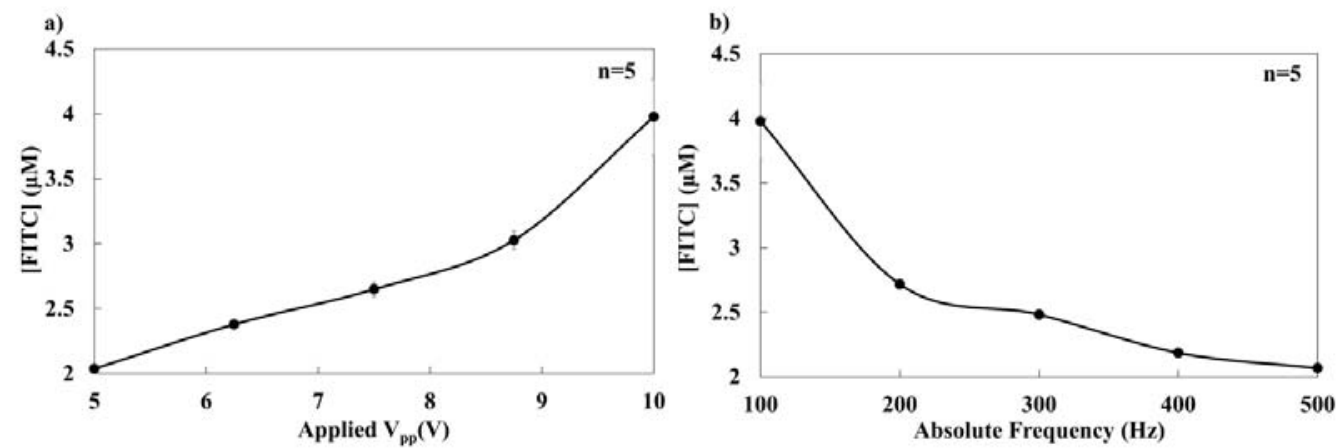

Figure 31: a) : potential dependency of intensity change at $100 \mathrm{~Hz}$ and b): frequency dependency of intensity at $10 \mathrm{Vpp}$. This shows intensity change increase with peak-to-peak potential and decrease with frequency, which implies the ion concentration gradient increase with peak-to-peak potential and decrease with frequency.

$\mathrm{V}_{\mathrm{pp}}$ dependency results showed that intensity increase thus ion concentration was generally increasing with peak-to-peak potential. Ion 
enrichment was negligible at $5 \mathrm{~V}$ and started to increase as the potential increased from $5 \mathrm{~V}$ to $10 \mathrm{~V}$.

Such increase with $\mathrm{V}_{\mathrm{pp}}$ can be understood from the aspect of ion migration. Higher potential provides stronger electric field thus ion migrates further at each half cycle resulting in a greater biased movement from two half cycles with the magnitude increase in proportional with the applied potential.

Frequency dependency results showed that ion enrichment effect decreased with frequency. However, the ion enrichment is still noticeable even at $400 \mathrm{~Hz}$ which is 11 times higher than the electrode charging frequency.

Similarly, from the aspect on the magnitude of ion migration, such decrease with frequency has also been reported by previous theoretical studies. Golovnev et al. [168] reported an analytical solution of the PNP equation under applied AC potentials and concluded that the change of ion concentration with time is inversely related to angular velocity $(\omega=2 \pi f)$ of the applied signal thus also inversely related to the applied frequency, which matches our experimental results. This is attributed to the decrease of time available for momentum transfer at each half cycle between ion and signal. The increase of frequency will decrease the time that the averaged force in each half cycle applied on the ions thus decrease the magnitude of ion momentum increase and decrease the migration velocity and rate.

Also, converting such relative frequency to the normally used solution gives: $\sim 6 \mathrm{kHz}$ for $1 \mathrm{mS} / \mathrm{m} \mathrm{KCl}$ solution used for $50 \mathrm{kHz}$ for ACEO 
experiments; $\sim 50 \mathrm{kHz}$ for $0.1 \mathrm{~S} / \mathrm{m} \mathrm{NaCl}$ solution used for DEP experiments and $>500 \mathrm{kHz}$ for $0.9 \mathrm{NaCl}$ solution which providing equal osmolarity in human body. As a result, such ion concentration gradient existed in a fairly wide range and should not be neglected.

\subsection{Conclusions}

This work spatially and temporally examined the formation of ion concentration gradient in spatially non-uniform AC electric fields via spatial detection of FITC ion emission intensity.

Ion enrichment and concentration gradient were observed in higher electric field density areas over the course of $60 \mathrm{~s}$ experiment. Despite of the fact that applied AC potential oscillating symmetrically at a high relative frequency, biased FITC ion electromigration was observed, induced great ion enrichment up to 4 times of initial ion concentration.

Further, spatial variances in the ion migration thus the ion concentration gradient establishment were observed in contour plots. Intensity [FITC] depletion was observed in lower electric field density areas and [FITC] enrichment was observed in higher electric field density areas while the overall averaged intensity across the whole inspected area stayed almost unchanged due to the mass conservation.

Lastly, potential and frequency dependencies were examined. Biased electromigration magnitude thus ion enrichment magnitude increases were 
observed with potential increases due to the increase of strength of electric field thus higher magnitude of electromigration during each half oscillation periods. Biased electromigration magnitude decreases were observed with frequency increases due to the decrease of time available at each cycle thus lower the magnitude of ion momentum increase.

This work demonstrates that biased ion migration existed in spatially non-uniform $\mathrm{AC}$ electric field at high relative frequencies resulting in ion enrichment and ion concentration gradient establishment. Such non-uniformities in solution properties may induce anomalous (bio)particle dielectrophoretic responses and hydrodynamic fluid behaviors even causing solution biocompatibilities issues. The relative frequency and $\mathrm{V}_{\mathrm{pp}}$ range inspected in this work may facilitate the design of operational condition in microfluidics experiments to avoid such media condition change. 


\section{Chapter 6. CONCLUSIONS AND FUTURE WORKS}

\subsection{Introduction}

Micro- and nanofluidic devices have been developed toward miniaturizing multiple analytical and diagnostic techniques for portable, low cost and easy accessible applications. Device fluidic channel dimensions have been developing from centimeter, millimeter scale to micrometer even nanometer scales. Such micro and nanometer scale channels then increases the feasibility for fluidic system integration. Such integrated fluidic system in combination with the well developed micro- and nano- electrodes techniques would make the targeted portable techniques becomes more realizable. AC electrokinetics are highly favorable in micro and nanofluidic devices since they can exert forces or transfer momentums to (bio)particles, charged molecules (DNAs) and (bio)fluids by signals generated from the small scale electrodes. It has been shown both theoretically and experimentally that AC electrokinetics are highly dependent on electrolytic fluidic conditions and such important term have been previously assumed to be constant in most of published works. The work described in this dissertation has been initiated by an encountered non-ideal cell deformation in an AC DEP device under the impact of electric fields. Such cell deformation potentially affects cell property and viability thus changes cell response to electric field and other behaviors. Multiple possible physical mechanisms is examined on such cell deformation in the first part of this dissertation and is concluded to be induced by solution biocompatibility change in microdevices driven by AC electric fields. General electrolyte electrochemical reactions represented by $\mathrm{pH}$ change and mass transport represented by ion concentration gradient are then studied to 
understand and propose solution to such solution biocompatibility change problem. It was found that both solution $\mathrm{pH}$ and ion concentration have been altered significantly from initial state and strong spatial $\mathrm{pH}$ gradient and spatial ion concentration gradient have been established.

\subsection{Biocompatibility Change in Spatially Non-uniform AC DEP Field}

The work performed in Chapter 3 described the human red blood cell crenation behavior. Through multiple control experiments inspecting effects from temperature, induced flow, DEP forces, biocompatibility and electroporation, it has been proved that such cell crenation was induced by the change of fluid biocompatibility. Further, the study showed that such biocompatibility could be induced by the formation of local ion concentration gradient or the combination of ion concentration and $\mathrm{pH}$ gradient in such $\mathrm{AC}$ DEP system. Such change in $\mathrm{pH}$ and ion concentration would affect the biocompatibility from multiple aspects such as solution acidity, osmolarity and ionic strength. Those solution properties changes would further affect cell membrane structure, water and ion fluxes and other intra and extra cellular properties.

Chapter 3 proved the existence of $\mathrm{pH}$ and ion concentration change and spatial gradients in non-uniform AC electric fields by the observation of red blood cell crenation. Such observation can be a very good example for the non-constant value of solution properties under AC electric fields and how they can affect (bio)particles in the system. 
However, since red blood cells crenation behaviors are affected by numerous other factors such as temperature, electric fields even flow, they cannot be perfect indicators for either $\mathrm{pH}$ value or ion concentration. Also, the contribution from $\mathrm{pH}$ and ion concentration on cell crenation should be further separated and detected individually, which have been completed in Chapter 4 and 5 .

\subsection{Detection of $\mathrm{pH}$ Change and $\mathrm{pH}$ Spatial Gradient in Spatially Non-uniform AC DEP Field}

The work performed in Chapter 4 described the $\mathrm{pH}$ change in microfluidics under spatially non-uniform AC electric field detected by Fluorescein sodium salt. The frequency range was chosen to be at and above the theoretical electrode charging frequency in order to explore the 'safe' frequency range where no $\mathrm{pH}$ change thus Faradaic reaction can be induced by the applied electrical potential. Spatial, time, applied potential and frequency dependencies were also examined to better understand the formation process and distribution of $\mathrm{pH}$ concentration gradient and the dependence on input signals.

From results in Chapter 4, the observed 2.5 units change of $\mathrm{pH}$ proved the existence of Faradaic reaction existed in the entire $6.3 \times 10^{5} \mu \mathrm{m}^{2}$ chamber in the examined microfluidic system over the $120 \mathrm{~s}$ experiment. It was also observed that the $\mathrm{pH}$ pattern follows to the similar trend with first order reaction, which means the reaction rate is high at the very beginning and decrease with time increase. Spatially, strong $\mathrm{pH}$ gradient at $2 \times 10^{4} \mathrm{unit} / \mu \mathrm{m}$ was observed which created a spatially non-uniform solution environment in the entire fluidic chamber. For the last, Faradaic reaction rate increases were 
observed with increasing potential and Faradaic reaction rate decreases were observed with increasing frequency till relative frequency equals to 3.5 where negligible $\mathrm{pH}$ change or Faradaic reaction was observed.

The work presented in Chapter 4 is, by our best knowledge, the first work examining the spatially non-uniform $\mathrm{pH}$ gradient distribution in $\mathrm{AC}$ microfluidics system. The significance of impact from Faradaic reaction on solution property change and non-uniformity have been examined. Frequency and potential ranges for possible Faradaic reaction have been defined. These $\mathrm{pH}$ changes may induce non-ideal (bio)particle DEP responses and affecting the performance of ACEO pumps or related hydrodynamic phenomena.

Potential future work still exists on this topic:

- The work in Chapter 4 only detected $\mathrm{pH}$ change in $4 \mathrm{mM} \mathrm{NaCl}$ solution. Other electrolytes have also been applied in microfluidics system at various concentrations. $\mathrm{KCl}$ has been applied at different concentrations in ACEO and ACET experiments due to its strong ion symmetricity. PBS solution at different conductivities with polysaccharide have been used for bio-particle DEP experiments for its strong biocompatibility. $\mathrm{pH}$ changes in those electrolytes at different concentrations should be inspected.

- The work in Chapter 4 only detected $\mathrm{pH}$ change in orthogonally located electrodes. Other electrodes designs such as quadruple electrodes, interdigitated electrodes and parallel electrode array have been widely applied in different electrokinetic microfluidic systems. 
Each system has its own characteristic lengths and electric field shapes. Although the characteristic length have been considered in the relative frequency equation, future work should be completed on each electrode design to map the spatial $\mathrm{pH}$ gradients induced by varied electric field shapes.

- The work in Chapter has detected $\mathrm{pH}$ using $\mathrm{pH}$ sensitive ion indicators. $\mathrm{pH}$ values were obtained from relationship between emission intensity and $\mathrm{pH}$ values. Such technique is limited by the microscope camera recording fps speed and exposure time. Since the experiments have been processed under $\mathrm{kHz}$ range, it would be almost impossible to perform period-to-period analysis by this technique. Future measurements should be performed use electronic devices such as oscilloscope, ampere meter, voltmeter or electrochemical impedance spectroscopy to measure the amplitude and phase of current and real potential applied on the microdevice. More insight on Faradaic current could be obtained using such techniques.

\subsection{Detection of Ion Concentration Change and Ion Concentration Spatial Gradient in Spatially Non-uniform AC DEP Field}

The work performed in Chapter 5 described the ion concentration change and spatial ion concentration variation induced by only non-Faradaic process in microfluidic systems under AC electric fields. Impacts from Faradaic processes have been carefully controlled by depositing of dielectric layer on electrode and using methanol as inert organic solvent. Fluorescing ionized FITC molecule has been used to represent ion concentration in the system. Similarly, frequency range was chosen to be at and above the theoretical 
electrode charging frequency in order to protect electrode and also explore the 'safe' frequency range where no ion concentration gradient would be induced by the non-Faradaic process.

From results in Chapter 5, ion concentration gradient was observed with the highest magnitude of 4 times of initial concentration over the course of 60 $\mathrm{s}$ experimental time under the oscillating AC electric field. Spatial variance of ion concentration gradient was also observed and was related to electric field density. Ion enrichment was observed at higher field density areas while ion depletion was observed at lower field density areas. The total ion concentration across the whole microfluidic chamber was generally stayed at constant due to mass balance except minor effects from remained Faradaic process induced $\mathrm{pH}$ change and electrolyte diffusion from connection channels designed for connecting chamber to inlet and outlet in the microfluidic system. Results from potential and frequency dependencies showed that magnitude of ion concentration gradient increased with potential and decreased with frequency. Frequencies higher than 11 times of electrode charging frequency was defined as 'safe' frequency region since negligible ion concentration gradient have been observed and the solution ion concentration was remained constant and uniform through the entire experiments.

The work in Chapter 4 isolated non-Faradaic process from Faradaic process and inspected the effect of ion migration and diffusion in microfluidic systems under the impact of spatially non-uniform AC electric field. It has been proved by control experiments that such ion concentration gradient was induced by the spatially varied electric field density provided by non-uniform 
electric field. Due to the requirements of non-uniform electric field by most of AC electrokinetic applications such as DEP ACEO and ACET, such ion concentration gradient could exist in numerous microfluidics systems inducing non-ideal effects such as change of conductivity, electrical permittivity, ionic strength, temperature change induced by Joule heating and solution biocompatibility.

However, potential future works still exist on this topic:

- The work demonstrated in Chapter 5 used a microdevice coated by dielectric layer on the entire glass slide. Such layer might then alter the shape and strength of electric field and also the potential drop across the real solution part. As a result, another mask should be made in the future to coat the dielectric material only on top of electrode to minimize the impact from the dielectric layer but still isolate the Faradaic process from non-Faradaic process

- The work demonstrated in Chapter 5 showed minor Faradaic process. Although the magnitude of Faradaic process is low, it still affected the detection accuracy of the ion concentration gradient from non-Faradaic process, especially at long time ranges since the non-Faradaic process has a much shorter time scale than the Faradaic process. Such issue could be solved by optimization of the dielectric coating process or electrode deposition process to decrease electrode thickness and increase dielectric layer thickness to further minimize the contact between electrode and electrolyte solution. Also, more inert organic 
solvents than methanol such as DMSO could be applied as solvent, although the current issue is DMSO is corruptive to the PDMS fluidic layer.

- The work presented in Chapter 5 inspected the dependencies of ion concentration on time, potential and frequency. It has also been reported, although in plasma research area rather than electrochemistry systems where the ions were studied in vacuum condition thus effects of diffusion is much lesser, that the initial phase of applied signal can also affect the ion oscillation trajectories, even under AC electric field. Such dependency would be also very interesting to inspect in the microfluidic systems.

- The frequency dependency was inspected in Chapter 5 were at $\mathrm{kHz}$ range, which would not allow us to visually see the biased migration or directional oscillation due to the limitation of microscope camera fps. Attempts have been made on discovering the ion oscillations under lower frequencies (i.e., 0.5 or $1 \mathrm{~Hz}$ ). However, the minor Faradaic reaction at those low frequencies becomes significant and greatly affected the data quality. As a result, detection of ion concentration oscillation under lower frequencies would be favorable once the Faradaic process issue is solved.

- Other solvent with different viscosity thus diffusivity should be applied to further study the diffusion behavior. 
- Similar to the descriptions in section 6.3 , the inspection on other electrolyte, electrode designs and performing measurements using electronic devices would be also beneficial to this non-Faradaic process. Especially for the electrical measurements, non-Faradaic processes including electromigration and diffusion should be measured before performing the Faradaic measurements in order to be able to well recognize the contribution of output current from each process.

\subsection{Contribution to the Discipline}

The works presented in this dissertation started from an observation of failure in AC DEP microfluidic system. Multiple physical mechanisms have been inspected and it has been proved that the device failure was induced by solution biocompatibility change according to the results from carefully designed control experiments.

Faradaic and non-Faradaic processes were then inspected to reveal the mechanism and magnitude of solution property change induced by the electrochemical processes under spatially non-uniform AC electric fields. It has been proved that both $\mathrm{pH}$ and ion concentration changed significantly with time despite the oscillation nature of $\mathrm{AC}$ electric fields. Strong $\mathrm{pH}$ and ion concentration spatial gradient have also been found due to the non-uniformity of AC electric fields. Potential and frequency dependency have also been inspected. Both $\mathrm{pH}$ and ion concentration change increased with increase of applied potential and decreased with increase of applied frequency. Negligible $\mathrm{pH}$ change has been found at frequencies above 3.5 times of electrode 
charging frequency while ion concentration change existed at frequencies up to 11 times of electrode charging frequency.

This work demonstrated the significance of solution property change under the impact of externally applied AC electric potential. Such solution property change under $\mathrm{AC}$ electric field induced by electric field spatial non-uniformity thus should be considered when performing system design and data analysis to obtain better predictable and accurate data. Also, operational conditions should be designed to avoid the frequency ranges that non-ideal solution property change would occur described in this dissertation. 


\section{REFERENCES}

1. Nguyen, N.T., X.Y. Huang, and T.K. Chuan, MEMS-micropumps: A review. Journal of Fluids Engineering-Transactions of the Asme, 2002. 124(2): p. 384-392.

2. Judy, J.W., Microelectromechanical systems (MEMS): fabrication, design and applications. Smart Materials \& Structures, 2001. 10(6): p. 1115-1134.

3. Madou, M.J., Fundamentals of Microfabrication: The Science of Miniaturization, Second Edition. 2002: Taylor \& Francis.

4. Kim, P., K.W. Kwon, M.C. Park, S.H. Lee, S.M. Kim, and K.Y. Suh, Soft lithography for microfluidics: a review. Biochip Journal, 2008. 2(1): p. 1-11.

5. Petersen, K.E., SILICON AS A MECHANICAL MATERIAL. Proceedings of the leee, 1982. 70(5): p. 420-457.

6. Squires, T.M. and S.R. Quake, Microfluidics: Fluid physics at the nanoliter scale. Reviews of Modern Physics, 2005. 77(3): p. 977-1026.

7. Persat, A., R.D. Chambers, and J.G. Santiago, Basic principles of electrolyte chemistry for microfluidic electrokinetics. Part I: Acid-base equilibria and pH buffers. Lab on a Chip, 2009. 9(17): p. 2437-2453.

8. Persat, A., M.E. Suss, and J.G. Santiago, Basic principles of electrolyte chemistry for microfluidic electrokinetics. Part II: Coupling between ion mobility, electrolysis, and acid-base equilibria. Lab on a Chip, 2009. 9(17): p. 2454-2469.

9. Verpoorte, E. and N.F. De Rooij, Microfluidics meets MEMS. Proceedings of the leee, 2003. 91(6): p. 930-953.

10. Whitesides, G.M., The origins and the future of microfluidics. Nature, 2006. 442(7101): p. 368-373.

11. Fan, L.S., Y.C. Tai, and R.S. Muller, IC-PROCESSED ELECTROSTATIC MICROMOTORS. Sensors and Actuators, 1989. 20(1-2): p. 41-47.

12. Terry, S.C., J.H. Jerman, and J.B. Angell, GAS-CHROMATOGRAPHIC AIR ANALYZER FABRICATED ON A SILICON-WAFER. leee Transactions on Electron Devices, 1979. 26(12): p. 1880-1886.

13. Seiler, K., D.J. Harrison, and A. Manz, PLANAR GLASS CHIPS FOR CAPILLARY ELECTROPHORESIS - REPETITIVE SAMPLE INJECTION, QUANTITATION, AND SEPARATION EFFICIENCY. Analytical Chemistry, 1993. 65(10): p. 1481-1488.

14. SalimiMoosavi, H., T. Tang, and D.J. Harrison, Electroosmotic pumping of organic solvents and reagents in microfabricated reactor chips. Journal of the American Chemical Society, 1997. 119(37): p. 8716-8717.

15. Ermakov, S.V., S.C. Jacobson, and J.M. Ramsey, Computer simulations of electrokinetic transport in microfabricated channel structures. Analytical Chemistry, 1998. 70(21): p. 4494-4504.

16. Woolley, A.T. and R.A. Mathies, ULTRA-HIGH-SPEED DNA-SEQUENCING USING CAPILLARY ELECTROPHORESIS CHIPS. Analytical Chemistry, 1995. 67(20): p. 3676-3680. 
17. Ng, J.M.K., I. Gitlin, A.D. Stroock, and G.M. Whitesides, Components for integrated poly(dimethylsiloxane) microfluidic systems. Electrophoresis, 2002. 23(20): p. 3461-3473.

18. Sia, S.K. and G.M. Whitesides, Microfluidic devices fabricated in poly(dimethylsiloxane) for biological studies. Electrophoresis, 2003. 24(21): p. 3563-3576.

19. Beebe, D.J., G.A. Mensing, and G.M. Walker, Physics and applications of microfluidics in biology. Annual Review of Biomedical Engineering, 2002. 4: p. 261-286.

20. McDonald, J.C., D.C. Duffy, J.R. Anderson, D.T. Chiu, H.K. Wu, O.J.A. Schueller, and G.M. Whitesides, Fabrication of microfluidic systems in poly(dimethylsiloxane). Electrophoresis, 2000. 21(1): p. 27-40.

21. Lyklema, J., Fundamentals of Interface and Colloid Science: Particulate Colloids. 2005: Elsevier Science.

22. Ramos, A., H. Morgan, N.G. Green, and A. Castellanos, Ac electrokinetics: a review of forces in microelectrode structures. Journal of Physics D-Applied Physics, 1998. 31(18): p. 2338-2353.

23. Morgan, H. and N.G. Green, AC Electrokinetics: Colloids and Nanoparticles. 2003: Research Studies Press.

24. Chang, C.C. and R.J. Yang, Electrokinetic mixing in microfluidic systems. Microfluidics and Nanofluidics, 2007. 3(5): p. 501-525.

25. Jones, T.B. and T.B. Jones, Electromechanics of Particles. 2005: Cambridge University Press.

26. Srivastava, S.K., J.I. Baylon-Cardiel, B.H. Lapizco-Encinas, and A.R. Minerick, A continuous DC-insulator dielectrophoretic sorter of microparticles. Journal of Chromatography A, 2011. 1218(13): p. 1780-1789.

27. Srivastava, S.K., A. Gencoglu, and A.R. Minerick, DC insulator dielectrophoretic applications in microdevice technology: a review. Analytical and Bioanalytical Chemistry, 2011. 399(1): p. 301-321.

28. Srivastava, S.K., A. Artemiou, and A.R. Minerick, Direct current insulator-based dielectrophoretic characterization of erythrocytes: ABO-Rh human blood typing. Electrophoresis, 2011. 32(18): p. 2530-2540.

29. Minerick, A.R., A.E. Ostafin, and H.C. Chang, Electrokinetic transport of red blood cells in microcapillaries. Electrophoresis, 2002. 23(14): p. 2165-2173.

30. Leonard, K.M. and A.R. Minerick, Explorations of ABO-Rh antigen expressions on erythrocyte dielectrophoresis: Changes in cross-over frequency. Electrophoresis, 2011. 32(18): p. 2512-2522.

31. Minerick, A.R., R.H. Zhou, P. Takhistov, and H.C. Chang, Manipulation and characterization of red blood cells with alternating current fields in microdevices. Electrophoresis, 2003. 24(21): p. 3703-3717.

32. Minerick, A.R., The rapidly growing field of micro and nanotechnology to measure living cells. Aiche Journal, 2008. 54(9): p. 2230-2237.

33. Gupta, V., I. Jafferji, M. Garza, V.O. Melnikova, D.K. Hasegawa, R. Pethig, and D.W. Davis, 
ApoStream (TM), a new dielectrophoretic device for antibody independent isolation and recovery of viable cancer cells from blood. Biomicrofluidics, 2012. 6(2).

34. Davis, D., C. Neal, J. Woo, V. Melnikova, K. Anderes, E. Somers, and D. O'Shannessy, Antibody-Independent ApoStream (TM) Technology Isolates Folate Receptor Alpha Positive Circulating Tumor Cells from Blood of Non-Small Cell Lung Cancer Patients. Journal of Thoracic Oncology, 2012. 7(9): p. S284-S284.

35. Adams, T.N.G., K.M. Leonard, and A.R. Minerick, Frequency sweep rate dependence on the dielectrophoretic response of polystyrene beads and red blood cells. Biomicrofluidics, 2013. 7(6).

36. Ramos, A., H. Morgan, N.G. Green, and A. Castellanos, AC electric-field-induced fluid flow in microelectrodes. Journal of Colloid and Interface Science, 1999. 217(2): p. 420-422.

37. Green, N.G., A. Ramos, A. Gonzalez, H. Morgan, and A. Castellanos, Fluid flow induced by nonuniform ac electric fields in electrolytes on microelectrodes. I. Experimental measurements. Physical Review E, 2000. 61(4): p. 4011-4018.

38. Gonzalez, A., A. Ramos, N.G. Green, A. Castellanos, and H. Morgan, Fluid flow induced by nonuniform ac electric fields in electrolytes on microelectrodes. II. A linear double-layer analysis. Physical Review E, 2000. 61(4): p. 4019-4028.

39. Green, N.G., A. Ramos, A. Gonzalez, H. Morgan, and A. Castellanos, Fluid flow induced by nonuniform ac electric fields in electrolytes on microelectrodes. III. Observation of streamlines and numerical simulation. Physical Review E, 2002. 66(2).

40. Ramos, A., A. Gonzalez, A. Castellanos, N.G. Green, and H. Morgan, Pumping of liquids with ac voltages applied to asymmetric pairs of microelectrodes. Physical Review E, 2003. 67(5).

41. Ajdari, A., Pumping liquids using asymmetric electrode arrays. Physical Review E, 2000. 61(1): p. R45-R48.

42. Wu, J., Y.X. Ben, and H.C. Chang, Particle detection by electrical impedance spectroscopy with asymmetric-polarization AC electroosmotic trapping. Microfluidics and Nanofluidics, 2005. 1(2): p. 161-167.

43. Wu, J., ac electro-osmotic micropump by asymmetric electrode polarization. Journal of Applied Physics, 2008. 103(2).

44. Ramos, A., H. Morgan, N.G. Green, A. Gonzalez, and A. Castellanos, Pumping of liquids with traveling-wave electroosmosis. Journal of Applied Physics, 2005. 97(8).

45. Khair, A.S. and T.M. Squires, Fundamental aspects of concentration polarization arising from nonuniform electrokinetic transport (vol 20, art no 087102, 2008). Physics of Fluids, 2008. 20(12).

46. Lian, M. and J. Wu, Microfluidic flow reversal at low frequency by AC electrothermal effect. Microfluidics and Nanofluidics, 2009. 7(6): p. 757-765.

47. Hong, F.J., J. Cao, and P. Cheng, A parametric study of AC electrothermal flow in microchannels with asymmetrical interdigitated electrodes. International Communications in Heat and Mass Transfer, 2011. 38(3): p. 275-279. 
48. Du, E. and S. Manoochehri, Enhanced ac electrothermal fluidic pumping in microgrooved channels. Journal of Applied Physics, 2008. 104(6).

49. Sotira, Y., Y. Kun?Lin, and T. Costas, Electrical Double?Layer Formation, in Dekker Encyclopedia of Nanoscience and Nanotechnology. 2007, Taylor \& Francis. p. 1001-1014.

50. Yang, K.-L., S. Yiacoumi, and C. Tsouris, Electrical Double-Layer Formation. Dekker Encyclopedia of Nanoscience and Nanotechnology, 2004: p. 1001.

51. Bard, F., Electrochemical methods Fundamentals and Applications Second Edition. 2000.

52. Newman, J. and K.E. Thomas-Alyea, Electrochemical Systems. 2004: John Wiley \& Sons.

53. Grahame, D.C., Electrode Processes And the Electrical Double Layer. Annual Review of Physical Chemistry, 1956. 6: p. 337-358.

54. Eyring, H., S. Glasstone, and K.J. Laidler, Application of the Theory of Absolute Reaction Rates to Overvoltage. The Journal of Chemical Physics, 1939. 7(11): p. 1053-1065.

55. Kimball, G.E., The Absolute Rates of Heterogeneous Reactions II. Electrode Reactions. The Journal of Chemical Physics, 1940. 8(2): p. 199-204.

56. Kimball, G.E., S. Glasstone, and A. Glassner, Overvoltage and the Structure of the Electrical Double Layer at a Hydrogen Electrode. The Journal of Chemical Physics, 1941. 9(1): p. 91-96.

57. Kirby, B.J. and E.F. Hasselbrink, Zeta potential of microfluidic substrates: 1. Theory, experimental techniques, and effects on separations. Electrophoresis, 2004. 25(2): p. 187-202.

58. Kirby, B.J. and E.F. Hasselbrink, Zeta potential of microfluidic substrates: 2. Data for polymers. Electrophoresis, 2004. 25(2): p. 203-213.

59. Garcia-Sanchez, P., A. Ramos, N.G. Green, and H. Morgan, Traveling-Wave Electrokinetic Micropumps: Velocity, Electrical Current, and Impedance Measurements. Langmuir, 2008. 24(17): p. 9361-9369.

60. Bard, A.J., Electroanalytical Chemistry: A Series of Advances. 1988: Taylor \& Francis.

61. Hayden, J.L.R., Alternating-Current Electrolysis. Conference: Meeting of the American Institute of Electrical Engineers, New York, 1907: p. 201.

62. Allmand, A.J. and H.C. Cocks, CCCXLVII.-The electrolysis of potassium chloride solutions by alternating currents. Journal of the Chemical Society (Resumed), 1927(0): p. 2626-2639.

63. Shipley, J.W., THE ALTERNATING CURRENT ELECTROLYSIS OF WATER. Canadian Journal of Research, 1929. 1(4): p. 305-358.

64. Kreuer, K.D., S.J. Paddison, E. Spohr, and M. Schuster, Transport in proton conductors for fuel-cell applications: Simulations, elementary reactions, and phenomenology. Chemical Reviews, 2004. 104(10): p. 4637-4678.

65. Zawodzinski, T.A., C. Derouin, S. Radzinski, R.J. Sherman, V.T. Smith, T.E. Springer, and S. Gottesfeld, WATER-UPTAKE BY AND TRANSPORT THROUGH NAFION(R) 117 MEMBRANES. Journal of the Electrochemical Society, 1993. 140(4): p. 1041-1047.

66. Read, J., K. Mutolo, M. Ervin, W. Behl, J. Wolfenstine, A. Driedger, and D. Foster, Oxygen transport properties of organic electrolytes and performance of lithium/oxygen battery. 
Journal of the Electrochemical Society, 2003. 150(10): p. A1351-A1356.

67. Islam, M.S., D.J. Driscoll, C.A.J. Fisher, and P.R. Slater, Atomic-scale investigation of defects, dopants, and lithium transport in the LiFePO4 olivine-type battery material. Chemistry of Materials, 2005. 17(20): p. 5085-5092.

68. Amatore, C., B. Fosset, J. Bartelt, M.R. Deakin, and R.M. Wightman, ELECTROCHEMICAL KINETICS AT MICROELECTRODES .5. MIGRATIONAL EFFECTS ON STEADY OR QUASI-STEADY-STATE VOLTAMMOGRAMS. Journal of Electroanalytical Chemistry, 1988. 256(2): p. 255-268.

69. Ciszkowska, M. and J.G. Osteryoung, VOLTAMMETRY OF METALS AT MERCURY FILM MICROELECTRODES IN THE ABSENCE AND THE PRESENCE OF VARYING CONCENTRATIONS OF SUPPORTING ELECTROLYTE. Analytical Chemistry, 1995. 67(6): p. 1125-1131.

70. Horrocks, B.R., M.V. Mirkin, D.T. Pierce, A.J. Bard, G. Nagy, and K. Toth, SCANNING ELECTROCHEMICAL MICROSCOPY .19. ION-SELECTIVE POTENTIOMETRIC MICROSCOPY. Analytical Chemistry, 1993. 65(9): p. 1213-1224.

71. Morris, R.B., K.F. Fischer, and H.S. White, ELECTROCHEMISTRY OF ORGANIC REDOX LIQUIDS REDUCTION OF 4-CYANOPYRIDINE. Journal of Physical Chemistry, 1988. 92(18): p. 5306-5313.

72. Moya, A.A., J. Castilla, and J. Horno, IONIC TRANSPORT IN ELECTROCHEMICAL-CELLS INCLUDING ELECTRICAL DOUBLE-LAYER EFFECTS - A NETWORK THERMODYNAMICS APPROACH. Journal of Physical Chemistry, 1995. 99(4): p. 1292-1298.

73. Myland, J.C. and K.B. Oldham, GENERAL-THEORY OF STEADY-STATE VOLTAMMETRY. Journal of Electroanalytical Chemistry, 1993. 347(1-2): p. 49-91.

74. Paulson, S.C., N.D. Okerlund, and H.S. White, Diffusion currents in concentrated redox solutions. Analytical Chemistry, 1996. 68(4): p. 581-584.

75. Smith, C.P. and H.S. White, THEORY OF THE VOLTAMMETRIC RESPONSE OF ELECTRODES OF SUBMICRON DIMENSIONS - VIOLATION OF ELECTRONEUTRALITY IN THE PRESENCE OF EXCESS SUPPORTING ELECTROLYTE. Analytical Chemistry, 1993. 65(23): p. 3343-3353.

76. Wei, C., A.J. Bard, G. Nagy, and K. Toth, SCANNING ELECTROCHEMICAL MICROSCOPY .28. ION-SELECTIVE NEUTRAL CARRIER-BASED MICROELECTRODE POTENTIOMETRY. Analytical Chemistry, 1995. 67(8): p. 1346-1356.

77. Shih, S.C.C., I. Barbulovic-Nad, X.N. Yang, R. Fobel, and A.R. Wheeler, Digital microfluidics with impedance sensing for integrated cell culture and analysis. Biosensors \& Bioelectronics, 2013. 42: p. 314-320.

78. Pradhan, R., L. Das, J. Chatterjee, M.M.A. Mitra, and S. Das, Monopolar Impedance Sensing Microdevices for Characterization of Cells and Tissue Culture. Sensor Letters, 2013. 11(3): p. 466-475.

79. Sarro, E., M. Lecina, A. Fontova, C. Sola, F. Godia, J.J. Cairo, and R. Bragos, Electrical impedance spectroscopy measurements using a four-electrode configuration improve on-line monitoring of cell concentration in adherent animal cell cultures. Biosensors \& 
Bioelectronics, 2012. 31(1): p. 257-263.

80. Hong, J., D.S. Yoon, S.K. Kim, T.S. Kim, S. Kim, E.Y. Pak, and K. No, AC frequency characteristics of coplanar impedance sensors as design parameters. Lab on a Chip, 2005. 5(3): p. 270-279.

81. Timmer, B., W. Sparreboom, W. Olthuis, P. Bergveld, and A. van den Berg, Optimization of an electrolyte conductivity detector for measuring low ion concentrations. Lab on a Chip, 2002. 2(2): p. 121-124.

82. Singh, G. and R.F. Saraf, Direct Measurement of Ion Accumulation at the Electrode Electrolyte Interface under an Oscillatory Electric Field. The Journal of Physical Chemistry B, 2006. 110(25): p. 12581-12587.

83. Bazant, M.Z., M.S. Kilic, B.D. Storey, and A. Ajdari, Nonlinear electrokinetics at large voltages. New Journal of Physics, 2009. 11.

84. Bazant, M.Z., K. Thornton, and A. Ajdari, Diffuse-charge dynamics in electrochemical systems. Physical Review E, 2004. 70(2).

85. Olesen, L.H., M.Z. Bazant, and H. Bruus, Strongly nonlinear dynamics of electrolytes in large ac voltages. Physical Review E, 2010. 82(1).

86. Rubinstein, I. and B. Zaltzman, Dynamics of extended space charge in concentration polarization. Physical Review E, 2010. 81(6).

87. Rubinstein, I. and B. Zaltzman, Extended space charge in concentration polarization. Advances in Colloid and Interface Science, 2010. 159(2): p. 117-129.

88. Chu, K.T. and M.Z. Bazant, Nonlinear electrochemical relaxation around conductors. Physical Review E, 2006. 74(1).

89. Kilic, M.S., M.Z. Bazant, and A. Ajdari, Steric effects in the dynamics of electrolytes at large applied voltages. I. Double-layer charging. Physical Review E, 2007. 75(2).

90. Kilic, M.S., M.Z. Bazant, and A. Ajdari, Steric effects in the dynamics of electrolytes at large applied voltages. II. Modified Poisson-Nernst-Planck equations. Physical Review E, 2007. 75(2).

91. Suh, Y.K. and S. Kang, Asymptotic analysis of ion transport in a nonlinear regime around polarized electrodes under ac. Physical Review E, 2008. 77(3).

92. Suh, Y.K. and S. Kang, Asymptotic analysis of ion transport in a nonlinear regime around polarized electrodes under ac. Physical Review E, 2008. 77(3): p. 031504.

93. Zhang, W., Chen, Frequency effects on periodical potential and ion concentration oscillation in microchannel with and AC electric field perpendicular to wall. Applied Mathematical Sciences, 2011. 5(33): p. 1631 - 1647.

94. Bazant, M.Z. and T.M. Squires, Induced-charge electrokinetic phenomena: Theory and microfluidic applications. Physical Review Letters, 2004. 92(6).

95. Yossifon, G. and H.C. Chang, Selection of Nonequilibrium Overlimiting Currents: Universal Depletion Layer Formation Dynamics and Vortex Instability. Physical Review Letters, 2008. 101(25).

96. Cheng, L.-J. and L.J. Guo, Rectified Ion Transport through Concentration Gradient in 
Homogeneous Silica Nanochannels. Nano Letters, 2007. 7(10): p. 3165-3171.

97. Daiguji, H., Ion transport in nanofluidic channels. Chemical Society Reviews, 2010. 39(3): p. 901-911.

98. Höltzel, A. and U. Tallarek, lonic conductance of nanopores in microscale analysis systems: Where microfluidics meets nanofluidics. Journal of Separation Science, 2007. 30(10): p. 1398-1419.

99. Karnik, R., R. Fan, M. Yue, D. Li, P. Yang, and A. Majumdar, Electrostatic Control of lons and Molecules in Nanofluidic Transistors. Nano Letters, 2005. 5(5): p. 943-948.

100. Kjeang, E., N. Djilali, and D. Sinton, Microfluidic fuel cells: A review. Journal of Power Sources, 2009. 186(2): p. 353-369.

101. Li, W., L. Ding, Q. Wang, and B. Su, Differential pulse voltammetry detection of dopamine and ascorbic acid by permselective silica mesochannels vertically attached to the electrode surface. Analyst, 2014. 139(16): p. 3926-3931.

102. Nandigana, V.V.R. and N.R. Aluru, Nonlinear Electrokinetic Transport Under Combined ac and dc Fields in Micro/Nanofluidic Interface Devices. Journal of Fluids Engineering-Transactions of the Asme, 2013. 135(2).

103. Napoli, M., J.C.T. Eijkel, and S. Pennathur, Nanofluidic technology for biomolecule applications: a critical review. Lab on a Chip, 2010. 10(8): p. 957-985.

104. Plecis, A., R.B. Schoch, and P. Renaud, Ionic Transport Phenomena in Nanofluidics: Experimental and Theoretical Study of the Exclusion-Enrichment Effect on a Chip. Nano Letters, 2005. 5(6): p. 1147-1155.

105. Tang, G., D. Yan, C. Yang, H. Gong, J.C. Chai, and Y.C. Lam, Assessment of Joule heating and its effects on electroosmotic flow and electrophoretic transport of solutes in microfluidic channels. ELECTROPHORESIS, 2006. 27(3): p. 628-639.

106. van der Heyden, F.H.J., D.J. Bonthuis, D. Stein, C. Meyer, and C. Dekker, Power Generation by Pressure-Driven Transport of Ions in Nanofluidic Channels. Nano Letters, 2007. 7(4): p. 1022-1025.

107. Zangle, T.A., A. Mani, and J.G. Santiago, Theory and experiments of concentration polarization and ion focusing at microchannel and nanochannel interfaces. Chemical Society Reviews, 2010. 39(3): p. 1014-1035.

108. An, R., D.O. Wipf, and A.R. Minerick, Spatially variant red blood cell crenation in alternating current non-uniform fields. Biomicrofluidics, 2014. 8(2).

109. Arya, S.K., K.C. Lee, D. Bin Dah'alan, Daniel, and A.R.A. Rahman, Breast tumor cell detection at single cell resolution using an electrochemical impedance technique. Lab on a Chip, 2012. 12(13): p. 2362-2368.

110. Lapizco-Encinas, B.H., B.A. Simmons, E.B. Cummings, and Y. Fintschenko, Dielectrophoretic concentration and separation of live and dead bacteria in an array of insulators. Analytical Chemistry, 2004. 76(6): p. 1571-1579.

111. Medoro, G., N. Manaresi, A. Leonardi, L. Altomare, M. Tartagni, and R. Guerrieri, A 
lab-on-a-chip for cell detection and manipulation. leee Sensors Journal, 2003. 3(3): p. 317-325.

112. Fiedler, S., S.G. Shirley, T. Schnelle, and G. Fuhr, Dielectrophoretic sorting of particles and cells in a microsystem. Analytical Chemistry, 1998. 70(9): p. 1909-1915.

113. Gascoyne, P.R.C., X.B. Wang, Y. Huang, and F.F. Becker, Dielectrophoretic separation of cancer cells from blood. leee Transactions on Industry Applications, 1997. 33(3): p. 670-678.

114. Gulati, G., Blood Cell Morpholohy Grading Guide. 2009.

115. Rasia, M. and A. Bollini, Red blood cell shape as a function of medium's ionic strength and pH. Biochimica Et Biophysica Acta-Biomembranes, 1998. 1372(2): p. 198-204.

116. Fischer, T.M., Shape memory of human red blood cells. Biophysical Journal, 2004. 86(5): p. 3304-3313.

117. Gedde, M.M., E.Y. Yang, and W.H. Huestis, SHAPE RESPONSE OF HUMAN ERYTHROCYTES TO ALTERED CELL PH. Blood, 1995. 86(4): p. 1595-1599.

118. Kinosita, K. and T.Y. Tsong, FORMATION AND RESEALING OF PORES OF CONTROLLED SIZES IN HUMAN ERYTHROCYTE-MEMBRANE. Nature, 1977. 268(5619): p. 438-441.

119. Tsong, T.Y. and E. Kingsley, HEMOLYSIS OF HUMAN ERYTHROCYTE INDUCED BY A RAPID TEMPERATURE JUMP. Journal of Biological Chemistry, 1975. 250(2): p. 786-789.

120. Movahed, S. and D.Q. Li, Microfluidics cell electroporation. Microfluidics and Nanofluidics, 2011. 10(4): p. 703-734.

121. Garcia-Sanchez, P., A. Ramos, A. Gonzalez, N.G. Green, and H. Morgan, Flow Reversal in Traveling-Wave Electrokinetics: An Analysis of Forces Due to lonic Concentration Gradients. Langmuir, 2009. 25(9): p. 4988-4997.

122. Turgeon, M.L., Clinical Hematology: Theory and Procedures. 2004: p. 100.

123. DeBruin, K.A. and W. Krassowska, Modeling electroporation in a single cell. I. Effects of field strength and rest potential. Biophysical Journal, 1999. 77(3): p. 1213-1224.

124. Chang, D.C. and T.S. Reese, CHANGES IN MEMBRANE-STRUCTURE INDUCED BY ELECTROPORATION AS REVEALED BY RAPID-FREEZING ELECTRON-MICROSCOPY. Biophysical Journal, 1990. 58(1): p. 1-12.

125. Bao, N., T.T. Le, J.X. Cheng, and C. Lu, Microfluidic electroporation of tumor and blood cells: observation of nucleus expansion and implications on selective analysis and purging of circulating tumor cells. Integrative Biology, 2010. 2(2-3): p. 113-120.

126. Moroz, V.V., A.M. Chernysh, E.K. Kozlova, P.Y. Borshegovskaya, U.A. Bliznjuk, R.M. Rysaeva, and O.Y. Gudkova, Comparison of red blood cell membrane microstructure after different physicochemical influences: Atomic force microscope research. Journal of Critical Care, 2010. 25(3).

127. Bilska, A.O., K.A. DeBruin, and W. Krassowska, Theoretical modeling of the effects of shock duration, frequency, and strength on the degree of electroporation. Bioelectrochemistry, 2000. 51(2): p. 133-143.

128. Djuzenova, C.S., U. Zimmermann, H. Frank, V.L. Sukhorukov, E. Richter, and G. Fuhr, Effect of 
medium conductivity and composition on the uptake of propidium iodide into electropermeabilized myeloma cells. Biochimica Et Biophysica Acta-Biomembranes, 1996. 1284(2): p. 143-152.

129. DeBruin, K.A. and W. Krassowska, Modeling electroporation in a single cell. II. Effects of ionic concentrations. Biophysical Journal, 1999. 77(3): p. 1225-1233.

130. Gencoglu, A., F. Camacho-Alanis, V.T. Nguyen, A. Nakano, A. Ros, and A.R. Minerick, Quantification of $\mathrm{pH}$ gradients and implications in insulator-based dielectrophoresis of biomolecules. Electrophoresis, 2011. 32(18): p. 2436-2447.

131. Ng, W.Y., Y.C. Lam, and I. Rodriguez, Experimental Verification of Faradaic Charging in AC Electrokinetics. Biomicrofluidics, 2009. 3(2).

132. Senftle, F.E., J.R. Grant, and F.P. Senftle, Low-voltage DC/AC electrolysis of water using porous graphite electrodes. Electrochimica Acta, 2010. 55(18): p. 5148-5153.

133. Utoh, J. and H. Harasaki, DAMAGE TO ERYTHROCYTES FROM LONG-TERM HEAT-STRESS. Clinical Science, 1992. 82(1): p. 9-11.

134. Sowemimo-Coker, S.O., Red blood cell hemolysis during processing. Transfusion Medicine Reviews, 2002. 16(1): p. 46-60.

135. Newman, J. and K.E. Thomas-Alyea, Electrochemical Systems. 2012: Wiley.

136. Lian, M., N. Islam, and J. Wu, AC electrothermal manipulation of conductive fluids and particles for lab-chip applications. let Nanobiotechnology, 2007. 1(3): p. 36-43.

137. Gagnon, Z.R. and H.C. Chang, Electrothermal ac electro-osmosis. Applied Physics Letters, 2009. 94(2).

138. García-Sánchez, P., A. Ramos, N.G. Green, and H. Morgan, Traveling-Wave Electrokinetic Micropumps: Velocity, Electrical Current, and Impedance Measurements. Langmuir, 2008. 24(17): p. 9361-9369.

139. Grahame, D.C., Electrode Processes and the Electrical Double Layer. Annual Review of Physical Chemistry, 1955. 6(1): p. 337-358.

140. Morgan, H. and N.G. Green, AC Electrokinetics: Colloids and Particles. 2003: Research Studies PressLtd.

141. Deen, W.M., Analysis of Transport Phenomena. 2 ed. 2011.

142. An, R., K. Massa, D.O. Wipf, and A.R. Minerick, Solution $p H$ change in non-uniform alternating current electric fields at frequencies above the electrode charging frequency. Biomicrofluidics, 2014. 8(6).

143. Garcia-Sanchez, P., A. Ramos, N.G. Green, and H. Morgan, Experiments on AC Electrokinetic Pumping of Liquids Using Arrays of Microelectrodes. Ieee Transactions on Dielectrics and Electrical Insulation, 2006. 13(3): p. 670-677.

144. Bard, A.J. and L.R. Faulkner, Electrochemical Methods: Fundamentals and Applications. 2000: Wiley.

145. Bruus, H., Theoretical Microfluidics. 2008.

146. Gonzalez, A., A. Ramos, P. Garcia-Sanchez, and A. Castellanos, Effect of the Combined Action 
of Faradaic Currents and Mobility Differences in AC Electro-osmosis. Physical Review E, 2010. 81(1).

147. Linkov, V., Electrolysis. 2012.

148. Studer, V., A. Pepin, Y. Chen, and A. Ajdari, An Integrated AC Electrokinetic Pump in A Microfluidic Loop for Fast and Tunable Flow Control. Analyst, 2004. 129(10): p. 944-949.

149. Contento, N.M. and P.W. Bohn, Tunable Electrochemical pH Modulation in A Microchannel Monitored via The Proton-coupled Electro-oxidation of Hydroquinone. Biomicrofluidics, 2014. 8(4): p. - .

150. Cheng, L.J. and H.C. Chang, Switchable pH Actuators and 3D Integrated Salt Bridges as New Srategies for Reconfigurable Microfluidic Free-flow Electrophoretic Separation. Lab on a Chip, 2014. 14(5): p. 979-987.

151. Cheng, L.-J. and H.-C. Chang, Microscale pH Regulation by Splitting Water. Biomicrofluidics, 2011. 5(4): p. -.

152. Xie, C.G., J. Bostaph, and J. Pavio, Development of A 2 W Direct Methanol Fuel Cell Power Source. Journal of Power Sources, 2004. 136(1): p. 55-65.

153. John O'M. Bockeris and A.K.N. Reddy, Modern Electrochemistry 1 lonics. 2 ed. 1998.

154. Manuel Shaw and A.E. Remick, Studies on Alternating Current Electrolysis I. The Nature of Polarization Capacity and Polarization Resistance. J. Electrochem. Soc, 1950. 97(10): p. 324-334.

155. Jacob Silverman and A.E. Remick, Studies on Alternating current electrolysis. 2. Oscillographic studies. J. Electrochem. Soc, 1950. 97(10): p. 335-345.

156. Donald D. Bump and A.E. Remick, Studies on Alternating Current Electrolysis: V. Double Layer Resistance and Electrode Area as Factors in Electrode Kinetics. J. Electrochem. Soc, 1964. 111(8): p. 981-988.

157. A. Edward Remick and H.W. Mccormick, Studies on Alternating - Current Electrolysis: III . Effects of Concentration on Polarization Capacity and Polarization Resistance. J. Electrochem. Soc, 1955. 102(9): p. 534-544.

158. Ren, Y.K., H.Y. Jiang, H.K. Yang, A. Ramos, and P. Garcia-Sanchez, Electrical Manipulation of Electrolytes with Conductivity Gradients in Microsystems. Journal of Electrostatics, 2009. 67(2-3): p. 372-376.

159. Barz, D.P.J., Comprehensive Model of Electrokinetic Flow and Migration in Microchannels with Conductivity Gradients. Microfluidics and Nanofluidics, 2009. 7(2): p. 249-265.

160. Desmond, M., N. Mavrogiannis, and Z. Gagnon, Maxwell-Wagner Polarization and Frequency-Dependent Injection at Aqueous Electrical Interfaces. Physical Review Letters, 2012. 109(18).

161. Khair, A.S. and T.M. Squires, Surprising Consequences of Ion Conservation in Electro-osmosis over A Surface Charge Discontinuity. Journal of Fluid Mechanics, 2008. 615: p. 323-334.

162. Chang, C.C., C.P. Yeh, and R.J. Yang, lon Concentration Polarization Near Microchannel-nanochannel Interfaces: Effect of pH Value. Electrophoresis, 2012. 33(5): p. 
758-764.

163. Yota, J., H. Shen, and R. Ramanathan, Characterization of Atomic Layer Deposition HfO2, Al2O3, and Plasma-enhanced Chemical Vapor Deposition Si3N4 as Metal-insulator-metal Capacitor Dielectric for GaAs HBT Technology. Journal of Vacuum Science \& Technology A, 2013. 31(1).

164. Bockris, J.O.M., A.K.N. Reddy, M. Gamboa-Aldeco, and M.E. Gamboa-Aldeco, Modern Electrochemistry. 2000: Springer.

165. Jackson, M.J., Microfabrication and Nanomanufacturing. 2005: Taylor \& Francis.

166. Chen, C.H., H. Lin, S.K. Lele, and J.G. Santiago, Convective and Absolute Electrokinetic Instability with Conductivity Gradients. Journal of Fluid Mechanics, 2005. 524: p. 263-303.

167. Pascall, A.J. and T.M. Squires, Electrokinetics at Liquid/Liquid Interfaces. Journal of Fluid Mechanics, 2011. 684: p. 163-191.

168. Golovnev, A. and S. Trimper, Analytical Solution of The PNP Equations at AC Applied Voltage. Physics Letters A, 2012. 376(16): p. 1391-1395. 


\section{Appendix PROOFS OF PERMISSION TO REPRODUCE PUBLISHED MATERIAL}

The proofs for the reproduced material are attached as follows:

1. Permission for reproduced figure 5 in chapter 1

2. Permission for reproduced figure 10 in chapter 2

3. Permission for reproduced figure 11 and 12 in chapter 2

4. Permission for reproduced figure 13 in chapter 2

5. Permission for reproduced article in chapter 3

6. Permission for reproduced article in chapter 4 


\section{SPRINGER LICENSE}

\section{TERMS AND CONDITIONS}

Mar 29, 2015

This is a License Agreement between Ran An ("You") and Springer ("Springer") provided by Copyright

Clearance Center ("CCC"). The license consists of your order details, the terms and conditions provided by Springer, and the payment terms and conditions.

All payments must be made in full to CCC. For payment instructions, please see information listed at the bottom of this form.

License Number

3598331004343

License date

Mar 29, 2015

Licensed content publisher

Springer

Licensed content publication

Microfluids and Nanofluids

Licensed content title

Microfluidic flow reversal at low frequency by AC electrothermal effect

Licensed content author

Meng Lian

Licensed content date

Jan 1, 2009 


\section{Volume number}

7

Issue number

6

Type of Use

Thesis/Dissertation

Portion

Figures

Author of this Springer article

No

Order reference number

None

Original figure numbers

Figure 6

Title of your thesis / dissertation

ELECTROCHEMICAL PROCESSES IN MICROFLUIDICS SYSTEMS UNDER AC ELECTRIC FIELDS

Expected completion date

May 2015

Estimated size(pages) 
Total

0.00 USD

Terms and Conditions

Introduction

The publisher for this copyrighted material is Springer Science + Business Media. By clicking "accept" in connection with completing this licensing transaction, you agree that the following terms and conditions apply to this transaction (along with the Billing and Payment terms and conditions established by Copyright Clearance Center, Inc. ("CCC"), at the time that you opened your Rightslink account and that are available at any time at http://myaccount.copyright.com).

Limited License

With reference to your request to reprint in your thesis material on which Springer Science and Business Media control the copyright, permission is granted, free of charge, for the use indicated in your enquiry.

Licenses are for one-time use only with a maximum distribution equal to the number that you identified in the licensing process.

This License includes use in an electronic form, provided its password protected or on the university's intranet or repository, including UMI (according to the definition at the Sherpa website: http://www.sherpa.ac.uk/romeo/).

For any other electronic use, please contact Springer at (permissions.dordrecht@springer.com or permissions.heidelberg@springer.com).

The material can only be used for the purpose of defending your thesis limited to university-use only. If the thesis is going to be published, permission needs to be re-obtained (selecting "book/textbook" as the type of use).

Although Springer holds copyright to the material and is entitled to negotiate on rights, this license is only valid, subject to a courtesy information to the author (address is given with the article/chapter) and provided it concerns original material which does not carry references to other sources (if material in question appears with credit to another source, authorization from that source is required as well).

Permission free of charge on this occasion does not prejudice any rights we might have to charge for reproduction of our copyrighted material in the future.

Altering/Modifying Material: Not Permitted 
You may not alter or modify the material in any manner. Abbreviations, additions, deletions and/or any other alterations shall be made only with prior written authorization of the author(s) and/or Springer Science + Business Media. (Please contact Springer at (permissions.dordrecht@springer.com or permissions.heidelberg@springer.com)

\section{Reservation of Rights}

Springer Science + Business Media reserves all rights not specifically granted in the combination of (i) the license details provided by you and accepted in the course of this licensing transaction, (ii) these terms and conditions and (iii) CCC's Billing and Payment terms and conditions.

\section{Copyright Notice:Disclaimer}

You must include the following copyright and permission notice in connection with any reproduction of the licensed material: $\quad$ "Springer and the original publisher /journal title, volume, year of publication, page, chapter/article title, name(s) of author(s), figure number(s), original copyright notice) is given to the publication in which the material was originally published, by adding; with kind permission from Springer Science and Business Media"

Warranties: None

Example 1: Springer Science + Business Media makes no representations or warranties with respect to the licensed material.

Example 2: Springer Science + Business Media makes no representations or warranties with respect to the licensed material and adopts on its own behalf the limitations and disclaimers established by CCC on its behalf in its Billing and Payment terms and conditions for this licensing transaction.

Indemnity

You hereby indemnify and agree to hold harmless Springer Science + Business Media and CCC, and their respective officers, directors, employees and agents, from and against any and all claims arising out of your use of the licensed material other than as specifically authorized pursuant to this license.

\section{No Transfer of License}

This license is personal to you and may not be sublicensed, assigned, or transferred by you to any other person without Springer Science + Business Media's written permission.

\section{No Amendment Except in Writing}

This license may not be amended except in a writing signed by both parties (or, in the case of Springer Science + Business Media, by CCC on Springer Science + Business Media's behalf). 
Objection to Contrary Terms

Springer Science + Business Media hereby objects to any terms contained in any purchase order,

acknowledgment, check endorsement or other writing prepared by you, which terms are inconsistent with these

terms and conditions or CCC's Billing and Payment terms and conditions. These terms and conditions, together with CCC's Billing and Payment terms and conditions (which are incorporated herein), comprise the entire agreement between you and Springer Science + Business Media (and CCC) concerning this licensing transaction. In the event of any conflict between your obligations established by these terms and conditions and those established by CCC's Billing and Payment terms and conditions, these terms and conditions shall control.

Jurisdiction

All disputes that may arise in connection with this present License, or the breach thereof, shall be settled exclusively by arbitration, to be held in The Netherlands, in accordance with Dutch law, and to be conducted under the Rules of the 'Netherlands Arbitrage Instituut' (Netherlands Institute of Arbitration).OR:

All disputes that may arise in connection with this present License, or the breach thereof, shall be settled exclusively by arbitration, to be held in the Federal Republic of Germany, in accordance with German law.

Other terms and conditions:

v1.3

Questions? customercare@copyright.com or +1-855-239-3415 (toll free in the US) or +1-978-646-2777.

Gratis licenses (referencing $\$ 0$ in the Total field) are free. Please retain this printable license for your reference. No payment is required.

2. This License Agreement is between \{Requestor Name\} ("You") and The Royal Society of Chemistry ("RSC") provided by the Copyright Clearance Center (“CCC"). The license consists of your order details, the terms and conditions provided by the Royal Society of Chemistry, and the payment terms and conditions.

\section{RSC / TERMS AND CONDITIONS}

\section{INTRODUCTION}

The publisher for this copyrighted material is The Royal Society of Chemistry. By clicking "accept" in connection with completing this licensing transaction, you agree that the following terms and conditions apply to this transaction (along with the Billing and Payment terms and conditions established by CCC, at the time that you opened your RightsLink account and that are available at any time at . 


\section{LICENSE GRANTED}

The RSC hereby grants you a non-exclusive license to use the aforementioned material anywhere in the world subject to the terms and conditions indicated herein. Reproduction of the material is confined to the purpose and/or media for which permission is hereby given.

\section{RESERVATION OF RIGHTS}

The RSC reserves all rights not specifically granted in the combination of (i) the license details provided by your and accepted in the course of this licensing transaction; (ii) these terms and conditions; and (iii) CCC's Billing and Payment terms and conditions.

\section{REVOCATION}

The RSC reserves the right to revoke this license for any reason, including, but not limited to, advertising and promotional uses of RSC content, third party usage, and incorrect source figure attribution.

\section{THIRD-PARTY MATERIAL DISCLAIMER}

If part of the material to be used (for example, a figure) has appeared in the RSC publication with credit to another source, permission must also be sought from that source. If the other source is another RSC publication these details should be included in your RightsLink request. If the other source is a third party, permission must be obtained from the third party. The RSC disclaims any responsibility for the reproduction you make of items owned by a third party.

\section{PAYMENT OF FEE}

If the permission fee for the requested material is waived in this instance, please be advised that any future requests for the reproduction of RSC materials may attract a fee.

\section{ACKNOWLEDGEMENT}

The reproduction of the licensed material must be accompanied by the following acknowledgement:

Reproduced (“Adapted” or “in part”) from \{Reference Citation\} (or Ref XX) with permission of The Royal Society of Chemistry.

If the licensed material is being reproduced from New Journal of Chemistry (NJC), Photochemical \& Photobiological Sciences (PPS) or Physical Chemistry Chemical Physics (PCCP) you must include one of the following acknowledgements:

For figures originally published in NJC:

Reproduced (“Adapted” or "in part”) from \{Reference Citation\} (or Ref XX) with permission of The Royal Society of Chemistry (RSC) on behalf of the European Society for Photobiology, the European Photochemistry Association and the RSC. 
For figures originally published in PPS:

Reproduced (“Adapted” or "in part”) from \{Reference Citation\} (or Ref XX) with permission of The Royal Society of Chemistry (RSC) on behalf of the Centre National de la Recherche Scientifique (CNRS) and the RSC.

For figures originally published in PCCP:

Reproduced (“Adapted” or "in part”) from \{Reference Citation\} (or Ref XX) with permission of the PCCP Owner Societies.

\section{HYPERTEXT LINKS}

With any material which is being reproduced in electronic form, you must include a hypertext link to the original RSC article on the RSC's website. The recommended form for the hyperlink is http://dx.doi.org/10.1039/DOI suffix, for example in the link http://dx.doi.org/10.1039/b110420a the DOI suffix is 'b110420a'. To find the relevant DOI suffix for the RSC article in question, go to the Journals section of the website and locate the article in the list of papers for the volume and issue of your specific journal. You will find the DOI suffix quoted there.

\section{LICENSE CONTINGENT ON PAYMENT}

While you may exercise the rights licensed immediately upon issuance of the license at the end of the licensing process for the transaction, provided that you have disclosed complete and accurate details of your proposed use, no license is finally effective unless and until full payment is received from you (by CCC) as provided in CCC's Billing and Payment terms and conditions. If full payment is not received on a timely basis, then any license preliminarily granted shall be deemed automatically revoked and shall be void as if never granted. Further, in the event that you breach any of these terms and conditions or any of CCC's Billing and Payment terms and conditions, the license is automatically revoked and shall be void as if never granted. Use of materials as described in a revoked license, as well as any use of the materials beyond the scope of an unrevoked license, may constitute copyright infringement and the RSC reserves the right to take any and all action to protect its copyright in the materials.

\section{WARRANTIES}

The RSC makes no representations or warranties with respect to the licensed material.

\section{INDEMNITY}

You hereby indemnify and agree to hold harmless the RSC and the CCC, and their respective officers, directors, trustees, employees and agents, from and against any and all claims arising out of your use of the licensed material other than as specifically authorized pursuant to this licence.

\section{NO TRANSFER OF LICENSE}

This license is personal to you or your publisher and may not be sublicensed, assigned, or transferred by you to any other person without the RSC's written permission. 


\section{NO AMENDMENT EXCEPT IN WRITING}

This license may not be amended except in a writing signed by both parties (or, in the case of "Other Conditions, v1.2", by CCC on the RSC's behalf).

\section{OBJECTION TO CONTRARY TERMS}

You hereby acknowledge and agree that these terms and conditions, together with CCC's Billing and Payment terms and conditions (which are incorporated herein), comprise the entire agreement between you and the RSC (and CCC) concerning this licensing transaction, to the exclusion of all other terms and conditions, written or verbal, express or implied (including any terms contained in any purchase order, acknowledgment, check endorsement or other writing prepared by you). In the event of any conflict between your obligations established by these terms and conditions and those established by CCC's Billing and Payment terms and conditions, these terms and conditions shall control.

\section{JURISDICTION}

This license transaction shall be governed by and construed in accordance with the laws of the District of Columbia. You hereby agree to submit to the jurisdiction of the courts located in the District of Columbia for purposes of resolving any disputes that may arise in connection with this licensing transaction.

\section{LIMITED LICENSE}

The following terms and conditions apply to specific license types:

Translation

This permission is granted for non-exclusive world English rights only unless your license was granted for translation rights. If you licensed translation rights you may only translate this content into the languages you requested. A professional translator must perform all translations and reproduce the content word for word preserving the integrity of the article.

Intranet

If the licensed material is being posted on an Intranet, the Intranet is to be password-protected and made available only to bona fide students or employees only. All content posted to the Intranet must maintain the copyright information line on the bottom of each image. You must also fully reference the material and include a hypertext link as specified above.

Copies of Whole Articles

All copies of whole articles must maintain, if available, the copyright information line on the bottom of each page.

Other Conditions

v1.2 
Gratis licenses (referencing $\$ 0$ in the Total field) are free. Please retain this printable license for your reference. No payment is required.

If you would like to pay for this license now, please remit this license along with yourpayment made payable to "COPYRIGHT CLEARANCE CENTER" otherwise you will be invoiced within 48 hours of the license date. Payment should be in the form of a check or money order referencing your account number and this invoice number \{Invoice Number\}.

Once you receive your invoice for this order, you may pay your invoice by credit card.

Please follow instructions provided at that time.

Make Payment To:

Copyright Clearance Center

Dept 001

P.O. Box 843006

Boston, MA 02284-3006

For suggestions or comments regarding this order, contact Rightslink Customer Support:

customercare@copyright.com or +1-855-239-3415 (toll free in the US) or +1-978-646-2777.

3. This License Agreement is between \{Requestor Name\} ("You") and The Royal Society of Chemistry ("RSC") provided by the Copyright Clearance Center ("CCC"). The license consists of your order details, the terms and conditions provided by the Royal Society of Chemistry, and the payment terms and conditions.

RSC / TERMS AND CONDITIONS

\section{INTRODUCTION}

The publisher for this copyrighted material is The Royal Society of Chemistry. By clicking "accept" in connection with completing this licensing transaction, you agree that the following terms and conditions apply to this transaction (along with the Billing and Payment terms and conditions established by CCC, at the time that you opened your RightsLink account and that are available at any time at .

\section{LICENSE GRANTED}

The RSC hereby grants you a non-exclusive license to use the aforementioned material anywhere in the world subject to the terms and conditions indicated herein. Reproduction of the material is confined to the purpose and/or media for which permission is hereby given.

\section{RESERVATION OF RIGHTS}

The RSC reserves all rights not specifically granted in the combination of (i) the license details provided by your 
and accepted in the course of this licensing transaction; (ii) these terms and conditions; and (iii) CCC's Billing and Payment terms and conditions.

\section{REVOCATION}

The RSC reserves the right to revoke this license for any reason, including, but not limited to, advertising and promotional uses of RSC content, third party usage, and incorrect source figure attribution.

\section{THIRD-PARTY MATERIAL DISCLAIMER}

If part of the material to be used (for example, a figure) has appeared in the RSC publication with credit to another source, permission must also be sought from that source. If the other source is another RSC publication these details should be included in your RightsLink request. If the other source is a third party, permission must be obtained from the third party. The RSC disclaims any responsibility for the reproduction you make of items owned by a third party.

\section{PAYMENT OF FEE}

If the permission fee for the requested material is waived in this instance, please be advised that any future requests for the reproduction of RSC materials may attract a fee.

\section{ACKNOWLEDGEMENT}

The reproduction of the licensed material must be accompanied by the following acknowledgement:

Reproduced (“Adapted” or "in part”) from \{Reference Citation\} (or Ref XX) with permission of The Royal Society of Chemistry.

If the licensed material is being reproduced from New Journal of Chemistry (NJC), Photochemical \& Photobiological Sciences (PPS) or Physical Chemistry Chemical Physics (PCCP) you must include one of the following acknowledgements:

For figures originally published in NJC:

Reproduced (“Adapted" or "in part”) from \{Reference Citation $\}$ (or Ref XX) with permission of The Royal Society of Chemistry (RSC) on behalf of the European Society for Photobiology, the European Photochemistry Association and the RSC.

For figures originally published in PPS:

Reproduced (“Adapted” or "in part”) from \{Reference Citation\} (or Ref XX) with permission of The Royal Society of Chemistry (RSC) on behalf of the Centre National de la Recherche Scientifique (CNRS) and the RSC.

For figures originally published in PCCP: 
Reproduced (“Adapted” or "in part”) from \{Reference Citation\} (or Ref XX) with permission of the PCCP Owner Societies.

\section{HYPERTEXT LINKS}

With any material which is being reproduced in electronic form, you must include a hypertext link to the original RSC article on the RSC's website. The recommended form for the hyperlink is http://dx.doi.org/10.1039/DOI suffix, for example in the link http://dx.doi.org/10.1039/b110420a the DOI suffix is 'b110420a'. To find the relevant DOI suffix for the RSC article in question, go to the Journals section of the website and locate the article in the list of papers for the volume and issue of your specific journal. You will find the DOI suffix quoted there.

\section{LICENSE CONTINGENT ON PAYMENT}

While you may exercise the rights licensed immediately upon issuance of the license at the end of the licensing process for the transaction, provided that you have disclosed complete and accurate details of your proposed use, no license is finally effective unless and until full payment is received from you (by CCC) as provided in CCC's Billing and Payment terms and conditions. If full payment is not received on a timely basis, then any license preliminarily granted shall be deemed automatically revoked and shall be void as if never granted. Further, in the event that you breach any of these terms and conditions or any of CCC's Billing and Payment terms and conditions, the license is automatically revoked and shall be void as if never granted. Use of materials as described in a revoked license, as well as any use of the materials beyond the scope of an unrevoked license, may constitute copyright infringement and the RSC reserves the right to take any and all action to protect its copyright in the materials.

\section{WARRANTIES}

The RSC makes no representations or warranties with respect to the licensed material.

\section{INDEMNITY}

You hereby indemnify and agree to hold harmless the RSC and the CCC, and their respective officers, directors, trustees, employees and agents, from and against any and all claims arising out of your use of the licensed material other than as specifically authorized pursuant to this licence.

\section{NO TRANSFER OF LICENSE}

This license is personal to you or your publisher and may not be sublicensed, assigned, or transferred by you to any other person without the RSC's written permission.

\section{NO AMENDMENT EXCEPT IN WRITING}

This license may not be amended except in a writing signed by both parties (or, in the case of "Other Conditions, v1.2", by CCC on the RSC's behalf).

\section{OBJECTION TO CONTRARY TERMS}

You hereby acknowledge and agree that these terms and conditions, together with CCC's Billing and Payment 
terms and conditions (which are incorporated herein), comprise the entire agreement between you and the RSC (and CCC) concerning this licensing transaction, to the exclusion of all other terms and conditions, written or verbal, express or implied (including any terms contained in any purchase order, acknowledgment, check endorsement or other writing prepared by you). In the event of any conflict between your obligations established by these terms and conditions and those established by CCC's Billing and Payment terms and conditions, these terms and conditions shall control.

\section{JURISDICTION}

This license transaction shall be governed by and construed in accordance with the laws of the District of Columbia. You hereby agree to submit to the jurisdiction of the courts located in the District of Columbia for purposes of resolving any disputes that may arise in connection with this licensing transaction.

\section{LIMITED LICENSE}

The following terms and conditions apply to specific license types:

Translation

This permission is granted for non-exclusive world English rights only unless your license was granted for translation rights. If you licensed translation rights you may only translate this content into the languages you requested. A professional translator must perform all translations and reproduce the content word for word preserving the integrity of the article.

Intranet

If the licensed material is being posted on an Intranet, the Intranet is to be password-protected and made available only to bona fide students or employees only. All content posted to the Intranet must maintain the copyright information line on the bottom of each image. You must also fully reference the material and include a hypertext link as specified above.

Copies of Whole Articles

All copies of whole articles must maintain, if available, the copyright information line on the bottom of each page.

Other Conditions

$\mathrm{v} 1.2$

Gratis licenses (referencing $\$ 0$ in the Total field) are free. Please retain this printable license for your reference. No payment is required.

If you would like to pay for this license now, please remit this license along with yourpayment made payable to "COPYRIGHT CLEARANCE CENTER" otherwise you will be invoiced within 48 hours of the license date. 
Payment should be in the form of a check or money order referencing your account number and this invoice number \{Invoice Number\}.

Once you receive your invoice for this order, you may pay your invoice by credit card.

Please follow instructions provided at that time.

Make Payment To:

Copyright Clearance Center

Dept 001

P.O. Box 843006

Boston, MA 02284-3006

For suggestions or comments regarding this order, contact Rightslink Customer Support:

customercare@copyright.com or +1-855-239-3415 (toll free in the US) or +1-978-646-2777.

\section{PERMISSION/LICENSE IS GRANTED FOR YOUR ORDER AT NO CHARGE}

This type of permission/license, instead of the standard Terms \& Conditions, is sent to you because no fee is being charged for your order. Please note the following:

- Permission is granted for your request in both print and electronic formats, and translations.

- If figures and/or tables were requested, they may be adapted or used in part.

- $\quad$ Please print this page for your records and send a copy of it to your publisher/graduate school.

- Appropriate credit for the requested material should be given as follows: "Reprinted (adapted) with permission from (COMPLETE REFERENCE CITATION). Copyright (YEAR) American Chemical Society." Insert appropriate information in place of the capitalized words.

- One-time permission is granted only for the use specified in your request. No additional uses are granted (such as derivative works or other editions). For any other uses, please submit a new request.

4. This License Agreement is between \{Requestor Name\} ("You") and The Royal Society of Chemistry ("RSC") provided by the Copyright Clearance Center ("CCC"). The license consists of your order details, the terms and conditions provided by the Royal Society of Chemistry, and the payment terms and conditions.

RSC / TERMS AND CONDITIONS

INTRODUCTION

The publisher for this copyrighted material is The Royal Society of Chemistry. By clicking "accept" in 
connection with completing this licensing transaction, you agree that the following terms and conditions apply to this transaction (along with the Billing and Payment terms and conditions established by CCC, at the time that you opened your RightsLink account and that are available at any time at .

\section{LICENSE GRANTED}

The RSC hereby grants you a non-exclusive license to use the aforementioned material anywhere in the world subject to the terms and conditions indicated herein. Reproduction of the material is confined to the purpose and/or media for which permission is hereby given.

\section{RESERVATION OF RIGHTS}

The RSC reserves all rights not specifically granted in the combination of (i) the license details provided by your and accepted in the course of this licensing transaction; (ii) these terms and conditions; and (iii) CCC's Billing and Payment terms and conditions.

\section{REVOCATION}

The RSC reserves the right to revoke this license for any reason, including, but not limited to, advertising and promotional uses of RSC content, third party usage, and incorrect source figure attribution.

\section{THIRD-PARTY MATERIAL DISCLAIMER}

If part of the material to be used (for example, a figure) has appeared in the RSC publication with credit to another source, permission must also be sought from that source. If the other source is another RSC publication these details should be included in your RightsLink request. If the other source is a third party, permission must be obtained from the third party. The RSC disclaims any responsibility for the reproduction you make of items owned by a third party.

\section{PAYMENT OF FEE}

If the permission fee for the requested material is waived in this instance, please be advised that any future requests for the reproduction of RSC materials may attract a fee.

\section{ACKNOWLEDGEMENT}

The reproduction of the licensed material must be accompanied by the following acknowledgement:

Reproduced (“Adapted" or "in part”) from \{Reference Citation\} (or Ref XX) with permission of The Royal Society of Chemistry.

If the licensed material is being reproduced from New Journal of Chemistry (NJC), Photochemical \& Photobiological Sciences (PPS) or Physical Chemistry Chemical Physics (PCCP) you must include one of the following acknowledgements: 
For figures originally published in NJC:

Reproduced (“Adapted” or "in part”) from \{Reference Citation\} (or Ref XX) with permission of The Royal Society of Chemistry (RSC) on behalf of the European Society for Photobiology, the European Photochemistry Association and the RSC.

For figures originally published in PPS:

Reproduced (“Adapted” or "in part”) from \{Reference Citation\} (or Ref XX) with permission of The Royal Society of Chemistry (RSC) on behalf of the Centre National de la Recherche Scientifique (CNRS) and the RSC.

For figures originally published in PCCP:

Reproduced (“Adapted” or "in part”) from \{Reference Citation\} (or Ref XX) with permission of the PCCP Owner Societies.

\section{HYPERTEXT LINKS}

With any material which is being reproduced in electronic form, you must include a hypertext link to the original RSC article on the RSC's website. The recommended form for the hyperlink is

http://dx.doi.org/10.1039/DOI suffix, for example in the link http://dx.doi.org/10.1039/b110420a the DOI suffix is 'b110420a'. To find the relevant DOI suffix for the RSC article in question, go to the Journals section of the website and locate the article in the list of papers for the volume and issue of your specific journal. You will find the DOI suffix quoted there.

\section{LICENSE CONTINGENT ON PAYMENT}

While you may exercise the rights licensed immediately upon issuance of the license at the end of the licensing process for the transaction, provided that you have disclosed complete and accurate details of your proposed use, no license is finally effective unless and until full payment is received from you (by CCC) as provided in CCC's Billing and Payment terms and conditions. If full payment is not received on a timely basis, then any license preliminarily granted shall be deemed automatically revoked and shall be void as if never granted. Further, in the event that you breach any of these terms and conditions or any of CCC's Billing and Payment terms and conditions, the license is automatically revoked and shall be void as if never granted. Use of materials as described in a revoked license, as well as any use of the materials beyond the scope of an unrevoked license, may constitute copyright infringement and the RSC reserves the right to take any and all action to protect its copyright in the materials.

\section{WARRANTIES}


The RSC makes no representations or warranties with respect to the licensed material.

\section{INDEMNITY}

You hereby indemnify and agree to hold harmless the RSC and the CCC, and their respective officers, directors, trustees, employees and agents, from and against any and all claims arising out of your use of the licensed material other than as specifically authorized pursuant to this licence.

\section{NO TRANSFER OF LICENSE}

This license is personal to you or your publisher and may not be sublicensed, assigned, or transferred by you to any other person without the RSC's written permission.

\section{NO AMENDMENT EXCEPT IN WRITING}

This license may not be amended except in a writing signed by both parties (or, in the case of "Other Conditions, v1.2", by CCC on the RSC's behalf).

\section{OBJECTION TO CONTRARY TERMS}

You hereby acknowledge and agree that these terms and conditions, together with CCC's Billing and Payment terms and conditions (which are incorporated herein), comprise the entire agreement between you and the RSC (and CCC) concerning this licensing transaction, to the exclusion of all other terms and conditions, written or verbal, express or implied (including any terms contained in any purchase order, acknowledgment, check endorsement or other writing prepared by you). In the event of any conflict between your obligations established by these terms and conditions and those established by CCC's Billing and Payment terms and conditions, these terms and conditions shall control.

\section{JURISDICTION}

This license transaction shall be governed by and construed in accordance with the laws of the District of Columbia. You hereby agree to submit to the jurisdiction of the courts located in the District of Columbia for purposes of resolving any disputes that may arise in connection with this licensing transaction.

\section{LIMITED LICENSE}

The following terms and conditions apply to specific license types:

Translation

This permission is granted for non-exclusive world English rights only unless your license was granted for translation rights. If you licensed translation rights you may only translate this content into the languages you requested. A professional translator must perform all translations and reproduce the content word for word preserving the integrity of the article.

Intranet 
If the licensed material is being posted on an Intranet, the Intranet is to be password-protected and made available only to bona fide students or employees only. All content posted to the Intranet must maintain the copyright information line on the bottom of each image. You must also fully reference the material and include a hypertext link as specified above.

Copies of Whole Articles

All copies of whole articles must maintain, if available, the copyright information line on the bottom of each page.

Other Conditions

$\mathrm{v} 1.2$

Gratis licenses (referencing $\$ 0$ in the Total field) are free. Please retain this printable license for your reference. No payment is required.

If you would like to pay for this license now, please remit this license along with yourpayment made payable to "COPYRIGHT CLEARANCE CENTER" otherwise you will be invoiced within 48 hours of the license date. Payment should be in the form of a check or money order referencing your account number and this invoice number $\{$ Invoice Number\}.

Once you receive your invoice for this order, you may pay your invoice by credit card.

Please follow instructions provided at that time.

Make Payment To:

Copyright Clearance Center

Dept 001

P.O. Box 843006

Boston, MA 02284-3006

For suggestions or comments regarding this order, contact Rightslink Customer Support:

customercare@copyright.com or +1-855-239-3415 (toll free in the US) or +1-978-646-2777.

5. AIP Publishing LLC -- Terms and Conditions: Permissions Uses

AIP Publishing LLC ("AIPP"") hereby grants to you the non-exclusive right and license to use and/or distribute the Material according to the use specified in your order, on a one-time basis, for the specified term, with a maximum distribution equal to the number that you have ordered. Any links or other content accompanying the Material are not the subject of this license. 
1. You agree to include the following copyright and permission notice with the reproduction of the Material:"Reprinted with permission from [FULL CITATION]. Copyright [PUBLICATION YEAR], AIP Publishing LLC." For an article, the copyright and permission notice must be printed on the first page of the article or book chapter. For photographs, covers, or tables, the copyright and permission notice may appear with the Material, in a footnote, or in the reference list.

2. If you have licensed reuse of a figure, photograph, cover, or table, it is your responsibility to ensure that the material is original to AIPP and does not contain the copyright of another entity, and that the copyright notice of the figure, photograph, cover, or table does not indicate that it was reprinted by AIPP, with permission, from another source. Under no circumstances does AIPP, purport or intend to grant permission to reuse material to which it does not hold copyright.

3. You may not alter or modify the Material in any manner. You may translate the Material into another language only if you have licensed translation rights. You may not use the Material for promotional purposes. AIPP reserves all rights not specifically granted herein.

4. The foregoing license shall not take effect unless and until AIPP or its agent, Copyright Clearance Center, receives the Payment in accordance with Copyright Clearance Center Billing and Payment Terms and Conditions, which are incorporated herein by reference.

5. AIPP or the Copyright Clearance Center may, within two business days of granting this license, revoke the license for any reason whatsoever, with a full refund payable to you. Should you violate the terms of this license at any time, AIPP, AIP Publishing LLC, or Copyright Clearance Center may revoke the license with no refund to you. Notice of such revocation will be made using the contact information provided by you. Failure to receive such notice will not nullify the revocation.

6. AIPP makes no representations or warranties with respect to the Material. You agree to indemnify and hold harmless AIPP, AIP Publishing LLC, and their officers, directors, employees or agents from and against any and all claims arising out of your use of the Material other than as specifically authorized herein.

7. The permission granted herein is personal to you and is not transferable or assignable without the prior written permission of AIPP. This license may not be amended except in a writing signed by the party to be charged.

8. If purchase orders, acknowledgments or check endorsements are issued on any forms containing terms and conditions which are inconsistent with these provisions, such inconsistent terms and conditions shall be of no force and effect. This document, including the CCC Billing and Payment Terms and Conditions, shall be the entire agreement between the parties relating to the subject matter hereof.

This Agreement shall be governed by and construed in accordance with the laws of the State of New York. Both parties hereby submit to the jurisdiction of the courts of New York County for purposes of resolving any disputes that may arise hereunder.

6. License Number 
Order Date

Mar 29, 2015

Publisher

AIP Publishing LLC

Publication

Biomicrofluidics

Article Title

Solution $\mathrm{pH}$ change in non-uniform alternating current electric fields at frequencies above the electrode charging frequency

Author

Ran An,Katherine Massa,David O. Wipf, et al.

Online Publication Date

Dec 19, 2014

Volume number

8

Issue number

6

Type of Use

Thesis/Dissertation 
Requestor type

Author (original article)

Format

Print and electronic

Portion

Excerpt ( $>800$ words)

Will you be translating?

No

Title of your thesis / dissertation

ELECTROCHEMICAL PROCESSES IN MICROFLUIDICS SYSTEMS UNDER AC ELECTRIC FIELDS

Expected completion date

May 2015

Estimated size (number of pages)

200

Total

0.00 USD

Terms and Conditions

AIP Publishing LLC -- Terms and Conditions: Permissions Uses

AIP Publishing LLC ("AIPP"') hereby grants to you the non-exclusive right and license to use and/or distribute the Material according to the use specified in your order, on a one-time basis, for the specified term, with a maximum distribution equal to the number that you have ordered. Any links or other content accompanying the 
Material are not the subject of this license.

You agree to include the following copyright and permission notice with the reproduction of the Material:"Reprinted with permission from [FULL CITATION]. Copyright [PUBLICATION YEAR], AIP Publishing LLC." For an article, the copyright and permission notice must be printed on the first page of the article or book chapter. For photographs, covers, or tables, the copyright and permission notice may appear with the Material, in a footnote, or in the reference list.

If you have licensed reuse of a figure, photograph, cover, or table, it is your responsibility to ensure that the material is original to AIPP and does not contain the copyright of another entity, and that the copyright notice of the figure, photograph, cover, or table does not indicate that it was reprinted by AIPP, with permission, from another source. Under no circumstances does AIPP, purport or intend to grant permission to reuse material to which it does not hold copyright.

You may not alter or modify the Material in any manner. You may translate the Material into another language only if you have licensed translation rights. You may not use the Material for promotional purposes. AIPP reserves all rights not specifically granted herein.

The foregoing license shall not take effect unless and until AIPP or its agent, Copyright Clearance Center, receives the Payment in accordance with Copyright Clearance Center Billing and Payment Terms and Conditions, which are incorporated herein by reference.

AIPP or the Copyright Clearance Center may, within two business days of granting this license, revoke the license for any reason whatsoever, with a full refund payable to you. Should you violate the terms of this license at any time, AIPP, AIP Publishing LLC, or Copyright Clearance Center may revoke the license with no refund to you. Notice of such revocation will be made using the contact information provided by you. Failure to receive such notice will not nullify the revocation.

AIPP makes no representations or warranties with respect to the Material. You agree to indemnify and hold harmless AIPP, AIP Publishing LLC, and their officers, directors, employees or agents from and against any and all claims arising out of your use of the Material other than as specifically authorized herein.

The permission granted herein is personal to you and is not transferable or assignable without the prior written permission of AIPP. This license may not be amended except in a writing signed by the party to be charged.

If purchase orders, acknowledgments or check endorsements are issued on any forms containing terms and conditions which are inconsistent with these provisions, such inconsistent terms and conditions shall be of no force and effect. This document, including the CCC Billing and Payment Terms and Conditions, shall be the entire agreement between the parties relating to the subject matter hereof.

This Agreement shall be governed by and construed in accordance with the laws of the State of New York. Both parties hereby submit to the jurisdiction of the courts of New York County for purposes of resolving any disputes that may arise hereunder.

Questions? customercare@copyright.com or +1-855-239-3415 (toll free in the US) or +1-978-646-2777.

Gratis licenses (referencing \$0 in the Total field) are free. Please retain this printable license for your reference. No payment is required. 